\title{
Mathieu's Equation and its Generalizations: Overview of Stability Charts and their Features
}

\author{
Kovacic, Ivana; Rand, Richard H.; Sah, Si Mohamed
}

Published in:

Applied Mechanics Reviews

Link to article, DOI:

10.1115/1.4039144

Publication date:

2018

Document Version

Peer reviewed version

Link back to DTU Orbit

Citation (APA):

Kovacic, I., Rand, R. H., \& Sah, S. M. (2018). Mathieu's Equation and its Generalizations: Overview of Stability Charts and their Features. Applied Mechanics Reviews, 70(2), [020802]. https://doi.org/10.1115/1.4039144

\section{General rights}

Copyright and moral rights for the publications made accessible in the public portal are retained by the authors and/or other copyright owners and it is a condition of accessing publications that users recognise and abide by the legal requirements associated with these rights.

- Users may download and print one copy of any publication from the public portal for the purpose of private study or research.

- You may not further distribute the material or use it for any profit-making activity or commercial gain

- You may freely distribute the URL identifying the publication in the public portal 


\title{
Mathieu's Equation and its Generalizations: Overview of Stability Charts and their Features
}

\author{
Ivana Kovacic ${ }^{1}$, Richard Rand ${ }^{2}$, Si Mohamed Sâh ${ }^{3}$
}

1. Centre of Excellence for Vibro-Acoustic Systems and

Signal Processing, Faculty of Technical Sciences, University of Novi Sad, 21215 Novi Sad, Serbia

2. Department of Mathematics and

Department of Mechanical and Aerospace Engineering, Cornell University, Ithaca NY 14853, USA

3. Technical University of Denmark, Department of Mechanical Engineering, Section of Solid Mechanics, Lyngby, Denmark

\begin{abstract}
This work is concerned with Mathieu's equation - a classical differential equation, which has the form of a linear second-order ordinary differential equation with Cosine-type periodic forcing of the stiffness coefficient, and its different generalisations/extensions. These extensions include: the effects of linear viscous damping, geometric nonlinearity, damping nonlinearity, fractional derivative terms, delay terms, quasiperiodic excitation or elliptic-type excitation. The aim is to provide a systematic overview of the methods to determine the corresponding stability chart, its structure and features, and how it differs from that of the classical Mathieu's equation.
\end{abstract}


Keywords: parametric excitation; stability chart; transition curves; perturbation method; Floquet theory; harmonic balancing; geometric nonlinearity; damping nonlinearity; fractional derivative; delay; quasiperiodic excitation; elliptic-type excitation.

\section{Introduction}

Mathieu's equation is one of the archetypical equations of Nonlinear Vibrations Theory [1]. However, this equation is not only associated with this field, but due to the tools and techniques needed for its quantitative analysis and diverse applications, it appears also in Applied Mathematics [2]-[4] and in many engineering fields [5]-[7].

The form of Mathieu's equation is very simple - it is a linear second-order ordinary differential equation, which differs from the one corresponding to a simple harmonic oscillator in the existence of a time-varying (periodic) forcing of the stiffness coefficient as follows:

$$
\frac{d^{2} x}{d t^{2}}+(\delta+\epsilon \cos t) x=0
$$

where $\delta$ and $\epsilon$ are constant parameters, while $x$ is a dependent variable (its mechanical interpretation will be defined in Section 2.1) and $t$ is time. So, the simple harmonic oscillator is obtained for $\epsilon=0$, and the stiffness parameter $\delta$ corresponds then to the square of its natural frequency, i.e. $\omega_{0}$ $=\sqrt{\delta}$. It is well-known that this oscillator performs free vibrations around the stable equilibrium position $x=0$. However, if the stiffness term contains the parametric excitation, i.e. $\epsilon \neq 0$, the motion can stay bounded (this case is referred to as stable) or the motion becomes unbounded (this case is referred to as unstable). The occurrence of one of these two outcomes depends on the combination of the parameters $\delta$ and $\epsilon$. When presented graphically, this gives the so-called stability chart with regions of stability and regions of instability (tongues) separated by the so-called transition curves, enabling one to clearly determine the resulting behaviour and the stability property mentioned.

Historically speaking, what is now termed 'Mathieu's equation' is attributed to Mathieu's investigations of vibrations in an elliptic drum from 1868 [8]. The extract from this work is presented in Appendix A. It is shown therein how the derivation of Eq. (1) stems from the Helmholtz equation 
for the vibrations of a membrane with an elliptic boundary. Mathieu also developed the power series expansion method, determining the mutual relationships between the stiffness parameter and the amplitude of parametric excitation and the respective solutions of motion. These solutions are called after him 'Mathieu functions' and are presented in Appendix B. The relationships between the stiffness parameter and the amplitude of parametric excitation can be presented graphically (but Mathieu did not do it at that time) and represent the transition curves mentioned above.

A few subsequent important developments of Mathieu's equation are listed below [3]:

1878 Heine expresses the solution as an infinite continued fraction [9].

1883 Floquet presents a generalized treatment of differential equations with periodic coefficients [10].

1886 Hill expresses solution as an infinite determinant [11].

1887 Lord Rayleigh (J.W. Strutt) applies Mathieu's equation to Melde's problem (a tuning fork with an attached string) [12].

1908 Sieger presents the application to diffraction of electromagnetic waves by an elliptic cylinder [13].

1912 Whittaker expresses solution as an integral equation [14].

1915 Ince publishes the first of 18 papers on Mathieu functions, including:

1927 Ince introduces the stability chart [15].

Note that the stability chart is sometimes called 'Strutt diagram' or 'Strutt-Ince diagram'. However, Strutt's work (M.J.O. Strutt, not to be confused with Lord Rayleigh, who is J.W. Strutt) with this chart [16] was published later than Ince's. Thus, it was Ince who first presented it graphically and his figure from [15] is redrawn and included in Appendix B.

The determination and description of the stability chart is the focus of this work. Besides this, the aim is to show how its structure and features change as the form given by Eq. (1) is modified by additional or different geometric, damping and excitation terms. This work is organized as follows. First, a brief overview of mechanical models that are associated with classical Mathieu's equation are given in Section 2. In addition, certain mathematical tools for its quantitative analysis are presented in Section 3, yielding the basic structure and features of the stability chart. Section 4 is concerned with the influence of geometric and damping nonlinearities on this stability chart (it should be noted that Sections 2,3, and 4.1 and Appendix C are strongly based on Rand's online notes [1]). Section 5 deals with the influence of a delay term 
and Section 6 with the effects of a fractional-derivative term. Sections 7 and 8 deal with the effects of different types of excitation: quasiperiodic and elliptic-type excitations.

\section{Classical Mathieu's equation: mechanical models and applications}

Generally speaking and related to nonlinear vibration problems, Mathieu's equation in its classical form (1) is associated with differential equations derived in two general cases [1]: Case 1 - in systems with periodic forcing, and Case 2 - in stability studies of periodic motions in nonlinear autonomous systems.

As illustrative example of Case 1 is a mathematical pendulum whose support moves periodically in a vertical direction (Figure 1a). The governing differential equation is

$$
\frac{d^{2} x}{d t^{2}}+\left(\frac{g}{L}-\frac{A}{L} \cos t\right) \sin x=0,
$$

where $x$ is the generalised coordinate being the angle of deflection, $g$ is the acceleration of gravity, $L$ is the pendulum's length, while the vertical motion of the support is $A \cos t$. Two equilibrium solutions exist: $x=0$ or $x=\pi$. In order to investigate their stability, one would linearize Eq. (2) about the desired equilibrium, deriving an equation of the form of Eq. (1).

If the motion of the support is defined by $A \cos \Omega t$, the equation of motion for small $x$ has the form

$$
\frac{d^{2} x}{d t^{2}}+\left(\omega_{0}^{2}-\frac{A \Omega^{2}}{L} \cos \Omega t\right) x=0,
$$

in which one can recognize two frequencies: the natural frequency $\omega_{0}=\sqrt{g / L}$ and the excitation frequency $\Omega$. However, by introducing $\tau=\Omega t$, one can obtain the form given by Eq. (1) with $\delta=g /\left(L \Omega^{2}\right), \epsilon=-A / L$.

Moreover we can consider the case of the vertically forced inverted pendulum by setting $y=x-\pi$, whereupon the small $y$ differential equation becomes

$$
\frac{d^{2} y}{d t^{2}}-\left(\omega_{0}^{2}-\frac{A \Omega^{2}}{L} \cos \Omega t\right) y=0
$$


Note that in this case the parameter $\delta$ is negative, and hence the unforced equilibrium $y=0$, i.e. $x=\pi$, is unstable. Nevertheless we will show that the equilibrium can be made stable by an appropriate choice of parameter values. This remarkable example was evidently first considered by A. Stephenson in 1908 [17] and [18].

Additional examples that have the same governing equations are, for instance: a frequency-modulated tuned circuit, the Paul trap for charged particles, stability of a floating body, the mirror trap for neutral particles [7], certain autoparametric vibration absorbers [19], stability of elastic systems (bars, for example) under certain time-varying loading [20], asymmetric shaft and bearings in rotor dynamics [21], torsional motions of a rotor in contact with a stator [22], etc. Additional examples from other fields include those from aerospace engineering: for example, helicopter rotor blades in forward flight, attitude stability of satellites in elliptic orbits) and biology (for instance, heart rhythms, membrane vibrations in the inner ear).

As an example of Case 2, one can consider a system called "the particle in the plane" (Figure 1b), which was first studied in [23], [24]. It contains a particle of unit mass which is constrained to move in the $x-y$ plane, and is restrained by two linear springs, each with spring constant $k$ of $1 / 2$. Each of the two springs has unstretched length $L$. The anchor points of the two springs are located on the $x$ axis at $x=1$ and $x=-1$. This autonomous two-degree-of-freedom system has the following equations of motion [23]:

$$
\begin{aligned}
\frac{d^{2} x}{d t^{2}}+(x+1) f_{1}(x, y)+f_{2}(x, y) & =0, \\
\frac{d^{2} y}{d t^{2}}+y f_{1}(x, y)+y f_{2}(x, y) & =0 \\
f_{1}(x, y) & =\frac{1}{2}\left(1-\frac{L}{\sqrt{\left((1+x)^{2}+y^{2}\right)}}\right), \\
f_{2}(x, y) & =\frac{1}{2}\left(1-\frac{L}{\sqrt{\left((1-x)^{2}+y^{2}\right)}}\right) .
\end{aligned}
$$

This system exhibits an exact solution corresponding to a mode of vibration in which the particle moves along the $x$ axis, i.e.:

$$
x=A \cos t, \quad y=0
$$


In order to determine the stability of this motion, one must first derive the equations of motion, then substitute $x=A \cos t+u, y=0+v$, where $u$ and $v$ are small deviations from the motion (9), and then linearize in $u$ and $v$. This results in two linear differential equations on $u$ and $v$. The $u$ equation turns out to be the simple harmonic oscillator, and cannot produce instability. The $v$ equation is [1]:

$$
\frac{d^{2} v}{d t^{2}}+\left(\frac{1-L-A^{2} \cos ^{2} t}{1-A^{2} \cos ^{2} t}\right) v=0
$$

Expanding (10) for small $A$ and setting $\tau=2 t$, one obtains

$$
\frac{d^{2} v}{d \tau^{2}}+\left(\frac{2-2 L-A^{2} L}{8}-\frac{A^{2} L}{8} \cos \tau+O\left(A^{4}\right)\right) v=0,
$$

which is, to $O\left(A^{4}\right)$, in the form of Mathieu's equation (1).

\section{Classical Mathieu's equation: analyses by different approaches}

This Section is divided into two subsections related to two forms of Mathieu's equation: undamped (Section 3.1) and linearly viscously damped (Section 3.2). The first subsection includes the presentation of several quantitative techniques that one can use to solve it: a perturbation method, Floquet theory in conjuction with numerical simulations and harmonic balancing.

\subsection{Undamped case}

\subsubsection{Perturbation method}

In order to find a general solution to Mathieu's equation (1) for small $\epsilon$, one can use the two variable expansion method [1], [25], [26]. Since Eq. (1) is linear, there is no need to stretch time, and one can set $\xi=t$ and $\eta=\epsilon t$, yielding

$$
\frac{\partial^{2} x}{\partial \xi^{2}}+2 \epsilon \frac{\partial^{2} x}{\partial \xi \partial \eta}+\epsilon^{2} \frac{\partial^{2} x}{\partial \eta^{2}}+(\delta+\epsilon \cos \xi) x=0
$$


Next, $x$ is expanded into a power series as follows:

$$
x(\xi, \eta)=x_{0}(\xi, \eta)+\epsilon x_{1}(\xi, \eta)+\cdots .
$$

Substituting Eq. (13) into Eq. (12), neglecting terms of $O\left(\epsilon^{2}\right)$, and collecting terms of the same power of $\epsilon$ gives:

$$
\begin{aligned}
\frac{\partial^{2} x_{0}}{\partial \xi^{2}}+\delta x_{0} & =0 \\
\frac{\partial^{2} x_{1}}{\partial \xi^{2}}+\delta x_{1} & =-2 \frac{\partial^{2} x_{0}}{\partial \xi \partial \eta}-x_{0} \cos \xi
\end{aligned}
$$

Having the form of the equation of motion of a simple harmonic oscillator, the general solution to Eq. (14) is taken in the form:

$$
x_{0}(\xi, \eta)=A(\eta) \cos \sqrt{\delta} \xi+B(\eta) \sin \sqrt{\delta} \xi,
$$

but note that the amplitudes vary with slow time $\eta$. Substituting Eq. (16) into Eq. (15), one derives:

$$
\begin{aligned}
\frac{\partial^{2} x_{1}}{\partial \xi^{2}}+\delta x_{1}= & 2 \sqrt{\delta} \frac{d A}{d \eta} \sin \sqrt{\delta} \xi-2 \sqrt{\delta} \frac{d B}{d \eta} \cos \sqrt{\delta} \xi \\
& -A \cos \sqrt{\delta} \xi \cos \xi-B \sin \sqrt{\delta} \xi \cos \xi
\end{aligned}
$$

Using identities for products of two trig functions, this becomes

$$
\begin{array}{r}
\frac{\partial^{2} x_{1}}{\partial \xi^{2}}+\delta x_{1}= \\
2 \sqrt{\delta} \frac{d A}{d \eta} \sin \sqrt{\delta} \xi-2 \sqrt{\delta} \frac{d B}{d \eta} \cos \sqrt{\delta} \xi \\
-\frac{A}{2}(\cos (\sqrt{\delta}+1) \xi+\cos (\sqrt{\delta}-1) \xi) \\
-\frac{B}{2}(\sin (\sqrt{\delta}+1) \xi+\sin (\sqrt{\delta}-1) \xi) .
\end{array}
$$

For a general value of $\delta$, the first and second term on the right-hand side represent resonance terms and would cause unboundedness of the solution. The removal of resonance terms gives the trivial slow flow:

$$
\frac{d A}{d \eta}=0, \quad \frac{d B}{d \eta}=0
$$


This means that for general $\delta$, the $\cos t$ driving term in Mathieu's equation (1) has no effect. However, if we choose $\delta=\frac{1}{4}$, Eq. (18) becomes

$$
\begin{aligned}
\frac{\partial^{2} x_{1}}{\partial \xi^{2}}+\frac{1}{4} x_{1}= & \frac{d A}{d \eta} \sin \frac{\xi}{2}-\frac{d B}{d \eta} \cos \frac{\xi}{2} \\
& -\frac{A}{2}\left(\cos \frac{3 \xi}{2}+\cos \frac{\xi}{2}\right) \\
& -\frac{B}{2}\left(\sin \frac{3 \xi}{2}-\sin \frac{\xi}{2}\right)
\end{aligned}
$$

Now, there are additional resonance terms and their removal yields the following slow flow:

$$
\frac{d A}{d \eta}=-\frac{B}{2}, \quad \frac{d B}{d \eta}=-\frac{A}{2} \quad \Rightarrow \quad \frac{d^{2} A}{d \eta^{2}}=\frac{A}{4}
$$

It can be concluded that $A(\eta)$ and $B(\eta)$ grow exponentially, and, thus, the parameter value $\delta=\frac{1}{4}$ causes instability. This corresponds to a 2:1 subharmonic resonance in which the driving frequency is twice the natural frequency.

This may be generalized by "detuning" the resonance, that is, by expanding $\delta$ in a power series in $\epsilon[1]$ :

$$
\delta=\frac{1}{4}+\delta_{1} \epsilon+\delta_{2} \epsilon^{2}+\cdots
$$

Now Eq. (15) gets an additional term:

$$
\frac{\partial^{2} x_{1}}{\partial \xi^{2}}+\frac{1}{4} x_{1}=-2 \frac{\partial^{2} x_{0}}{\partial \xi \partial \eta}-x_{0} \cos \xi-\delta_{1} x_{0}
$$

which results in the following additional terms in the slow flow Eqs. (21):

$$
\frac{d A}{d \eta}=\left(\delta_{1}-\frac{1}{2}\right) B, \quad \frac{d B}{d \eta}=-\left(\delta_{1}+\frac{1}{2}\right) A \quad \Rightarrow \quad \frac{d^{2} A}{d \eta^{2}}+\left(\delta_{1}^{2}-\frac{1}{4}\right) A=0 .
$$

It is seen now that $A(\eta)$ and $B(\eta)$ will be sine and cosine functions of slow time $\eta$ if $\delta_{1}^{2}-\frac{1}{4}>0$, that is, if either $\delta_{1}>\frac{1}{2}$ or $\delta_{1}<-\frac{1}{2}$. Thus, the 
following two curves in the $\delta-\epsilon$ plane represent stability changes, and are called transition curves as noted in the Introduction:

$$
\delta=\frac{1}{4} \pm \frac{\epsilon}{2}+O\left(\epsilon^{2}\right) .
$$

These two transition curves emanate from the point $\delta=\frac{1}{4}$ on the $\delta$ axis and define a region of instability called a tongue (Figure 2a) as also noted in the Introduction. Inside the tongue, for small $\epsilon, x$ grows in time (Figure 2b), so this region is labelled by $\mathrm{U}$ (unstable). Equations (16) and (24) imply that outside the tongue (but not in or near another resonance tongue), $x$ is the sum of terms each of which is the product of two periodic (sinusoidal) functions with generally incommensurate frequencies, that is, $x$ is a quasiperiodic function of $t$ (Figure 2c) and this region is labelled by $\mathrm{S}$ (stable).

\subsubsection{Floquet theory and numerical approach}

This section is concerned with the application of Floquet theory - the general theory of linear differential equations with periodic coefficients, to Mathieu's equation (1) [1]. When using perturbation analysis, the parameter $\epsilon$ is assumed to be small $(\epsilon<<1)$, while this assumption is not required and large values of epsilon can be used in this approach.

First, Mathieu's equation (1) is represented in a generalization form, called Hill's equation:

$$
\frac{d^{2} x}{d t^{2}}+f(t) x=0, \quad f(t+T)=f(t)
$$

Here $x$ and $f$ are scalars, and $f(t)$ represents a general periodic function with period $T$. Thus, in the case of Eq. (1), one has $f(t)=\delta+\epsilon \cos t$ and $T=2 \pi$.

By defining $x_{1}=x$ and $x_{2}=\frac{d x}{d t}$, Eq. (26) can be written as a system of two first order ordinary differential equations:

$$
\frac{d}{d t}\left[\begin{array}{l}
x_{1} \\
x_{2}
\end{array}\right]=\left[\begin{array}{cc}
0 & 1 \\
-f(t) & 0
\end{array}\right]\left[\begin{array}{l}
x_{1} \\
x_{2}
\end{array}\right]
$$

Next, a fundamental solution matrix out of two solution vectors, $\left[\begin{array}{l}x_{11}(t) \\ x_{12}(t)\end{array}\right]$ and $\left[\begin{array}{l}x_{21}(t) \\ x_{22}(t)\end{array}\right]$ is constructed, satisfying the initial conditions:

$$
\left[\begin{array}{l}
x_{11}(0) \\
x_{12}(0)
\end{array}\right]=\left[\begin{array}{l}
1 \\
0
\end{array}\right], \quad\left[\begin{array}{l}
x_{21}(0) \\
x_{22}(0)
\end{array}\right]=\left[\begin{array}{l}
0 \\
1
\end{array}\right]
$$


The matrix $C$ is the evaluation of the fundamental solution matrix at time $T$ :

$$
C=\left[\begin{array}{ll}
x_{11}(T) & x_{21}(T) \\
x_{12}(T) & x_{22}(T)
\end{array}\right]
$$

From Floquet theory [1], it is known that stability is determined by the eigenvalues (characteristic multipliers) of $C$ :

$$
\lambda^{2}-(\operatorname{tr} C) \lambda+\operatorname{det} C=0,
$$

where $\operatorname{tr} C$ and $\operatorname{det} C$ are the trace and determinant of $C$. Now Eq. (26) has the special property that $\operatorname{det} C=1$. This may be shown by defining $W$ (the Wronskian) as:

$$
W(t)=\operatorname{det} C=x_{11}(t) x_{22}(t)-x_{12}(t) x_{21}(t) .
$$

Taking the time derivative of $W$ and using Eq. (27) gives that $\frac{d W}{d t}=0$, which implies that $W(t)=$ constant $=W(0)=1$. Thus, Eq. (30) can be written down as:

$$
\lambda^{2}-(\operatorname{tr} C) \lambda+1=0 .
$$

Its solutions are:

$$
\lambda_{1,2}=\frac{\operatorname{tr} C \pm \sqrt{\operatorname{tr} C^{2}-4}}{2} .
$$

Floquet theory [1] showed that instability results if either eigenvalue has modulus larger than unity. Thus, if $|\operatorname{tr} C|>2$, then Eq. (33) gives real roots. But the product of the roots is unity, so if one root has modulus less than unity, the other has modulus greater than unity, with the result that this case is unstable and corresponds to exponential growth in time. On the other hand, if $|\operatorname{tr} C|<2$, then Eq. (33) gives a pair of complex conjugate roots. But since their product must be unity, they must both lie on the unit circle, with the result that this case is stable. Note that the stability here is neutral stability not asymptotic stability, since Eq. (26) has no damping. This case corresponds to quasiperiodic behavior in time.

So, the transition from stable to unstable corresponds to those parameter values which give $|\operatorname{tr} C|=2$. From Eq. (33), if $\operatorname{tr} C=2$ then $\lambda_{1,2}=1,1$ and this corresponds to a periodic solution with period $T$. On the other hand, if $\operatorname{tr} C=-2$ then $\lambda_{1,2}=-1,-1$. This corresponds to a periodic solution with 
period $2 T$. This analysis gives the important result that on the transition curves in parameter space between stable and unstable, there exist periodic motions of period $T$ or $2 T$.

The theory presented in this section can be used as a practical numerical procedure for determining stability of Eq. (26). One needs to begin by numerically integrating the ordinary differential equation for the two initial conditions (28); carry each numerical integration out to time $t=T$ and so obtain $\operatorname{tr} C=x_{11}(T)+x_{22}(T)$; then, $|\operatorname{tr} C|>2$ is unstable, while $|\operatorname{tr} C|<2$ is stable. Note that this approach allows one to draw conclusions about large time behavior after numerically integrating for only one forcing period. Without Floquet theory, one would have to numerically integrate out to large time in order to determine if a solution was growing unbounded, especially for systems which are close to a transition curve, in which case the asymptotic growth is very slow.

A stability chart of Mathieu's equation with several tongues obtained by using numerical integration in conjunction with Floquet theory is shown in Figure 3. Note that there are stable regions in the negative half-plane $\delta<0$. By choosing parameters so that the system lies in one of these stable regions for negative $\delta$, we may stabilize an equilibrium which is unstable in the unforced system. An example is the periodically forced inverted pendulum discussed in Eq. (4).

\subsubsection{Harmonic balancing}

The transition curves (25) found earlier in Section 3.1.1 cover the first tongue only. The question that naturally arises is [1]: Why did the perturbation method miss the other tongues of instability? It was because the perturbation method was truncated, neglecting terms of $O\left(\epsilon^{2}\right)$. The other tongues of instability turn out to emerge at higher order truncations in the various perturbation methods (two variable expansion, averaging, Lie transforms, normal forms, even regular perturbations). In all cases these methods deliver an expression for a particular transition curve in the form of a power series expansion:

$$
\delta=\frac{n^{2}}{4}+\delta_{1} \epsilon+\delta_{2} \epsilon^{2}+\cdots
$$


Since the period of the forcing function in Eq. (1) is $T=2 \pi$, one may apply the result obtained in the previous section to conclude that on the transition curves in the $\delta-\epsilon$ parameter plane there exist solutions of period $2 \pi$ or $4 \pi$. This motivates us to look for such a solution in the form of a Fourier series [1]:

$$
x(t)=\sum_{n=0}^{\infty} a_{n} \cos \frac{n t}{2}+b_{n} \sin \frac{n t}{2} .
$$

This series represents a general periodic function with period $4 \pi$, and includes functions with period $2 \pi$ as a special case (when $a_{\text {odd }}$ and $b_{\text {odd }}$ are zero). Substituting Eq. (35) into Mathieu's equation (1), simplifying the trig and collecting terms with the same angular frequency (a procedure called harmonic balancing), gives four sets of algebraic equations on the coefficients $a_{\text {even }}, b_{\text {even }}, a_{\text {odd }}$ and $b_{\text {odd }}$. Each set is homogeneous and of infinite order, so for a nontrivial solution the determinants must vanish. This gives four infinite determinants (called Hill's determinants) [1]:

$$
\begin{aligned}
& a_{\text {even }}: \quad\left|\begin{array}{ccccc}
\delta & \epsilon / 2 & 0 & 0 & \\
\epsilon & \delta-1 & \epsilon / 2 & 0 & \cdots \\
0 & \epsilon / 2 & \delta-4 & \epsilon / 2 & \\
& & \cdots &
\end{array}\right|=0 \\
& b_{\text {even }}: \quad\left|\begin{array}{ccccc}
\delta-1 & \epsilon / 2 & 0 & 0 & \\
\epsilon / 2 & \delta-4 & \epsilon / 2 & 0 & \cdots \\
0 & \epsilon / 2 & \delta-9 & \epsilon / 2 & \\
& & \cdots &
\end{array}\right|=0 \\
& a_{\text {odd }}: \quad\left|\begin{array}{ccccc}
\delta-1 / 4+\epsilon / 2 & \epsilon / 2 & 0 & 0 & \\
\epsilon / 2 & \delta-9 / 4 & \epsilon / 2 & 0 & \ldots \\
0 & \epsilon / 2 & \delta-25 / 4 & \epsilon / 2 & \\
0 & & \ldots & &
\end{array}\right|=0 \text {, } \\
& b_{\text {odd }}: \quad \begin{array}{ccccc}
\delta-1 / 4-\epsilon / 2 & \epsilon / 2 & 0 & 0 & \\
\epsilon / 2 & \delta-9 / 4 & \epsilon / 2 & 0 & \cdots \\
0 & \epsilon / 2 & \delta-25 / 4 & \epsilon / 2 & \\
& & \cdots &
\end{array} \mid=0 .
\end{aligned}
$$


In all four determinants the typical row is of the form:

$$
\cdots \quad 0 \quad \epsilon / 2 \quad \delta-n^{2} / 4 \quad \epsilon / 2 \quad 0 \quad \cdots,
$$

(except for the first one or two rows).

Each of these four determinants represents a functional relationship between $\delta$ and $\epsilon$, which plots as a set of transition curves in the $\delta$ - $\epsilon$ plane. By setting $\epsilon=0$ in these determinants it is easy to see where the associated curves intersect the $\delta$ axis. The transition curves obtained from the $a_{\text {even }}$ and $b_{\text {even }}$ determinants intersect the $\delta$ axis at $\delta=n^{2}, n=0,1,2, \cdots$, while those obtained from the $a_{\text {odd }}$ and $b_{\text {odd }}$ determinants intersect the $\delta$ axis at $\delta=\frac{(2 n+1)^{2}}{4}, n=0,1,2, \cdots$. For $\epsilon>0$, each of these points on the $\delta$ axis gives rise to two transition curves, one coming from the associated $a$ determinant, and the other from the $b$ determinant. Thus, there is a tongue of instability emanating from each of the following points on the $\delta$ axis:

$$
\delta=\frac{n^{2}}{4}, \quad n=0,1,2,3, \cdots .
$$

The $n=0$ case is an exception as only one transition curve emanates from it, as a comparison of Eq. (36) with Eq. (37) will show.

Let us now substitute Eq. (34) for $n=1$ into the $a_{\text {odd }}$ determinant (38). Expanding a $3 \times 3$ truncation of Eq. (38), one can get (using computer algebra):

$$
-\frac{\epsilon^{3}}{8}-\frac{\delta \epsilon^{2}}{2}+\frac{13 \epsilon^{2}}{8}+\frac{\delta^{2} \epsilon}{2}-\frac{17 \delta \epsilon}{4}+\frac{225 \epsilon}{32}+\delta^{3}-\frac{35 \delta^{2}}{4}+\frac{259 \delta}{16}-\frac{225}{64}=0 .
$$

Substituting Eq. (34) with $n=1$ into Eq. (42) and collecting terms gives:

$$
\left(12 \delta_{1}+6\right) \epsilon+\frac{\left(24 \delta_{2}-16 \delta_{1}^{2}-8 \delta_{1}+3\right) \epsilon^{2}}{2}+\cdots .
$$

Requiring the coefficients of $\epsilon$ and $\epsilon^{2}$ in Eq. (43) to vanish gives:

$$
\delta_{1}=-\frac{1}{2}, \quad \delta_{2}=-\frac{1}{8} .
$$

This process can be continued to any order of truncation. The expansions of the first nine transition curves obtained in this way are given in Appendix 
C. The transition curves are also plotted in Figure 3 as the red thick lines and clearly match the numerical solution for the values of $\epsilon$ shown.

Note that the procedure presented above is concerned with Eq. (1). However, if one would rather stick to the equation of motion that contains the natural and excitation frequency as in Eq. (3), the procedure presented can be generalized by relating $\omega_{0}$ to $n \Omega / 2$ (i.e. $\omega_{0}^{2}$ to $\left.(n \Omega / 2)^{2}\right)$ starting from Eq. (1) instead of relating $\delta$ to $n^{2} / 4$.

\subsection{The case with linear viscous damping}

In this section we investigate the effect that damping has on the transition curves of Mathieu's equation by applying the two variable expansion method to the following equation, known as the damped Mathieu equation [1]:

$$
\frac{d^{2} x}{d t^{2}}+c \frac{d x}{d t}+(\delta+\epsilon \cos t) x=0
$$

In order to apply the perturbation method, the damping coefficient $c$ is also rescaled to be $O(\epsilon)$ :

$$
c=\epsilon \mu \text {. }
$$

The same algorithm used earlier in Section 3.1.1, is also utilized here so that Eq. (45) becomes:

$$
\frac{\partial^{2} x}{\partial \xi^{2}}+2 \epsilon \frac{\partial^{2} x}{\partial \xi \partial \eta}+\epsilon^{2} \frac{\partial^{2} x}{\partial \eta^{2}}+\epsilon \mu\left(\frac{\partial x}{\partial \xi}+\epsilon \frac{\partial x}{\partial \eta}\right)+(\delta+\epsilon \cos \xi) x=0 .
$$

Now, $x$ is expanded as in Eq. (13) and $\delta$ as in Eq. (22). As a result, Eq. (23) gets an additional term:

$$
\frac{\partial^{2} x_{1}}{\partial \xi^{2}}+\frac{1}{4} x_{1}=-2 \frac{\partial^{2} x_{0}}{\partial \xi \partial \eta}-x_{0} \cos \xi-\delta_{1} x_{0}-\mu \frac{\partial x_{0}}{\partial \xi}
$$

which results in two additional terms appearing in the slow flow Eqs. (24):

$$
\frac{d A}{d \eta}=-\frac{\mu}{2} A+\left(\delta_{1}-\frac{1}{2}\right) B, \quad \frac{d B}{d \eta}=-\left(\delta_{1}+\frac{1}{2}\right) A-\frac{\mu}{2} B .
$$

Equations (49) represent a linear constant coefficient system which may be solved by assuming a solution in the form $A(\eta)=A_{0} \exp (\lambda \eta), B(\eta)=$ 
$B_{0} \exp (\lambda \eta)$. For nontrivial constants $A_{0}$ and $B_{0}$, the following determinant must vanish:

$$
\left|\begin{array}{cc}
-\frac{\mu}{2}-\lambda & -\frac{1}{2}+\delta_{1} \\
-\frac{1}{2}-\delta_{1} & -\frac{\mu}{2}-\lambda
\end{array}\right|=0 \quad \Rightarrow \quad \lambda=-\frac{\mu}{2} \pm \sqrt{-\delta_{1}^{2}+\frac{1}{4}} .
$$

For the transition between stable and unstable, one should set $\lambda=0$, deriving the following value for $\delta_{1}$ :

$$
\delta_{1}= \pm \frac{\sqrt{1-\mu^{2}}}{2}
$$

This gives the following expressions for the $n=1$ transition curves:

$$
\delta=\frac{1}{4} \pm \epsilon \frac{\sqrt{1-\mu^{2}}}{2}+O\left(\epsilon^{2}\right)=\frac{1}{4} \pm \frac{\sqrt{\epsilon^{2}-c^{2}}}{2}+O\left(\epsilon^{2}\right) .
$$

Equation (52) predicts that for a given value of $c$ there is a minimum value of $\epsilon$ which is required for instability to occur. The $n=1$ tongue, which for $c=0$ emanates from the $\delta$ axis, becomes detached from the $\delta$ axis for $c>0$. Figure 4a contains the transition curves (52) for the first tongue both for the undamped and damped case - it is clearly seen how the tongue shifts up due to the existence of linear viscous damping. In order to determine the transition curves for other regions, one can use the same approach, but substitute Eq. (34) into Eq. (47).

The stability chart of the damped Mathieu equation (45) is obtained numerically based on Floquet theory and presented in Figure 4b. It enables one to compare it with the undamped case for several instability tongues. Note that in this case, one of the characteristic multipliers is always located within the unit circle and both characteristic multipliers can never cross the unit circle at the same time. The system can be asymptotically stable with $\left|\lambda_{2}\right| \leq\left|\lambda_{1}\right|<1$. Thus, in the damped Mathieu equation (45), cyclic-fold or period-doubling bifurcations may occur. The transition curves of the odd regions (first, second) are associated with period-doubling bifurcations, while the transition curves of the even regions are related to cyclic-fold bifurcations $[27]$. 


\section{Mathieu's equation with nonlinearities}

\subsection{Geometric nonlinearity}

It is shown in Figure 2 that an unbounded solution to Mathieu's equation (1) can result from resonances between the forcing frequency and the oscillator's unforced natural frequency. However, nonlinear systems do not exhibit unbounded behavior. The difference lies in the fact that Mathieu's equation is linear. The effects of nonlinearity can be explained as follows: as the resonance causes the amplitude of the motion to increase, the relation between period and amplitude (which is a characteristic effect of nonlinearity - see, for example, [28]) causes the resonance to detune, decreasing its tendency to produce large motions.

A more realistic model can be obtained by including nonlinear terms in Mathieu's equation. For example, in the case of the vertically driven pendulum (Figure 1a), after expanding $\sin x$ from Eq. (2) in a Taylor series, one can derive [1]:

$$
\frac{d^{2} x}{d t^{2}}+\left(\frac{g}{L}-\frac{A \omega^{2}}{L} \cos \omega t\right)\left(x-\frac{x^{3}}{6}+\cdots\right)=0 .
$$

Now if we rescale time by $\tau=\omega t$ and set $\delta=\frac{g}{\omega^{2} L}$ and $\epsilon=\frac{A}{L}$, we get:

$$
\frac{d^{2} x}{d \tau^{2}}+(\delta-\epsilon \cos \tau)\left(x-\frac{x^{3}}{6}+\cdots\right)=0 .
$$

Next, if we scale $x$ by $x=\sqrt{\epsilon} y$ and neglect terms of $O\left(\epsilon^{2}\right)$, we obtain:

$$
\frac{d^{2} y}{d \tau^{2}}+(\delta-\epsilon \cos \tau) y-\epsilon \frac{\delta}{6} y^{3}+O\left(\epsilon^{2}\right)=0 .
$$

Motivated by this example, in this section we study the following nonlinear Mathieu equation:

$$
\frac{d^{2} x}{d t^{2}}+(\delta+\epsilon \cos t) x+\epsilon \alpha x^{3}=0 .
$$

This can be referred to as the Mathieu-Duffing equation as it contains a cubic nonlinear term, which is associated with the Duffing equation [29]. 
One can once again use the two variable expansion method to treat this equation. Using the same setup that we did earlier in Section 3, Eq. (56) becomes:

$$
\frac{\partial^{2} x}{\partial \xi^{2}}+2 \epsilon \frac{\partial^{2} x}{\partial \xi \partial \eta}+\epsilon^{2} \frac{\partial^{2} x}{\partial \eta^{2}}+(\delta+\epsilon \cos \xi) x+\epsilon \alpha x^{3}=0
$$

Expanding $x$ as in Eq. (13) and $\delta$ as in Eq. (22), and one finds that Eq. (23) gets an additional term:

$$
\frac{\partial^{2} x_{1}}{\partial \xi^{2}}+\frac{1}{4} x_{1}=-2 \frac{\partial^{2} x_{0}}{\partial \xi \partial \eta}-x_{0} \cos \xi-\delta_{1} x_{0}-\alpha x_{0}^{3}
$$

where $x_{0}$ is of the form:

$$
x_{0}(\xi, \eta)=A(\eta) \cos \frac{\xi}{2}+B(\eta) \sin \frac{\xi}{2} .
$$

Removal of resonant terms in Eq. (58) results in the appearance of some additional cubic terms in the slow flow Eqs. (24):

$$
\frac{d A}{d \eta}=\left(\delta_{1}-\frac{1}{2}\right) B+\frac{3 \alpha}{4} B\left(A^{2}+B^{2}\right), \quad \frac{d B}{d \eta}=-\left(\delta_{1}+\frac{1}{2}\right) A-\frac{3 \alpha}{4} A\left(A^{2}+B^{2}\right) .
$$

In order to solve these equations, one can turn to polar coordinates in the $A-B$ phase plane, introducing:

$$
A=R \cos \theta, \quad B=R \sin \theta
$$

Equations (61) and (59) give now the following alternate expression for $x_{0}$ :

$$
x_{0}(\xi, \eta)=R(\eta) \cos \left(\frac{\xi}{2}-\theta(\eta)\right) .
$$

Substituting Eq. (61) into the slow flow (60) gives:

$$
\frac{d R}{d \eta}=-\frac{R}{2} \sin 2 \theta, \quad \frac{d \theta}{d \eta}=-\delta_{1}-\frac{\cos 2 \theta}{2}-\frac{3 \alpha}{4} R^{2} .
$$

Equilibria of the slow flow (63) are considered now. From Eq. (62), a solution in which $R$ and $\theta$ are constant in slow time $\eta$ represents a periodic motion of the nonlinear Mathieu equation (56) which has one-half the frequency of the 
forcing function, that is, such a motion is a 2:1 subharmonic motion. Such slow flow equilibria satisfy the equations:

$$
-\frac{R}{2} \sin 2 \theta=0, \quad-\delta_{1}-\frac{\cos 2 \theta}{2}-\frac{3 \alpha}{4} R^{2}=0 .
$$

Ignoring the trivial solution $R=0$, the first Eq. of (64) requires $\sin 2 \theta=0$ or $\theta=0, \frac{\pi}{2}, \pi$ or $\frac{3 \pi}{2}$. Solving the second Eq. of (64) for $R^{2}$, one can get:

$$
R^{2}=-\frac{4}{3 \alpha}\left(\frac{\cos 2 \theta}{2}+\delta_{1}\right) .
$$

For a nontrivial real solution one requires that $R^{2}>0$. Let us assume that the nonlinearity parameter $\alpha>0$, which corresponds to the case of hardening Duffing nonlinearity [29]. Then in the case of $\theta=0$ or $\pi, \cos 2 \theta=1$ and nontrivial equilibria exist only for $\delta_{1}<-\frac{1}{2}$. On the other hand, for $\theta=\frac{\pi}{2}$ or $\frac{3 \pi}{2}, \cos 2 \theta=-1$ and nontrivial equilibria require $\delta_{1}<\frac{1}{2}$. These cases with different equilibria are illustrated in Figure 5a. Since $\delta_{1}= \pm \frac{1}{2}$ corresponds to transition curves for the stability of the trivial solution, the analysis predicts that bifurcations occur as one crosses the transition curves in the $\delta$ - $\epsilon$ plane. That is, imagine quasistatically decreasing the parameter $\delta$ while $\epsilon$ is kept fixed, and moving through the $n=1$ tongue emanating from the point $\delta=\frac{1}{4}$ on the $\delta$ axis. As $\delta$ decreases across the right transition curve, the trivial solution $x=0$ becomes unstable and simultaneously a stable 2:1 subharmonic motion is born. This motion grows in amplitude as $\delta$ continues to decrease. When the left transition curve is crossed, the trivial solution becomes stable again, and an unstable 2:1 subharmonic is born. This scenario can be pictured as involving two pitchfork bifurcations, which is illustrated in Figure 5b.

If the nonlinearity parameter $\alpha<0$, a similar sequence of bifurcations occurs, except in this case the subharmonic motions are born as $\delta$ increases quasistatically through the $n=1$ tongue. 


\subsection{Damping nonlinearity}

This section is concerned with quadratically damped Mathieu's equation:

$$
\frac{d^{2} x}{d t^{2}}+\frac{d x}{d t}\left|\frac{d x}{d t}\right|+(\delta+\epsilon \cos t) x=0 .
$$

This equation of motion governs the dynamics of a cable towed by a submarine, and its derivation and physical interpretations are given in [30].

Equation (66) admits the exact solution $x=0$, and its stability is governed by the classical Mathieu equation (1). Although the linear theory predicts that inside its tongues the motion will be unbounded, the existence of the nonlinear term in Eq. (66) causes the resonance to detune as the amplitude of $x$ grows. As a result, a periodic motion having finite amplitude is created inside the tongues, at least for small $\epsilon$.

So, for small values of the parameter $\epsilon$, the method of averaging can be used to obtain approximate expressions for periodic solutions to the nonlinear equation (66). Inside the first tongue, a detuning parameter is introduced as follows

$$
\delta=\frac{1}{4}+\epsilon \delta_{1}
$$

and a solution is assumed in the form

$$
x(t)=R \cos \left(\frac{t}{2}+\psi\right),
$$

where $R$ and $\psi$ are slowly varying in time $t$. The method of averaging gives the following expressions for them, and this corresponds to stable periodic motion:

$$
R=\frac{3 \pi}{4} \sqrt{1-4 \delta_{1}^{2}}, \quad \psi=\frac{1}{2} \cos ^{-1}\left(-2 \delta_{1}\right) .
$$

This result states that there is an attractive period-2 subharmonic motion inside the first tongue for small $\epsilon$.

Let us not carry out this analysis for the second tongue. In this case, it is necessary to include terms of order $\epsilon^{2}$ and the detuning takes the form

$$
\delta=1+\epsilon \delta_{1}+\varepsilon^{2} \delta_{2}
$$

Equation (66) becomes 


$$
\begin{aligned}
\ddot{x}+x & =-\epsilon G_{1}-\epsilon^{2} G_{2}, \\
G_{1} & =\delta_{1} x+x \cos t+\dot{x}^{2} \operatorname{sgn}(\dot{x}), \\
G_{2} & =\delta_{2} x .
\end{aligned}
$$

For $\epsilon=0$, Eq. (71) has the solution

$$
\begin{aligned}
& x(t)=R \cos (t+\psi), \\
& \dot{x}(t)=-R \sin (t+\psi) .
\end{aligned}
$$

This solution is the basis for the variation of parameters, which yields to the following slow flow equations

$$
\begin{aligned}
\dot{R} & =\epsilon G_{1} \sin (t+\psi)+\epsilon^{2} G_{2} \sin (t+\psi), \\
R \dot{\psi} & =\epsilon G_{1} \cos (t+\psi)+\epsilon^{2} G_{2} \cos (t+\psi) .
\end{aligned}
$$

Further, a near identity transformation is introduced in the form

$$
\begin{aligned}
& R=a+\epsilon W_{1}(a, \psi, t)+\epsilon^{2} V_{1}(a, \psi, t)+\ldots \\
& \psi=\psi+\epsilon W_{2}(a, \psi, t)+\epsilon^{2} V_{2}(a, \psi, t)+\ldots
\end{aligned}
$$

where $W_{1}, W_{2}, V_{1}$ and $V_{2}$ are called generating functions chosen to make the transformed equations on $a$ and new $\psi$ as simple as possible.

In order to deal with the signum function in Eq. (72), it is expanded into a Fourier sine series

$$
\operatorname{sgn}(\sin (t+\psi))=\frac{4}{\pi} \sum_{n=1}^{\infty} \frac{1}{2 n-1} \sin [(2 n-1)(t+\psi)] .
$$

Carrying out the averaging, the slow equations are found to be

$$
\begin{aligned}
& \dot{a}=-\epsilon \frac{4 a^{2}}{3 \pi}+\epsilon^{2}\left(-\frac{a}{8} \sin 2 \psi-\frac{a^{2} \delta_{1}}{3 \pi}\right) \\
& \dot{\psi}=\epsilon \frac{\delta_{1}}{2}+\epsilon^{2}\left(-\frac{1}{8} \cos 2 \psi+\frac{\delta_{2}}{2}-\frac{\delta_{1}^{2}}{8}-\frac{Q a^{2}}{\pi^{2}}-\frac{1}{12}\right),
\end{aligned}
$$


where $Q$ is a constant that depends on the order of truncation of the Fourier series. Numerical results indicate that $Q$ converges to a value near 1.05261. However, its precise value is not of importance as it appears in a higher order term in the slow flow equations. Based on the previous equations, the following fixed points are obtained:

$$
\begin{aligned}
a & =-\epsilon \frac{\pi}{32} \sqrt{5+48 \delta_{2}-144 \delta_{2}^{2}}, \\
\psi & =\frac{1}{2} \cos ^{-1}\left[\frac{2}{3}\left(6 \delta_{2}-1\right)\right] .
\end{aligned}
$$

These expressions describe two fixed points. Unlike in the first order averaging near the first tongue, the amplitude of the limit cycles in this second tongue depend on the value of the parameter $\epsilon$.

In [31], numerical investigations of the quadratically damped Mathieu equation (66) are accomplished by generating a Poincaré map corresponding to a surface of section $t=0 \bmod 2 \pi$. A variety of periodic motions are observed, depending upon the location in the $\delta-\epsilon$ parameter plane. Figure 6 shows schematically the different Poincaré map portraits that are exhibited by Eq. (66). Outside the instability regions, the origin is always stable, as indicated by a lone spiral to the origin. Inside the instability regions, the origin is unstable, as indicated by a saddle-like $x$ at the origin. Inside the $2: 1$ region the two spiral singularities in the Poincaré map represent a single period $4 \pi$ motion, whereas in the $1: 1$ region they represent two period $2 \pi$ motions. As the 2:1 region is exited above point $\mathrm{P}$ into the region marked $\mathrm{R} 1$, a subcritical pitchfork bifurcation occurs. In this case, the origin becomes stable and an unstable 2:1 subharmonic periodic motion is created. As region $\mathrm{R} 1$ is exited into region $\mathrm{R} 2$, the $1: 1$ transition curve is crossed, and the expected supercritical pitchfork bifurcation curve takes place at the origin. The origin once more becomes unstable, while two stable period $2 \pi$ motions are born out of the origin. The most interesting feature displayed in Figure 6 corresponds to what happens when one moves from either of regions R1 or R2 downward across the nearly-straight line bifurcation curve emanating from point $\mathrm{P}$. In this case the two coexisting outermost periodic orbits - the stable and unstable period $4 \pi$ orbits - coalesce and are destroyed in a saddlenode bifurcation. It is seen that this saddle-node bifurcation does not take place at the origin. An analytic approximation for this curve on which this secondary bifurcation takes place is obtained in [31]. This involved perturb- 
ing directly off of Mathieu's equation and using Mathieu functions instead of the usual sines and cosines. An interesting feature of this method is its semi-analytical nature. Because Mathieu functions do not have closed-form representations that are easy to manipulate, the method needed to be executed semi-analytically, that is, certain integrals had to be evaluated by numerical quadrature. When combined with Padé approximants, the perturbation method recovered an acceptable approximation to the secondary bifurcation curve in a neighborhood of point $\mathrm{P}$.

\section{Mathieu's equation with delay}

In this section we consider a delayed Mathieu equation. Adding a delay to a differential equation increases the dimensionality of the system, which makes the investigations of delay differential equations (DDEs) with analytical methods challenging. In a DDE, the state variables depend not only on the present time but also on a previous time. In this section we first find the stability charts of the delayed linear Mathieu equation using the two variable method, and then we consider Hopf bifurcation in a delayed nonlinear Mathieu equation.

\subsection{Delayed linear Mathieu's equation}

The delayed linear Mathieu equation under consideration is given by

$$
\ddot{x}+\epsilon \mu \dot{x}+(\delta+\epsilon \alpha \cos t) x=b x(t-\tau),
$$

where $\tau$ is delay and $b=\epsilon \beta$ is the corresponding constant coefficient in front of the delayed term.

The objective here is to determine the stability chart that corresponds to the 2:1 parametric resonance, which emanates from $\delta=1 / 4$. Hence, this value is perturbed off as follows:

$$
\delta=\frac{1}{4}+\epsilon \delta_{1} .
$$

The two variable expansion method is to be used further. The solution is, thus, assumed to depend on two time variables: $x(\xi, \eta)$, where $\xi=t$ and $\eta=\epsilon t$. Then, one has

$$
x_{d}=x(t-\tau)=x(\xi-\tau, \eta-\epsilon \tau) .
$$


Dropping terms of $O\left(\epsilon^{2}\right)$, Eq. (85) becomes

$$
\frac{\partial^{2} x}{\partial \xi^{2}}+2 \epsilon \frac{\partial^{2} x}{\partial \xi \partial \eta}+\epsilon \mu \frac{\partial x}{\partial \xi}+(\delta+\epsilon \alpha \cos \xi) x=\epsilon \beta x(\xi-\tau, \eta-\epsilon \tau)
$$

Expanding $x$ in a power series in $\epsilon, x=x_{0}+\epsilon x_{1}+O\left(\epsilon^{2}\right)$, and collecting terms, one can obtain

$$
\begin{gathered}
\frac{\partial^{2} x_{0}}{\partial \xi^{2}}+\frac{1}{4} x_{0}=0 \\
\frac{\partial^{2} x_{1}}{\partial \xi^{2}}+\frac{1}{4} x_{1}=-2 \frac{\partial^{2} x_{0}}{\partial \xi \partial \eta}-\mu \frac{\partial x_{0}}{\partial \xi}-\delta_{1} x_{0}-\alpha x_{0} \cos \xi+\beta x_{0}(\xi-\tau, \eta-\epsilon \tau)
\end{gathered}
$$

The solution of Eq. (89) can be expressed as:

$$
x_{0}(\xi, \eta)=A(\eta) \cos \frac{\xi}{2}+B(\eta) \sin \frac{\xi}{2} .
$$

Equation (90) requires the term $x_{0}(\xi-\tau, \eta-\epsilon \tau)$ to be determined:

$$
x_{0}(\xi-\tau, \eta-\epsilon \tau)=A_{d} \cos \frac{(\xi-\tau)}{2}+B_{d} \sin \frac{(\xi-\tau)}{2},
$$

where $A_{d}=A(\eta-\epsilon \tau)$ and $B_{d}=B(\eta-\epsilon \tau)$.

Substituting Eq. (91) and (92) into Eq. (90) and eliminating resonant terms, the following is derived for the slow flow:

$$
\begin{aligned}
\frac{d A}{d \eta} & =-\frac{\mu}{2} A+\left[-\frac{\alpha}{2}+\delta_{1}\right] B-\beta A_{d} \sin \frac{\tau}{2}-\beta B_{d} \cos \frac{\tau}{2} \\
\frac{d B}{d \eta} & =\left[-\frac{\alpha}{2}-\delta_{1}\right] A-\frac{\mu}{2} B+\beta A_{d} \cos \frac{\tau}{2}-\beta B_{d} \sin \frac{\tau}{2}
\end{aligned}
$$

Equations (93), (94) are a linear constant coefficient system which may be solved by assuming a solution in the form

$A=p \exp (\lambda \eta), \quad B=q \exp (\lambda \eta), \quad A_{d}=p \exp (\lambda \eta-\epsilon \lambda \tau), \quad B_{d}=q \exp (\lambda \eta-\epsilon \lambda \tau)$, 
where $p$ and $q$ are constants. After substituting them into Eqs. (95), one can derive:

$$
\left[\begin{array}{cc}
-\lambda-\beta \sin \frac{\tau}{2} \exp (-\epsilon \lambda \tau) & -\beta \cos \frac{\tau}{2} \exp (-\epsilon \lambda \tau)+\delta_{1}-\frac{\alpha}{2} \\
\beta \cos \frac{\tau}{2} \exp (-\epsilon \lambda \tau)-\delta_{1}-\frac{\alpha}{2} & -\lambda-\beta \sin \frac{\tau}{2} \exp (-\epsilon \lambda \tau)
\end{array}\right]\left[\begin{array}{l}
p \\
q
\end{array}\right]=\left[\begin{array}{l}
0 \\
0
\end{array}\right]
$$

For a nontrivial solution $(p, q)$, we require the determinant to vanish, which yields:

$\lambda^{2}+2 \beta \sin \frac{\tau}{2} \lambda \exp (-\epsilon \tau \lambda)-2 \beta \delta_{1} \cos \frac{\tau}{2} \exp (-\epsilon \tau \lambda)+\beta^{2} \exp (-2 \epsilon \tau \lambda)+\delta_{1}^{2}-\frac{\alpha^{2}}{4}=0$

For the transition between stability and instability, we set $\lambda=0$, giving the following value for $\delta_{1}$ :

$$
\delta_{1}=\beta \cos \frac{\tau}{2} \pm \frac{1}{2} \sqrt{\alpha^{2}-\left(\mu+2 \beta \sin \frac{\tau}{2}\right)^{2}} .
$$

This further gives the following expression for the transition curves of the 2:1 resonance tongue:

$$
\delta=\frac{1}{4}+\epsilon \beta \cos \frac{\tau}{2} \pm \frac{\epsilon}{2} \sqrt{\alpha^{2}-\left(\mu+2 \beta \sin \frac{\tau}{2}\right)^{2}}
$$

The delay term in Eq. (85) produces an effective damping effect [32], where the instability tongue detaches from the $\delta$ axis as in the case of a linearly viscously damped Mathieu equation $(\beta=0)$. Figure 7 a shows a three dimensional plot of the first tongue in the parameter space $(\delta, \tau, \epsilon)$ for the undamped case $\mu=0$. Figure $7 \mathrm{~b}$ also has a form of a three-dimensional stability chart in the parameter space $(\delta, b, \epsilon)$ of the delayed undamped Mathieu equation with $\tau=2 \pi$, which is redrawn based on the one obtained in [33] by utilizing the method of exponential multipliers. The shaded triangles are stable and represent the intersections of the regions outside the instability tongues with the planes $\epsilon=0$ and $\epsilon=2$.

Numerical techniques for stability analysis of DDEs using infinite dimensional Floquet theory have also been developed, where the Floquet transition matrix becomes a compact infinite dimensional monodromy operator. A basis of expansion is chosen and the operator is then approximated in a finite number of dimensions as a square matrix whose eigenvalues or Floquet multipliers determine the stability of the DDE. Such techniques include temporal 
finite element analysis (TFEA) and Chebyshev polynomial expansion [34]. In contrast to perturbation methods, these techniques do not require the parameters to be small. The TFEA method consists of using finite elements in time, arranged so that the position and velocity at the beginning and end of each element is matched to the corresponding values one period earlier. The solution on each element is written as a linear combination of polynomials (trial functions). In the Chebyshev polynomial expansion method, the DDE is written in the form of an integral equation, and the state vector, the periodic coefficients and the initial function are all expressed as shifted Chebyshev polynomials. The method results in a finite-dimensional operator that relates the Chebyshev coefficient vector in a given time interval to that in the previous interval.

\subsection{Delayed nonlinear Mathieu's equation}

Now we show the effect of adding delay to a nonlinear Mathieu equation, in particular the occurrence of Hopf bifurcation in such a system. We consider the following delayed nonlinear Mathieu equation:

$$
\ddot{x}+(\delta+\epsilon \alpha \cos t) x+\epsilon \gamma x^{3}=\epsilon \beta x(t-\tau),
$$

and we investigate the occurrence of Hopf bifurcation using the two variable expansion method. The solution at order $\epsilon^{0}$ takes the form of Eq. (91).

Carrying out the method as in the previous section, we end up with the following slow flow:

$$
\begin{aligned}
& \frac{d A}{d \eta}=\left[\frac{3}{4} \gamma\left(A^{2}+B^{2}\right)-\frac{\alpha}{2}+\delta_{1}\right] B-\beta A_{d} \sin \frac{\tau}{2}-\beta B_{d} \cos \frac{\tau}{2} \\
& \frac{d B}{d \eta}=\left[-\frac{3}{4} \gamma\left(A^{2}+B^{2}\right)-\frac{\alpha}{2}-\delta_{1}\right] A+\beta A_{d} \cos \frac{\tau}{2}-\beta B_{d} \sin \frac{\tau}{2}
\end{aligned}
$$

Hopf bifurcation occurs when the origin loses stability with birth of a limit cycle. Therefore, to approximate the parameters causing Hopf bifurcation we linearize Eqs. (101), (102) about the origin $(0,0)$, which gives the same equations as (93), (94). We then proceed as in the previous section, and we set $\lambda=i \omega$ for a Hopf bifurcation and use Euler's formula $\exp (-i \omega \epsilon \tau)=$ $\cos (\omega \epsilon \tau)-i \sin (\omega \epsilon \tau)$. Separating real and imaginary parts, we obtain 


$$
\begin{aligned}
& \beta^{2}\left(\sin \frac{\tau}{2}\right)^{2} \cos (2 \epsilon \tau \omega)+\beta^{2}\left(\cos \frac{\tau}{2}\right)^{2} \cos (2 \epsilon \tau \omega)+2 \beta \omega \sin \frac{\tau}{2} \sin (\epsilon \tau \omega) \\
& -2 \delta_{1} \beta \cos \frac{\tau}{2} \cos (\epsilon \tau \omega)-\omega^{2}-\frac{\alpha^{2}}{4}+\delta_{1}^{2}=0, \\
& -\beta^{2}\left(\sin \frac{\tau}{2}\right)^{2} \sin (2 \epsilon \tau \omega)-\beta^{2}\left(\cos \frac{\tau}{2}\right)^{2} \sin (2 \epsilon \tau \omega)+2 \delta_{1} \beta \cos \frac{\tau}{2} \sin (\epsilon \tau \omega) \\
& +2 \beta \omega \sin \frac{\tau}{2} \cos (\epsilon \tau \omega)=0 .
\end{aligned}
$$

In this analysis we do not approximate the delayed variable such that:

$$
A_{d}=A(\eta-\epsilon \tau) \approx A(\eta)+O(\epsilon), \quad B_{d}=B(\eta-\epsilon \tau) \approx B(\eta)+O(\epsilon),
$$

as in many authors' work (see, for example, Morrison [32], Atay [35], Wirkus [36]), which results in the slow flow being ODEs rather than DDEs. It is argued that such a step is justified if the product $\epsilon \tau$ is small. Here instead we retain the delayed variables $A_{d}, B_{d}$ in the slow flows Eqs. (93), (94) and Eqs. (101), (102), as in [37]. Next, the two characteristic Eqs. (103), (104) are solved for the pair $(\omega, \tau)$. A perturbation schema is used by setting

$$
\begin{aligned}
\omega_{c r} & =\sum_{n=0}^{N} \epsilon^{n} \omega_{n}=\omega_{0}+\epsilon \omega_{1}+\epsilon^{2} \omega_{2}+\ldots, \\
\tau_{c r} & =\sum_{n=0}^{N} \epsilon^{n} \tau_{n}=\tau_{0}+\epsilon \tau_{1}+\epsilon^{2} \tau_{2}+\ldots
\end{aligned}
$$

After inserting Eqs. (105), (106) into Eqs. (103), (104), and Taylor expanding the trig functions with respect to the small parameter $\epsilon<<1$, the terms of equal order of $\epsilon$ are equated together to obtain the values of $\omega_{n}$ and $\tau_{n}$ which are given in Appendix D. For the order zero in $\epsilon$, the expressions of $\omega_{0}$ and $\tau_{0}$ are as follows:

$$
\begin{aligned}
\omega_{0} & =\frac{\sqrt{\left(2 \beta-\alpha+2 \delta_{1}\right)\left(2 \beta+\alpha+2 \delta_{1}\right)}}{2}, \\
\tau_{0} & =2 \pi,
\end{aligned}
$$

and the value of $\tau_{0}$ corresponds to the critical time delay obtained if the delayed variables $A_{d}$ and $B_{d}$ are replaced by $A$ and $B$ resulting in an ODE 
slow flow, see [32]. Figure 8 shows the Hopf bifurcation curve, Eq. (106), up to order $\epsilon^{3}$ for the fixed parameters $\epsilon=0.05, \beta=3 / 5, \alpha=1$. Adding delay to a nonlinear Mathieu equation results in creating a limit cycle for some delay parameter values.

The delayed Mathieu equation has application to the dynamics of the Synchrotron, a circular particle accelerator [38]. As a group of electrons (called a "bunch") rotates around the accelerator, it leaves an electrical disturbance behind it. The delay term in the governing differential equation comes from the force on the bunch as it passes through its position one cycle earlier.

\section{Fractional Mathieu's equation}

This section is concerned with the Mathieu equation that contains a fractional derivative term $D^{\alpha} x$ and has the form [39]:

$$
\ddot{x}+c D^{\alpha} x+(\delta+\epsilon \cos t) x=0 .
$$

The term $D^{\alpha} x$ is the order $\alpha$ derivative of $x$, where $0<\alpha<1$. As this parameter $\alpha$ varies from 0 to 1 , the fractional derivative term actually combines the effects of stiffness and damping into a single term. Its mathematical definition includes the following integral presentation [39], [40]:

$$
D^{\alpha} x=\frac{1}{\Gamma(1-\alpha)} \frac{d}{d t} \int_{0}^{t}(t-u)^{-\alpha} x(u) d u
$$

where $\Gamma$ is the Euler Gamma function. This expression can be simplified by taking $v=t-u$, giving

$$
D^{\alpha} x=\frac{1}{\Gamma(1-\alpha)} \frac{d}{d t} \int_{0}^{t} v^{-\alpha} x(t-v) d v
$$

Carrying out the differentiation under the integral sign and adopting $x(0)=0$ [40], the final formula that will be used subsequently is derived:

$$
D^{\alpha} x=\frac{1}{\Gamma(1-\alpha)} \int_{0}^{t} v^{-\alpha} x^{\prime}(t-v) d v
$$

To obtain approximate expressions for transition curves in the fractional Mathieu equation (109), harmonic balancing is used. From Floquet theory 
it is known that on the transition curves there exist periodic solutions to Eq. (109) with period $\pi$ or $2 \pi$. Thus, in order to obtain an approximation for the first transition curves, we posit a truncated Fourier series:

$$
x(t)=A \cos \frac{t}{2}+B \sin \frac{t}{2} .
$$

One can present the integral from Eq. (112) as follows:

$$
\begin{aligned}
\int_{0}^{t} v^{-\alpha} x^{\prime}(t-v) d v= & \frac{1}{2} \cos \frac{t}{2} \int_{0}^{t}\left(B \cos \frac{v}{2}+A \sin \frac{v}{2}\right) d v+ \\
& \frac{1}{2} \sin \frac{t}{2} \int_{0}^{t}\left(B \sin \frac{v}{2}-A \cos \frac{v}{2}\right) d v \\
= & \frac{1}{2^{\alpha}} \cos \frac{t}{2}\left(B I_{c}+A I_{s}\right)+\frac{1}{2^{\alpha}} \sin \frac{t}{2}\left(B I s-A I c c_{1} 1,\right.
\end{aligned}
$$

where

$$
I_{c}=\int_{0}^{t / 2} w^{-\alpha} \cos w d w, \quad I_{s}=\int_{0}^{t / 2} w^{-\alpha} \sin w d w .
$$

These can be evaluated in the limit $t \rightarrow \infty$ :

$$
I_{c}=\Gamma(1-\alpha) \sin \frac{\alpha \pi}{2}, \circlearrowleft I_{s}=\Gamma(1-\alpha) \cos \frac{\alpha \pi}{2} .
$$

Thus, restricting attention to the large $t$ limit, the following expression for the fractional derivative (109) is derived:

$$
D^{\alpha} x=\frac{1}{2^{\alpha}} \cos \frac{t}{2}\left(B \sin \frac{\alpha \pi}{2}+A \sin \frac{\alpha \pi}{2}\right)+\frac{1}{2^{\alpha}} \sin \frac{t}{2}\left(B \sin \frac{\alpha \pi}{2}-A \sin \frac{\alpha \pi}{2}\right) .
$$

Substituting Eqs. (113) and (118) into Eq. (109), collecting the terms as in harmonic balancing, equating to zero coefficients of $\sin \frac{t}{2}$ and $\cos \frac{t}{2}$, and eliminating $A$ and $B$ from the resulting two equations, the following expression for the first transition curves are obtained:

$$
\delta=\frac{1}{4}-\frac{c}{2^{\alpha}} \cos \frac{\alpha \pi}{2} \pm \frac{\sqrt{2^{2 \alpha} \epsilon^{2}-4 c^{2} \sin ^{2} \frac{\alpha \pi}{2}}}{2^{\alpha+1}} .
$$


As a check, one substitutes $\alpha=1$ in Eq. (119), in which case the fractional derivative in Eq. (109) becomes an ordinary first derivative and we obtain the damped Mathieu equation (45), while Eq. (119) simplifies to Eq. (52).

The expression for the transition curve (119) indicates that a change in the order $\alpha$ of the fractional derivative affects the shape and location of the transition curves: it moves it along the horizontal axis as the overall stiffness coefficient changes; it also lifts it up as in the case of linear viscous damping (Figure 9).

It is of interest to find the location of the lowest point on the transition curve, which represents the minimum quantity of forcing amplitude necessary to produce instability. Let us refer to this minimum value of $\epsilon$, for a given value of $\alpha$, as $\epsilon_{\min }$. Differentiating Eq. (119), one can find this value to be:

$$
\epsilon_{\min }=c \frac{\sin \frac{\alpha \pi}{2}}{2^{\alpha-1}},
$$

which is plotted in Figure 10. The greatest effect is observed where this curve achieves its maximum, which is obtained for

$$
\alpha^{*}=\frac{2}{\pi} \arctan \frac{\pi}{2 \ln 2} \approx 0.735
$$

yielding $\epsilon^{*} \approx 1.099 c$. Figure 10 also implies that when $\alpha$ lies in the range $(0.5$, 1 ), the values for $\epsilon_{\min }$ are all greater than the corresponding minimum for the linearly viscously damped Mathieu equation. Thus, the damping effect of the fractional derivative term for $0.5<\alpha<1$, is greater than that of the linearly viscously damped Mathieu equation.

In [41], one more fractional term is added to the Mathieu equation (109) a fractional delay derivative term $D^{\Lambda} x(t-\tau)$, so that the governing equation has the form

$$
\ddot{x}(t)+c D^{\alpha} x(t)+(\delta+\epsilon \cos t) x(t)=b D^{\Lambda} x(t-\tau),
$$

where $\tau>0$ is the time delay, $0<\alpha<1$ and $0<\Lambda<1$ are fractional orders of $x(t)$ and its delayed value, while $b$ and $c$ are real constants. Using the algorithm presented above, one can derive the following expression for the first transition curves [41]:

$\delta=\frac{1}{4}-\frac{c}{2^{\alpha}} \cos \frac{\alpha \pi}{2}+\frac{b}{2^{\Lambda}} \cos \frac{\Lambda \pi-\tau}{2} \pm \sqrt{\frac{1}{4} \epsilon^{2}-\left(\frac{c}{2^{\alpha}} \sin \frac{\alpha \pi}{2}-\frac{b}{2^{\Lambda}} \sin \frac{\Lambda \pi-\tau}{2}\right)^{2}}$. 
This equation reduces to (119) for $b=0$ and to (99) for $\alpha=1$ and $\Lambda=0$.

Given the number of the system parameters, one can use them to affect appropriately the shape and location of the transition curves. A minimum of forcing amplitude necessary to produce instability is now given by $\epsilon_{\min }=$ $\left|2^{1-\alpha} c \sin \frac{\alpha \pi}{2}-2^{1-\Lambda} b \sin \frac{\Lambda \pi-\tau}{2}\right|$. The greatest effect of the fractional order $\Lambda$ on it (when other parameters are fixed) occurs for the value that might be expressed as

$$
\Lambda^{*} \approx 0.735+\frac{\tau}{\pi}
$$

where $\tau$ should be such that $\Lambda^{*}<1$.

Figure 11 illustrates these findings in terms of the first transition curves plotted for the cases related to Eqs. (123) and (124). Two cases shown for different system parameters illustrate that the delay can put down (Figure 11a) but also can lift up the transition curves (Figure 11b).

\section{Quasiperiodic Mathieu's equation}

In this section we consider the quasiperiodic Mathieu equation:

$$
\ddot{x}+(\delta+\epsilon \cos t+\epsilon \cos \omega t) x=0 .
$$

For a given set of parameters $(\delta, \omega, \epsilon)$, Eq. (125) is said to be stable (S) if all solutions are bounded, and unstable (U) otherwise.

As a first approximation we may think of the instabilities occurring in Eq. (125) as consisting of the union of the instabilities of each of the two equations:

$$
\ddot{x}+(\delta+\epsilon \cos t) x=0
$$

and

$$
\ddot{x}+(\delta+\epsilon \cos \omega t) x=0 .
$$

See Figure 12 where $\omega$ is fixed and $\epsilon$ and $\delta$ are varied. We may also plot the same results by fixing $\epsilon$ and varying $\omega$ and $\delta$, as shown in Figure 13 .

The approximate nature of this scheme for obtaining the stability chart of Eq. (125) is revealed by comparing with the results of direct numerical integration. In [42], [44], Eq. (125) was numerically integrated forward in time from arbitrarily chosen initial conditions at $t=0$ up to $t=20,000$. 
At each step the amplitude $\sqrt{x(t)^{2}+\dot{x}(t)^{2}}$ was computed and a motion was judged to be unstable if its amplitude became greater than a million times its initial value for any $t$ between 0 and 20,000, and stable otherwise. This results in Figure 14a.

Figure 14b shows an enlargement of Figure 14a around $\delta=0.25, \omega=1$, and Figure 14c shows an enlargement of Figure 14a around $\delta=0.25, \omega=0.1$.

Figures 14a-c refer to Eq. (125) with $\epsilon=0.1$. To see the effect of changing $\epsilon$, Figures $15 \mathrm{a}$-e show regions of the stability chart for varying values of $\epsilon$. Note that Figures 15a-c, which correspond to $\epsilon=0.01,0.05$ and 0.1 , lie in the region of Figure 15d which is bounded by thick straight lines. The reason for this is that as $\epsilon$ increases, the black (stable) regions disappear, so we show a larger region of the parameter space for $\epsilon=0.5$ and $\epsilon=1$, otherwise there would be very little to show (it would be almost all white). These figures show the complexity and fractal nature of the stability regions for the quasiperiodic Mathieu equation (125). There have been numerous papers which have used perturbation methods to examine the structure of the stability charts in the neighborhood of various resonances. For example, the nature of the stability chart near $\delta=0.25, \omega=1$, see Figure 14b, involves a 2:2:1 resonance and has been studied in [45], [46]. The diagram near $\delta=0.25$, $\omega=0.5$, see Figure 14a, involves a 2:1:1 resonance and has been studied in [43]. The region near $\delta=0.25, \omega=0$, see Figure 14a, was studied in [42], [44].

In contrast to these results obtained by perturbation methods, a recent paper by Sharma and Sinha [47] has used an approximate numerical approach based on Floquet theory. Although there is no Floquet theory for quasiperiodic systems, these authors have replaced the quasi-periodic system with a periodic system which has a large principal period, and then used Floquet theory on the resulting periodic system.

In all of these studies of Eq. (125), instability means unboundedness as time $t$ goes to infinity. This is because Eq. (125) is linear. The effect of adding nonlinear terms to Eq. (125) was considered in [48], where the following nonlinear quasiperiodic Mathieu equation was studied:

$$
\ddot{x}+(\delta+\epsilon \cos t+\epsilon \cos \omega t) x+\alpha x^{3}=0 .
$$

In [48] a perturbation scheme using Jacobi elliptic functions was combined 
with KAM theory to determine the global behavior of Eq. (128)

\section{Mathieu's equation with elliptic-type exci- tation}

Let us consider a pendulum from Case 1 discussed in Section 2. Instead of prescribing the motion of its support, it is assumed that its acceleration is $A \operatorname{cn}(\Omega t \mid m)$, where $A, \Omega, m$ are constants. The corresponding equation for small oscillations has the form

$$
\ddot{x}+(\delta+\epsilon \operatorname{cn}(t \mid m)) x=0,
$$

where $\delta=g /\left(L \Omega^{2}\right)$ and $\epsilon=A /\left(L \Omega^{2}\right)$. So, it is seen now that the parametric excitation existing in Eq. (129) has the form of the Jacobi elliptic function, which is a periodic two-argument function: the coefficient in front of $t$ in the first argument is equal to unity, which means that the frequency of the elliptic function is unity; the constant $m$ appearing in the second argument represents the so-called elliptic parameter [49]-[52].

This type of excitation can be considered as a generalisation of the Cosine excitation in the classical Mathieu equation (1). Namely, when $m=0$ the Jacobi cn function transforms into the Cosine function: $\operatorname{cn}(t \mid m)=\cos t$ [50]. Previous investigations have been concerned with other types of elliptic-type functions. For example, Lamé's equation includes a square of the Jacobi sn function as a periodically varying coefficient in front of a linear term. Its origin is in the theory of the potential of an ellipsoid [2], [4]. This equation is characterized by the fact that under some condition related to the amplitude of the parametric excitation, coexistence [1] can take place, which means that an infinite number of possible tongues of instability does not occur. It is interesting that Lamé's equation can be transformed into Incé's equation which has time-varying inertial, damping and stiffness coefficients that are expressed in terms of trigonometric functions. Generalizations of Lame's equation comprise [2]: the Hermite elliptic equation, whose damping term contains all three basic Jacobi functions (sn, cn and dn) and the Picard elliptic equation, which has a similar damping term as the Hermite elliptic equation, but its stiffness term is constant.

Let us go back to the elliptic excitation and its form in Eq. (129) and define the values of the elliptic parameter $m$ that will be considered. Three 
cases can be distinguished [53] (indicated subsequently by the corresponding index): Case I corresponds to $m_{\mathrm{I}}<0$; Case II includes the domain $0<m_{\mathrm{II}}<$ 1 (this case was also analysed in [54]); Case III encompasses $m_{\mathrm{III}}>1$. Note that the value $m=1$ is not of interest here as the cn elliptic function turns then into the hyperbolic secant (the inverse of hyperbolic cosine), which is not periodic.

To provide some interpretation of the elliptic-type excitation given for Cases I-III, its Fourier series expansion is used [49]:

$$
\operatorname{cn}(t \mid m)=\frac{2 \pi}{K \sqrt{m}} \sum_{N=1}^{\bar{N}} \frac{q^{N-1 / 2}}{1+q^{2 N-1}} \cos \left((2 N-1) \frac{\pi}{2 K} t\right),
$$

with $K$ being the complete elliptic integral of the first kind, while $K^{\prime}$ is its associated complete elliptic integral of the first kind. Note that, by the definition [49], $K$ depends on the elliptic parameter as follows

$$
K \equiv K(m)=\int_{0}^{\pi / 2} \frac{d \theta}{\sqrt{1-m \sin ^{2} \theta}},
$$

while $K^{\prime}=K(1-m)$.

The function $q$ in Eq. (130) is the so-called Nome, defined as a function of $K$ and $K^{\prime}$ :

$$
q=\exp \left(-\frac{\pi K^{\prime}}{K}\right)
$$

Equation (130) yields the conclusion that the elliptic-type excitation can be interpreted as a multi-cosine excitation whose harmonics have their amplitudes and frequencies coupled through the parameter $m$.

The expansion (130) holds both for $m=m_{\mathrm{I}}$ and $m=m_{\mathrm{II}}$, and it can be represented also as follows:

$$
\begin{aligned}
\operatorname{cn}\left(t \mid m_{\mathrm{I}, \mathrm{II}}\right) & =\sum_{N=1}^{\bar{N}} C_{N} \cos \left((2 N-1) \frac{\pi}{2 K_{\mathrm{I}, \mathrm{II}}} t\right) \\
C_{N} & =\frac{2 \pi}{K_{\mathrm{I}, \mathrm{II}} \sqrt{m_{\mathrm{I}, \mathrm{II}}}} \frac{q_{\mathrm{I}, \mathrm{II}}^{N-1 / 2}}{1+q_{\mathrm{I}, \mathrm{II}}^{2 N-1}},
\end{aligned}
$$

where $K_{\mathrm{I}}=K\left(m_{\mathrm{I}}\right), K_{\mathrm{II}}=K\left(m_{\mathrm{II}}\right), q_{\mathrm{I}}=q\left(K_{\mathrm{I}}^{\prime}, K_{\mathrm{I}}\right)$ and $q_{\mathrm{II}}=q\left(K_{\mathrm{II}}^{\prime}, K_{\mathrm{II}}\right)$ 
To deal with a real square root which occurs for negative values of the elliptic parameter, i.e. for $m=m_{\mathrm{I}}$, Eq. (133) can also be presented as

$$
\begin{aligned}
\operatorname{cn}\left(t \mid m_{\mathrm{I}}\right) & =\sum_{N=1}^{\bar{N}} C_{\mathrm{I} N} \cos \left((2 N-1) \frac{\pi}{2 K_{\mathrm{I}}} t\right), \\
C_{\mathrm{I} N} & =\frac{2 \pi}{K_{\mathrm{I}} \sqrt{-m_{\mathrm{I}}}} \frac{(-1)^{N-1}\left(-\hat{q}_{\mathrm{I}}\right)^{N-1 / 2}}{1+\hat{q}_{\mathrm{I}}^{2 N-1}}, \\
\hat{q}_{\mathrm{I}} & =-\exp \left(-\frac{\pi K^{\prime}\left(\frac{1}{1-m_{\mathrm{I}}}\right)}{\sqrt{1-m_{\mathrm{I}}} K\left(m_{\mathrm{I}}\right)}\right)
\end{aligned}
$$

Equations (133) and (135) show clearly that both series expansions encompass odd harmonics, with the frequency and their amplitudes depending on $m$. In Case I, the coefficients of harmonics have an alternating sign, while in Case II they are all positive. The corresponding period can be calculated from Eq. (133) as:

$$
T_{\mathrm{I}, \mathrm{II}}=\frac{2 \pi}{\frac{\pi}{2 K_{\mathrm{I}, \mathrm{II}}}}=4 K_{\mathrm{I}, \mathrm{II}} \equiv 4 K\left(m_{\mathrm{I}, \mathrm{II}}\right),
$$

which is the well-known period of the Jacobi cn function for such parameter values [49].

Case III, corresponding to $m_{\mathrm{III}}>1$, can be examined by transforming the cn function into the dn elliptic function as follows [49]

$$
\operatorname{cn}\left(t \mid m_{\mathrm{III}}\right)=\operatorname{dn}\left(\sqrt{m_{\mathrm{III}}} t \mid \frac{1}{m_{\mathrm{III}}}\right) .
$$

This can be further expressed as the following Fourier series

$$
\begin{aligned}
\operatorname{dn}\left(\sqrt{m_{\mathrm{III}}} t \mid \frac{1}{m_{\mathrm{III}}}\right) & =D_{0}+\sum_{N=1}^{\bar{N}} D_{N} \cos \left(N \frac{\pi \sqrt{m_{\mathrm{III}}}}{K_{\mathrm{III}}} t\right), \\
D_{0} & =\frac{\pi}{2 K_{\mathrm{III}}}, \quad D_{N}=\frac{2 \pi}{K_{\mathrm{III}}} \frac{q_{\mathrm{III}}^{N}}{1+q_{\mathrm{III}}^{2 N}},
\end{aligned}
$$

where 


$$
K_{\mathrm{III}} \equiv K\left(\frac{1}{m_{\mathrm{III}}}\right)
$$

and $q_{\mathrm{III}}=q\left(K_{\mathrm{III}}^{\prime}, K_{\mathrm{III}}\right)$.

The series representation given by Eq. (140) implies that this type of excitation includes both odd and even harmonics. It oscillates around the offset $\pi /\left(2 K_{\mathrm{III}}\right)$, which increases as $m_{\mathrm{III}}$ increases and tends to unity when $m_{\mathrm{III}} \rightarrow \infty$.

The period corresponding to Case III is

$$
T_{\mathrm{III}} \equiv \frac{2 \pi}{\frac{\pi \sqrt{m_{\mathrm{III}}}}{K_{\mathrm{III}}}}=\frac{2 K_{\mathrm{III}}}{\sqrt{m_{\mathrm{III}}}}=\frac{2 K\left(\frac{1}{m_{\mathrm{III}}}\right)}{\sqrt{m_{\mathrm{III}}}} .
$$

Using Eqs. (138) and (143), the period of the cn elliptic excitation is plotted in Figure $16 \mathrm{~N}$ as a function of the elliptic parameter $m$. When $m$ tends to minus infinity or infinity, the period tends to zero. When $m=0$, the period has the well-known value $2 \pi$, as $\operatorname{cn}(t \mid 0)=\cos t$ [49], [51], [52].

The next objective is to investigate the stability of the trivial equilibrium solution $x=0$, i.e. to determine stability charts in the $\delta-\epsilon$ plane of the equation of motion (129) and the effects of the elliptic parameter on the stability chart. The associated transition curves can be obtained from the fact that the unperturbed linear oscillator has a solution of period $2 \pi / \sqrt{\delta}$, which should correspond to the solutions of the excited oscillator where the period of excitation is $T$, i.e. $2 \pi / \sqrt{\delta_{0}}=2 T / n, n=1,2,3, \ldots$ The excitation in the classical Mathieu equation is harmonic with the period $T=2 \pi$, and the zero points are given by $\delta_{0}=n^{2} / 4$ (see Section 3.1.3). Following the same approach in Case I and II, the period of the excitation is given by Eq. (138), so that the zero-points are

$$
\delta_{0 \mathrm{I}, \mathrm{II}}=\frac{\pi^{2} n^{2}}{16 K_{\mathrm{I}, \mathrm{II}}^{2}} .
$$

In Case III, the period is given by Eq. (143), and the zero-points are defined by

$$
\delta_{0 \mathrm{III}}=\frac{\pi^{2} n^{2} m_{\mathrm{III}}}{4 K_{\mathrm{III}}^{2}}
$$


Numerically obtained stability charts found by applying the Floquet theory (see Section 3.1.2) corresponding to $m_{\mathrm{I}}=-0.5, m_{\mathrm{II}}=0.5$ and $m_{\mathrm{III}}=1.5$ are presented in Figure 17. These numerical results are labelled as shaded areas. Comparing Figures 17a-c to the stability chart of the Mathieu equation shown in Figure 3, it is seen that both the location, shape and size of the instability tongues change with $m$ : for $m<0$, the instability tongues are relocated to the right; for $0<m<1$, the instability tongues are relocated to the left, i.e. to smaller values of $\delta$; for $m>1$, they are more profoundly relocated to higher values of $\delta$ as well as inclined to the left.

The next task is to determine the analytical expression for the transition curves. Harmonic balancing is to be utilized for this purpose. First, the cn elliptic function is replaced by the corresponding Fourier series in the equation of motion. So, in Case I and II, the series given by Eq. (133) is used in Eq. (129), yielding

$$
\ddot{x}+\left(\delta+\epsilon \sum_{N=1}^{\bar{N}} C_{N} \cos \left((2 N-1) \frac{\pi}{2 K_{\mathrm{I}, \mathrm{II}}} t\right)\right) x=0 .
$$

The solution for motion is assumed in the form that includes a set of harmonics

$$
x(t)=\sum_{n=0}^{\bar{N}} a_{n} \cos \left(n \frac{\pi}{T} t\right)+b_{n} \sin \left(n \frac{\pi}{T} t\right) .
$$

Substituting the solution (147) with the period $T_{\mathrm{I}, \mathrm{II}}$ (138) into Eq. (146) and applying harmonic balancing as explained in Section 3.1.3, four sets of algebraic equations with respect to the coefficients $a_{\mathrm{I}, \mathrm{II} e v e n}, a_{\mathrm{I}, \mathrm{IIodd}}, b_{\mathrm{I}, \text { IIeven }}$ and $b_{\mathrm{I}, \text { IIodd }}$ are derived. For a nontrivial solution, the corresponding determinants must vanish, leading to the so-called Hill's determinants, which are given in Appendix E.

In Case III, the series (140) is used in Eq. (129)

$$
\ddot{x}+\left(\delta+\epsilon D_{0}+\epsilon \sum_{N=1}^{\bar{N}} D_{n} \cos \left(N \frac{\pi \sqrt{m_{\mathrm{III}}}}{K_{\mathrm{III}}} t\right)\right) x=0 .
$$

Applying the same procedure related to harmonic balancing of Eq. (148) with the solution (147) and the period (143), Hill's determinants for $a_{\mathrm{IIIeven}}$, $a_{\text {IIIodd }}, b_{\text {IIIeven }}$ and $b_{\text {IIIodd }}$ are derived. They are also included in Appendix 
E. This Case II was also studied in [54] by introducing a new time in the form of the Jacobi amplitude, transforming the original equation into the equation with time-varying coefficients, all of which are expressed in terms of trigonometric functions. Harmonic balancing is then applied and Hill's determinant derived.

By using the determinants derived in this approach, transition curves are plotted in Figure 17Na-c for different values of the parameter $m$ for all three Cases considered. These results are labelled by red solid lines, and represent the boundaries of the shaded numerically obtained instability regions, which confirms their accuracy.

The Hill's determinant $a_{\mathrm{I}, \mathrm{II} \text { even }}-b_{\mathrm{I}, \mathrm{II} \text { odd }}$ given in Appendix $\mathrm{E}$ can be used to derive analytical expressions for transition curves in the form $\delta=\delta_{0}(m)+$ $\epsilon \delta_{1}(m)+\epsilon^{2} \delta_{2}(m)$ [53]. Considering the case $\tilde{N}=10$ and truncating all the determinants to the dimension $3 \times 3$, the following expressions for the zero-transition curve and two subsequent pairs are obtained [53]:

$$
\begin{gathered}
\delta=-\frac{2 K_{\mathrm{I}, \mathrm{II}}^{2} C_{1}^{2}}{\pi^{2}} \epsilon^{2}, \\
\delta=\frac{\pi^{2}}{16 K_{\mathrm{I}, \mathrm{II}}^{2}}-\frac{C_{1}}{2} \varepsilon-\frac{K_{\mathrm{I}, \mathrm{II}}^{2}\left(3 C_{1}^{2}+C_{2}^{2}\right)}{6 \pi^{2}} \epsilon^{2}, \\
\delta=\frac{\pi^{2}}{16 K_{\mathrm{I}, \mathrm{II}}^{2}}+\frac{C_{1}}{2} \epsilon-\frac{K_{\mathrm{I}, \mathrm{II}}^{2}\left(3 C_{1}^{2}+C_{2}^{2}\right)}{6 \pi^{2}} \epsilon^{2}, \\
\delta=\frac{\pi^{2}}{4 K_{\mathrm{I}, \mathrm{II}}^{2}}-\frac{K_{\mathrm{I}, \mathrm{II}}^{2}\left(C_{1}-C_{2}\right)^{2}}{3 \pi^{2}} \epsilon^{2}, \\
\delta=\frac{\pi^{2}}{4 K_{\mathrm{I}, \mathrm{II}}^{2}}+\frac{K_{\mathrm{I}, \mathrm{II}}^{2}\left(5 C_{1}^{2}-2 C_{1} C_{2}-C_{2}^{2}\right)}{3 \pi^{2}} \epsilon^{2} .
\end{gathered}
$$

Setting $\epsilon=0$, one can easily recognized that they all emanate from the exact locations given by Eqs. (144) and (145). These results are further used to get a more detailed overview on how the stability charts change with the elliptic parameter $-5 \leq m \leq 5$. Using these results, an animation (Animation 1) is created, which is given as Supplementary Material to this article. It illustrates clearly that for $m<1$, the instability regions move towards lower values of $\delta$ and become more dense when $m$ approaches unity. This conclusion is also given in [54] for $0<m<1$. In the case $m>1$, the instability tongues are oblique and shift towards higher values of $\delta$, becoming less dense as $m$ increases. 


\section{Discussions and conclusions}

This tutorial review article has been concerned with one of the archetypical equations of Nonlinear Vibrations Theory - Mathieu's equation and its stability chart. This classical equation corresponds to a linear second-order ordinary differential equation, whose stiffness coefficient contains a constant term $\delta$ and a time-varying (periodic) forcing $\epsilon \cos t$. Depending on the combination of this constant term $\delta$ and the forcing amplitude $\epsilon$, the motion can stay bounded (this case is referred to as stable) or unbounded (this case is referred to as unstable). The corresponding graphical presentation in the $\delta-\epsilon$ plane has the form of the so-called stability chart with regions of stability and regions of instability (tongues) separated by pairs of transition curves, along which the response has a periodic character. It has been shown how these transition curves and the points from which they emanate can be obtained in several different ways: by using a perturbation method, Floquet theory and harmonic balancing. It has also been shown how periodic responses along the transition curves can be expressed in terms of Mathieu's functions.

This article has also presented some historical facts about early investigations of Mathieu's equation, rederiving and redrawing some of the key findings, including the very first stability chart as done by Ince [15].

The subsequent analyses have been concerned with various extensions or generalizations of Mathieu's equation in terms of geometric, damping terms, delays, fractional and excitation terms as well as the differences between the resulting stability charts and their characteristics with respect to the one for classical Mathieu's equation. First, it has been demonstrated that linear viscous damping lifts up the tongue and that there is a certain minimal forcing amplitude needed for instability to occur. Then, two cases of nonlinearity in Mathieu's equation have been dealt with: geometric nonlinearity and damping nonlinearity. In general, the existence of the nonlinear term causes the resonance to detune as the amplitude grows, resulting in a periodic motion with finite amplitude inside the tongues, at least for small $\epsilon$. The former nonlinear case examined is related to the hardening cubic type nonlinearity and it has been shown that two pitchfork bifurcations appear associated with the first tongue: when the parameter $\delta$ is quasistatically decreased across the right transition curve while $\epsilon$ is kept fixed, the trivial solution becomes unstable and simultaneously a stable 2:1 subharmonic motion is born; when the left transition curve is crossed, the trivial solution becomes stable again, and an unstable 2:1 subharmonic is born. In case of softening nonlinearity, 
a similar sequence of bifurcations takes place, but the subharmonic motions are born as $\delta$ increases quasistatically through the first tongue. The second case with nonlinearity has been concerned with quadratic damping. The first order averaging carried out has given that there is an attractive period- 2 subharmonic motion inside the first tongue for small $\epsilon$. However, the amplitude of the limit cycles in this second tongue has been found to depend on the value of the parameter $\epsilon$. Numerical investigations have revealed the existence of different Poincaré map portraits in the part of the stability chart investigated: outside the first instability regions, the origin is always stable; inside the instability regions, the origin is unstable; inside the $2: 1$ region the two spiral singularities exist in the Poincaré map representing a single period $4 \pi$ motion, whereas in the $1: 1$ region they represent two period $2 \pi$ motions; in certain parts, supercritical pitchfork bifurcations have been detected to occur, among which is the case of two coexisting outermost periodic orbits the stable and unstable period $4 \pi$ orbits - coalescing and being destroyed in a saddle-node bifurcation, which does not take place at the origin.

It has been found that the delay term in Mathieu's equation produces an effective damping effect where the first instability tongue detaches from the $\delta$ axis as in the case of a linearly viscously damped Mathieu equation. In case Mathieu's equation contains cubic geometric nonlinearity, adding delay results in creating a limit cycle for some delay parameter values.

Fractional Mathieu's equation has also been considered. As the fractional derivative term actually combines the effects of stiffness and damping into a single term, it has been shown that as a result the first tongue shifts along the horizontal axis in the stability chart and it also lifts up. The minimum quantity of forcing amplitude necessary to produce instability has been obtained. It has been found that the damping effect of the fractional derivative term between 0.5 and unity is greater than that of the linearly viscously damped Mathieu equation. In addition, one more fractional term has been added to the Mathieu equation - a fractional delay derivative term. The expression for the first transition curves has been derived as well as the fractional order having the greatest effect on a minimum of forcing amplitude necessary to produce instability. This value of the fractional order depends on the time delay.

Another case of interest has been Mathieu's equation with quasiperiodic forcing, which differs from the classical Mathieu's equation in the existence of the additional parametric forcing of frequency $\omega$. As a first approximation, the instabilities occurring have been seen as consisting of the union of 
the instabilities in two separate equations: classical Mathieu's equation and the one having forcing of frequency $\omega$. Numerical simulations carried out for changeable $\epsilon$ have shown the complexity of the corresponding stability regions, and also their fractal nature.

Finally, the time-varying (periodic) forcing $\epsilon \cos t$ has been replaced by elliptic-type forcing $\operatorname{cn}(t \mid m)$, where $m$ is the elliptic parameter. For $m=0$, this type of forcing simplifies to the cosine one, but in other cases, it can be considered as a periodic multi-cosine excitation whose period and the content change with the elliptic parameter. It has been demonstrated how the content of such multi-cosine excitation changes with the elliptic parameter. These results have been further used to get a detailed overview on how the stability charts change with the elliptic parameter. It has been illustrated that for $m<1$, the instability regions shift towards lower values of $\delta$ and become more dense when $m$ approaches unity. Depending whether $m<0$, or $0<m<1$ the points on the horizontal axis from which the tongues emanate are located on different sides with respect to those in classical Mathieu's equation. In the case $m>1$, the instability tongues are oblique and move towards higher values of $\delta$, becoming less dense as $m$ increases.

Besides giving the overview of the structure and characteristics of stability charts for all these cases, this tutorial review article has also presented a variety of analytical, semi-analytical and numerical approaches to treat them. The reader might want to try to apply/extend them to some other cases which are the combinations of the cases presented herein.

Last but not least, this very first collection of the stability charts of different forms of Mathieu's equation can also have a practical purpose, enabling practitioners to design their system starting from a desirable stability chart and then choosing the system components needed to accomplish it.

NOTE: Although the authors did not explicitly examine symmetry properties of the different forms of the Mathieu equations and the reduced versions of them obtained in the analyses, it is mentioned that the influence of these properties on the bifurcations of solutions considered in this study may be worth looking into, in future work.

Acknowledgement: The involvement of IK was financially supported by the Ministry of Science, Republic of Serbia (Project No. ON174028). This work was partially supported by NSF Grant CMMI-1634664 and by NSF Grant PHY-1549132. 


\section{Appendix A: Extract from Mathieu's study on vibra- tions of an elliptic membrane}

The system under consideration in Mathieu's work from 1868 [8] was an elliptic membrane. The ellipse is centered in the $x-y$ plane and its foci are located at $x= \pm \rho$. Its oscillations in the vertical $w$ directions depend on the position and time, i.e. $w=w(x, y, t)$ and are governed by the wave equation

$$
m^{2}\left(\frac{\partial^{2} w}{\partial x^{2}}+\frac{\partial^{2} w}{\partial y^{2}}\right)=\frac{\partial^{2} w}{\partial t^{2}}
$$

where the constant $m$ depends on the tension force per unit length at the boundary and the mass per unit area.

Separating the variables

$$
w(x, y, t)=u(x, y) v(t),
$$

where $v(t)=\sin (2 \lambda m t)$, the wave equation yields

$$
\frac{1}{u}\left(\frac{\partial^{2} u}{\partial x^{2}}+\frac{\partial^{2} u}{\partial y^{2}}\right)=\frac{1}{u} \nabla^{2} u=\frac{1}{m^{2} v} \frac{d^{2} v}{d t^{2}}=-4 \lambda^{2} .
$$

Now, one can recognize the two-dimensional Helmholtz equation for the displacement $u$ with $\nabla^{2}$ standing for the Laplacian in two dimensions.

As the membrane is elliptic, it is convenient to further use elliptic coordinates. They are introduced in the form

$$
x=\rho \cos \alpha \cosh \beta, \quad y=\rho \sin \alpha \sinh \beta .
$$

Converting the Laplacian into the elliptic coordinates, one can derive

$$
\frac{\partial^{2} u}{\partial x^{2}}+\frac{\partial^{2} u}{\partial y^{2}}+4 \lambda^{2} u=\frac{1}{\rho^{2}\left(\cosh ^{2} \beta-\cos ^{2} \alpha\right)}\left(\frac{\partial^{2} u}{\partial \alpha^{2}}+\frac{\partial^{2} u}{\partial \beta^{2}}\right)+4 \lambda^{2} u=0
$$

and then write this equation down as

$$
\frac{\partial^{2} u}{\partial \alpha^{2}}+\frac{\partial^{2} u}{\partial \beta^{2}}+4 \lambda^{2} \rho^{2}\left(\cosh ^{2} \beta-\cos ^{2} \alpha\right) u=0 .
$$

Separating the variables by assuming $u(\alpha, \beta)=P(\alpha) Q(\beta)$, one can now derive the following equation 


$$
\frac{1}{P} \frac{d^{2} P}{d \alpha^{2}}-2 \lambda^{2} \rho^{2} \cos 2 \alpha=\frac{1}{Q} \frac{d^{2} Q}{d \beta^{2}}+2 \lambda^{2} \rho^{2} \cosh 2 \beta=-R,
$$

where $R$ is a constant.

The equation in $P$ can be expressed as:

$$
\frac{d^{2} P}{d \alpha^{2}}+\left(R-2 h^{2} \cos 2 \alpha\right) P=0
$$

where $h^{2}=\lambda^{2} \rho^{2}$.

The equation of this form is named after Mathieu as 'Mathieu's equation'. Introducing $t=2 \alpha$ and using $P \equiv x, \delta=R / 4, \epsilon=-h^{2} / 2$, it gets an alternative form, which is the starting equation of this article, Eq. (1).

\section{Appendix B: Mathieu functions}

This Appendix contains the method that Mathieu developed to find solutions of Eq. (A.8), composing them as expansions about $h^{2}=0$. So, by setting $h^{2}=0$ into Eq. (A.8), it is easy to identify the following solutions:

$$
\begin{array}{ll}
c_{n}(\alpha)=\cos n \alpha, & R=n^{2}, \quad n=0,1,2, \ldots, \\
s_{n}(\alpha)=\sin n \alpha, & R=n^{2}, \quad n=1,2, \ldots
\end{array}
$$

To treat the case when $h^{2} \neq 0$, we first set $n=1$, assuming:

$$
\begin{aligned}
P\left(h^{2}, \alpha\right) & =\cos \alpha+c_{1}(\alpha) h^{2}+c_{2}(\alpha) h^{4}+c_{3}(\alpha) h^{6}+\ldots, \\
R\left(h^{2}\right) & =1+d_{1} h^{2}+d_{2} h^{4}+d_{3} h^{6}+\ldots,
\end{aligned}
$$

where $c_{i}(\alpha)$ and $d_{i}$ are to be determined under the condition that $P$ is an even and periodic function of $\alpha$ with period $2 \pi$, like $\cos \alpha$. So, substituting these expressions into Eq. (A.8) and grouping the terms next to the same power of $h$, one can derive

$$
\begin{aligned}
& c_{1}^{\prime \prime}(\alpha)+c_{1}(\alpha)=\cos 3 \alpha-\left(d_{1}-1\right) \cos \alpha \\
& c_{2}^{\prime \prime}(\alpha)+c_{2}(\alpha)=2 c_{1}(\alpha) \cos 2 \alpha-d_{2} \cos \alpha-d_{1} c_{1}(\alpha),
\end{aligned}
$$


where the primes denote derivatives with respect to $\alpha$. A periodic solution is sought. However, the last term on the right-hand side of Eq. (B.5) will yield a particular integral growing unboundedly in time and should be removed. This can be achieved by choosing $d_{1}=1$. Solving further Eq. (B.5) gives

$$
c_{1}(\alpha)=-\frac{1}{8} \cos 3 \alpha .
$$

Proceeding in the same way with Eq. (B.6), one finds $d_{2}=-1 / 8$ and

$$
c_{2}(\alpha)=-\frac{1}{64} \cos 3 \alpha+\frac{1}{192} \cos 5 \alpha \text {. }
$$

This process can be further continued to determine other terms in these power series expansions. The solution denoted by $c e_{1}$ is obtained in this way, while the corresponding $R\left(h^{2}\right)$, Eq. (B.4), is labelled by $a_{1}$. These solutions are

$$
\begin{gathered}
c e_{1}\left(h^{2}, \alpha\right)=\cos \alpha-\frac{1}{8} h^{2} \cos 3 \alpha+\frac{1}{64} h^{4}\left(-\cos 3 \alpha+\frac{1}{3} \cos 5 \alpha\right)- \\
\frac{1}{512} h^{6}\left(\frac{1}{3} \cos 3 \alpha-\frac{4}{9} \cos 5 \alpha+\frac{1}{18} \cos 7 \alpha\right)+\ldots, \\
a_{1}\left(h^{2}\right)=1+h^{2}-\frac{1}{8} h^{4}-\frac{1}{64} h^{6}-\frac{1}{1536} h^{8}-\frac{11}{36864} h^{10}+\ldots
\end{gathered}
$$

Using the same algorithm, one can construct $a_{n}\left(h^{2}\right)$ and the respective solutions $c e_{n}\left(h^{2}, \alpha\right)$, which are called cosine-elliptic functions and correspond to $n=0,2,3, \ldots$. The solutions with even $n$ have period $\pi$ and those of odd $n$ have period $2 \pi$. Note that Mathieu functions are included into contemporary symbolic software packages (such as Wolfram Mathematica) and can be used without need to type their definitions/expansions.

Mathieu functions that correspond to $\sin n \alpha$, Eq. (B.2), are called sineelliptic functions and are labelled by $s e_{n}\left(h^{2}, \alpha\right)$. The expansions for $s e_{1}$ and the respective $R\left(h^{2}\right) \equiv b_{1}$ have the form 


$$
\begin{gathered}
s e_{1}\left(h^{2}, \alpha\right)=\sin \alpha-\frac{1}{8} h^{2} \sin 3 \alpha+\frac{1}{64} h^{4}\left(\sin 3 \alpha+\frac{1}{3} \sin 5 \alpha\right)- \\
\frac{1}{512} h^{6}\left(\frac{1}{3} \sin 3 \alpha+\frac{4}{9} \sin 5 \alpha+\frac{1}{18} \sin 7 \alpha\right)+\ldots \\
b_{1}\left(h^{2}\right)=1-h^{2}-\frac{1}{8} h^{4}+\frac{1}{64} h^{6}-\frac{1}{1536} h^{8}-\frac{11}{36864} h^{10}+\ldots
\end{gathered}
$$

The sine-elliptic solutions have period $\pi$ if $n$ is even and period $2 \pi$ if $n$ is odd.

One can use the relationships between $R$ and $h^{2}$ defined by $a_{n}\left(h^{2}\right)$ and $b_{n}\left(h^{2}\right)$ and plot them. Mathieu did not do it, but Ince did plot it [15], and his figure is given as Figure B.1. Note that Ince's notation from [15] is slightly different than Mathieu's [8]: $a \equiv R$ and $q \equiv-h^{2} / 8$, i.e. his starting equation was:

$$
\frac{d^{2} y}{d x^{2}}+(a+16 q \cos 2 x) y=0
$$

Asymptotic expansions of periodic Mathieu functions valid for large values of $q=h^{2}$, were derived by Dingle and Müller [55].

Appendix C: Expressions for first nine transition curves obtained by harmonic balancing

The expressions for the transition curves of Mathieu's equation (A.8) obtained by harmonic balancing are as follows [1]:

$$
\begin{array}{r}
\delta=-\frac{\epsilon^{2}}{2}+\frac{7 \epsilon^{4}}{32}-\frac{29 \epsilon^{6}}{144}+\frac{68687 \epsilon^{8}}{294912}-\frac{123707 \epsilon^{10}}{409600}+\frac{8022167579 \epsilon^{12}}{19110297600}+\cdots, \\
\quad \delta=\frac{1}{4}-\frac{\epsilon}{2}-\frac{\epsilon^{2}}{8}+\frac{\epsilon^{3}}{32}-\frac{\epsilon^{4}}{384}-\frac{11 \epsilon^{5}}{4608}+\frac{49 \epsilon^{6}}{36864}-\frac{55 \epsilon^{7}}{294912}-\frac{83 \epsilon^{8}}{552960} \\
+\frac{12121 \epsilon^{9}}{117964800}-\frac{114299 \epsilon^{10}}{6370099200}-\frac{192151 \epsilon^{11}}{15288238080}+\frac{83513957 \epsilon^{12}}{8561413324800}+\cdots,
\end{array}
$$




$$
\begin{aligned}
& \delta=\frac{1}{4}+\frac{\epsilon}{2}-\frac{\epsilon^{2}}{8}-\frac{\epsilon^{3}}{32}-\frac{\epsilon^{4}}{384}+\frac{11 \epsilon^{5}}{4608}+\frac{49 \epsilon^{6}}{36864}+\frac{55 \epsilon^{7}}{294912}-\frac{83 \epsilon^{8}}{552960} \\
& -\frac{12121 \epsilon^{9}}{117964800}-\frac{114299 \epsilon^{10}}{6370099200}+\frac{192151 \epsilon^{11}}{15288238080}+\frac{83513957 \epsilon^{12}}{8561413324800}+\cdots \\
& \delta=1-\frac{\epsilon^{2}}{12}+\frac{5 \epsilon^{4}}{3456}-\frac{289 \epsilon^{6}}{4976640}+\frac{21391 \epsilon^{8}}{7166361600} \\
& -\frac{2499767 \epsilon^{10}}{14447384985600}+\frac{1046070973 \epsilon^{12}}{97086427103232000}+\cdots \\
& \delta=1+\frac{5 \epsilon^{2}}{12}-\frac{763 \epsilon^{4}}{3456}+\frac{1002401 \epsilon^{6}}{4976640}-\frac{1669068401 \epsilon^{8}}{7166361600} \\
& +\frac{4363384401463 \epsilon^{10}}{14447384985600}-\frac{40755179450909507 \epsilon^{12}}{97086427103232000}+\cdots, \\
& \delta=\frac{9}{4}+\frac{\epsilon^{2}}{16}-\frac{\epsilon^{3}}{32}+\frac{13 \epsilon^{4}}{5120}+\frac{5 \epsilon^{5}}{2048}-\frac{1961 \epsilon^{6}}{1474560}+\frac{609 \epsilon^{7}}{3276800}+\frac{4957199 \epsilon^{8}}{33030144000} \\
& -\frac{872713 \epsilon^{9}}{8493465600}+\frac{421511 \epsilon^{10}}{23488102400}+\frac{16738435813 \epsilon^{11}}{1331775406080000}-\frac{572669780189 \epsilon^{12}}{58706834227200000}+\cdots, \\
& \delta=\frac{9}{4}+\frac{\epsilon^{2}}{16}+\frac{\epsilon^{3}}{32}+\frac{13 \epsilon^{4}}{5120}-\frac{5 \epsilon^{5}}{2048}-\frac{1961 \epsilon^{6}}{1474560}-\frac{609 \epsilon^{7}}{3276800}+\frac{4957199 \epsilon^{8}}{33030144000} \\
& +\frac{872713 \epsilon^{9}}{8493465600}+\frac{421511 \epsilon^{10}}{23488102400}-\frac{16738435813 \epsilon^{11}}{1331775406080000}-\frac{572669780189 \epsilon^{12}}{58706834227200000}+\cdots, \\
& \delta=4+\frac{\epsilon^{2}}{30}+\frac{433 \epsilon^{4}}{216000}-\frac{5701 \epsilon^{6}}{170100000}-\frac{112236997 \epsilon^{8}}{31352832000000} \\
& +\frac{8417126443 \epsilon^{10}}{123451776000000000}+\frac{2887659548698709 \epsilon^{12}}{265470699110400000000000}+\cdots,
\end{aligned}
$$




$$
\begin{array}{r}
\delta=4+\frac{\epsilon^{2}}{30}-\frac{317 \epsilon^{4}}{216000}+\frac{10049 \epsilon^{6}}{170100000}-\frac{93824197 \epsilon^{8}}{31352832000000} \\
+\frac{21359366443 \epsilon^{10}}{123451776000000000}-\frac{2860119307587541 \epsilon^{12}}{265470699110400000000000}+\cdots
\end{array}
$$

Note that by using the substitutions $\delta=R / 4, \epsilon=-h^{2} / 2$ to correlate Eqs. (A.8) and (A.9), one can match Eqs. (C.2) and (C.3) with Ince's solutions (B.10) and (B.12).

\section{Appendix D: Approximations to the parameters from Section 5.2}

$$
\begin{aligned}
\omega_{1} & =0 \\
\omega_{2} & =-\frac{\delta_{1} \alpha^{2} \beta \tau_{0}^{2}}{8 \omega_{0}} \\
\omega_{3} & =\frac{\delta_{1} \alpha^{2} \beta\left(\beta+\delta_{1}\right) \tau_{0}^{2}}{2 \omega_{0}} \\
\tau_{1} & =-2\left(\beta+\delta_{1}\right) \tau_{0} \\
\tau_{2} & =4\left(\beta+\delta_{1}\right)^{2} \tau_{0} \\
\tau_{3} & =8\left(\beta+\delta_{1}\right)^{3} \tau_{0}-\frac{\alpha^{2}}{12}\left(\beta-2 \delta_{1}\right) \tau_{0}^{3} \\
\tau_{4} & =-16\left(\beta+\delta_{1}\right)^{4} \tau_{0}-8 \beta\left(\beta+\delta_{1}\right)^{3} \tau_{0}^{3}+\frac{\alpha^{2}}{3}\left(\beta+\delta_{1}\right)\left(5 \beta-\delta_{1}\right) \tau_{0}^{3} \\
& -\frac{4 \delta_{1} \alpha^{2} \beta\left(\beta+\delta_{1}\right)^{2}}{\left(2 \beta+2 \delta_{1}-\alpha\right)\left(2 \beta+2 \delta_{1}+\alpha\right)} \tau_{0}^{3}
\end{aligned}
$$

Appendix E: Hill's determinants obtained for Mathieu's equation with elliptic-type excitation

Hill's determinants for the Case I and II obtained for Mathieu's equation with elliptic-type excitation by harmonic balancing in Section 8 are: 


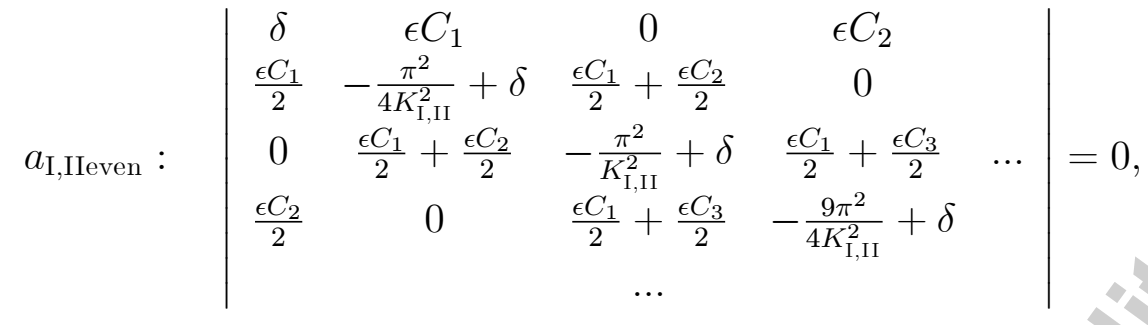

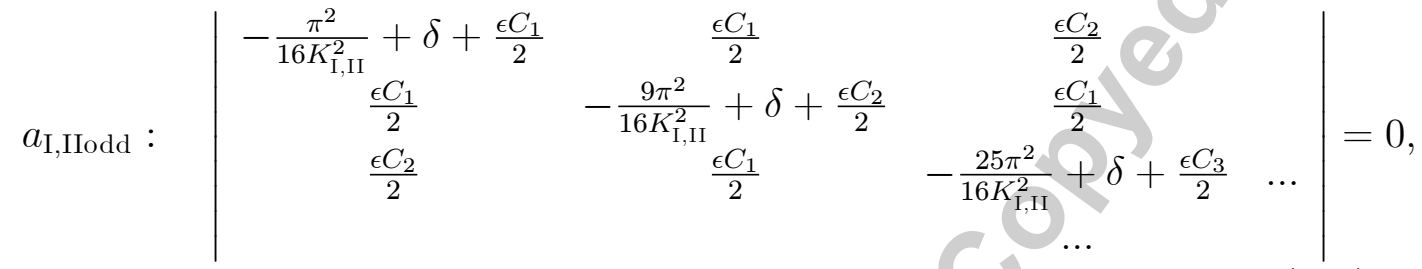

$$
\begin{aligned}
& b_{\mathrm{I}, \mathrm{IIeven}}:\left|\begin{array}{cccc}
-\frac{\pi^{2}}{4 K_{\mathrm{I}, \mathrm{II}}^{2}}+\delta & \frac{\epsilon C_{1}}{2}-\frac{\epsilon C_{2}}{2} & 0 \\
\frac{\epsilon C_{1}}{2}-\frac{\epsilon C_{2}}{2} & -\frac{\pi^{2}}{K_{\mathrm{I}, \mathrm{II}}^{2}}+\delta & \frac{\epsilon C_{1}}{2}-\frac{\epsilon C_{3}}{2} & \\
0 & \frac{\epsilon C_{1}}{2}-\frac{\epsilon C_{3}}{2} & -\frac{9 \pi^{2}}{4 K_{\mathrm{I}, \mathrm{II}}^{2}}+\delta & \ldots
\end{array}\right|=0 \text {, }
\end{aligned}
$$

For Case III considered in Section 3.1, Hill's determinants are

$$
a_{\mathrm{IIIeven}}: \mid \begin{array}{cccc}
\delta+\epsilon D_{0} & \epsilon D_{1} & \epsilon D_{2} & \epsilon D_{3} \\
\frac{\epsilon D_{1}}{2} & -\frac{m_{\mathrm{III}} \pi^{2}}{K_{\mathrm{III}}^{2}}+\delta & \frac{\epsilon D_{1}}{2}+\frac{\epsilon D_{3}}{2} & \frac{\epsilon D_{2}}{2}+\frac{\epsilon D_{4}}{2} \\
& +\epsilon D_{0}+\frac{\epsilon D_{2}}{2} & \frac{4 m_{\mathrm{III}} \pi^{2}}{K_{\mathrm{III}}}+\delta & \frac{\epsilon D_{1}}{2}+\frac{\epsilon D_{5}}{2}
\end{array} \quad \ldots \quad=0 \text {, }
$$




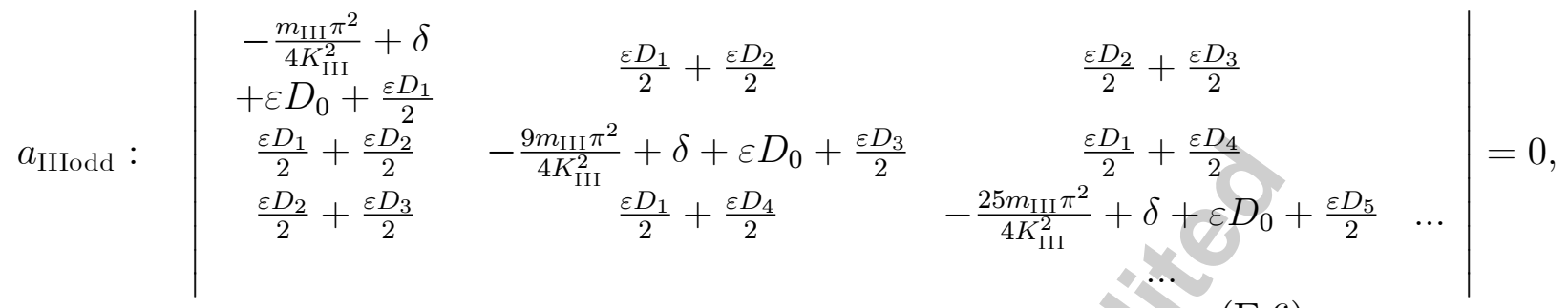

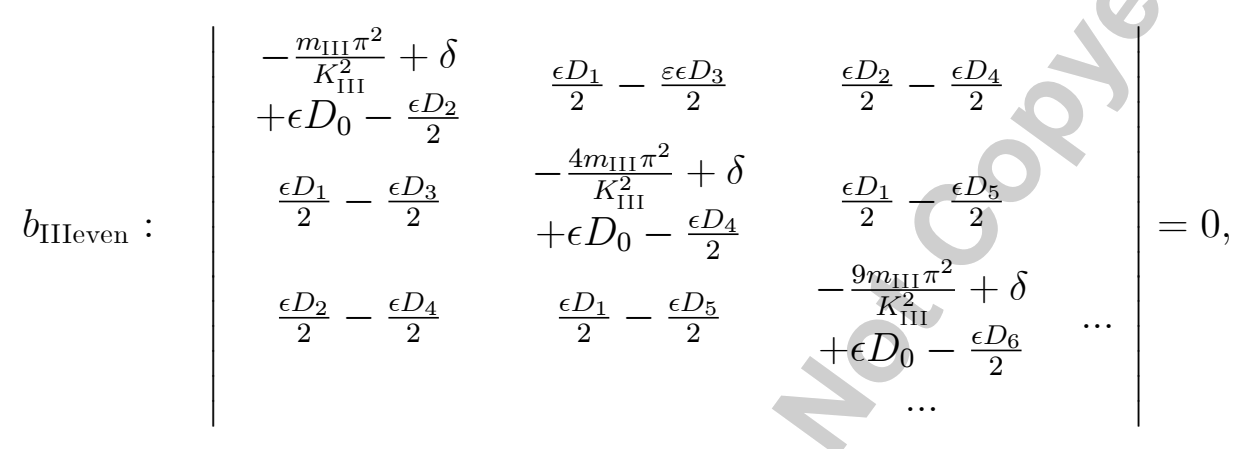

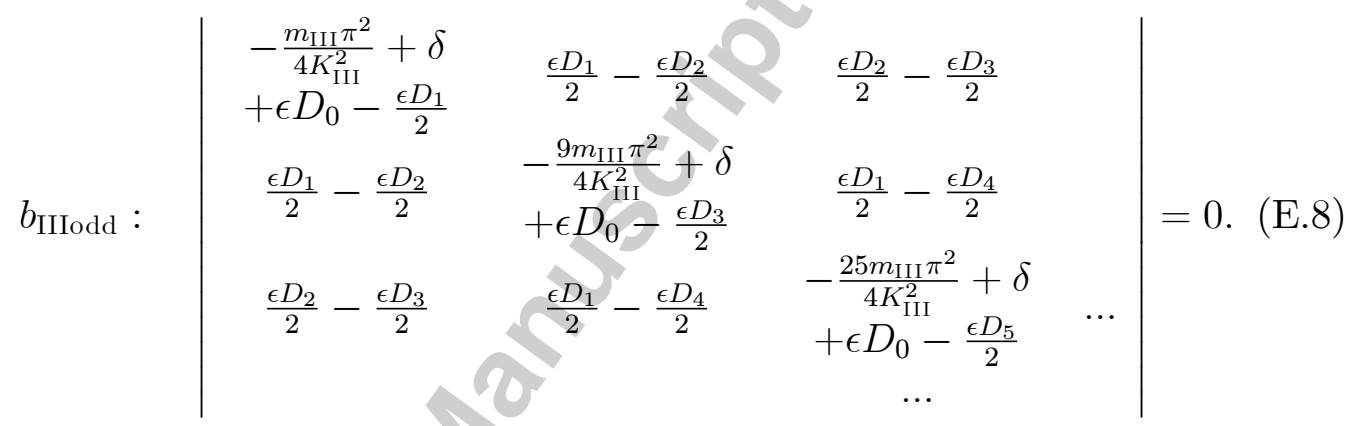

\section{References}

[1] R.H. Rand, Lecture Notes on Nonlinear Vibrations (version 53), http://dspace.library.cornell.edu/handle/1813/28989, Accessed 23 July 2017.

[2] W. Magnus, S. Winkler, Research Report No. BR-38: Hill's Equation, Part II: Transformations, Approximation, Examples, New York University, New York, 1961. 
[3] N.W. McLachlan, Theory and Applications of Mathieu Functions, Clarendon Press, Oxford, 1947.

[4] A. Erdelyi, Higher Transcendental Functions, Volume III, McGraw-Hill Book Company, New York, 1955, re-issued 1981.

[5] J.J. Stoker, Nonlinear Vibrations in Mechanical and Electrical Systems, Interscience Publishers, New York, 1950.

[6] M. Cartmell, Introduction to Linear, Parametric and Nonlinear Vibrations, Chapman and Hall, London, 1990.

[7] L. Ruby, Applications of the Mathieu equation, American Journal of Physics, Vol. 64, pp. 39-44, 1996.

[8] E. Mathieu, Mémoire sur Le Mouvement Vibratoire d'une Membrane de forme Elliptique. Journal de Mathématiques Pures et Appliquées, Vol. 13, pp. 137-203, 1868.

[9] E. Heine, Hanbuch der Kugelfunktionen, 2 Vols, 1878/81.

[10] G. Floquet, Sur les equations differetielles lineaires, Ann. de l' cole Normale Superieure, Vol. 12, pp. 47-88, 1883.

[11] G.W. Hill, Mean motion of the lunar perigee, Acta Mathematica, Vol. 8, pp. 1-36, 1886.

[12] Lord Rayleigh, Maintenance of vibrations by forces of double frequency and propagation of waves through a medium with a periodic structure, P.M. Vol. 24, pp. 145-159, 1887.

[13] B. Sieger, Die Beugung einer ebened elektrischen Weilen an einem Schirm von elliptischen Querschnitt, A. der P., Vol. 27, pp. 626, 1908.

[14] E.T. Whittaker, Elliptic cylinder functions in harmonic analysis, P.I.C.M., Cambridge, Vol. 1, pp. 366, 1912.

[15] E. Ince, Research into the characteristic numbers of Mathieu equation, P.R.S.E, Vol. 46, pp. 20-29, 1927.

[16] M.J.O. Strutt, Zur Wellenmechanik des Atomgitters, Annalen der Physik, Vol. 391, pp. 319-324, 1928. 
[17] A. Stephenson, On a new type of dynamic stability, Memoirs and Proceedings of the Manchester Literary and Philosophical Society, Vol. 52, pp. 1-10, 1908.

[18] A. Stephenson, On induced stability, Philosophical Magazine, Vol. 15, pp. 233-236, 1908.

[19] J.J. Thomsen, Vibrations and Stability: Advanced Theory, Analysis, and Tools, Springer-Verlag, Berlin, 2003.

[20] A.P. Seyranian, A.A.Mailybaev, Multiparameter stability theory with mechanical applications, World Scientific, series A, Vol. 13, 2003.

[21] F. Verhulst, Perturbation Analysis of Parametric Resonance, www.staff.science.uu.nl/ verhu101/Parametric.pdf, Accessed 5 April 2017.

[22] N. Vlajic, X. Liu, H. Karki, B. Balachandran, Torsional oscillations of a rotor with continuous stator contact, International Journal of Mechanical Sciences, Vol. 83, pp. 65-75, 2014.

[23] T.L Yang, R.M. Rosenberg, On the vibrations of a particle in the plane, International Journal of Non-linear Mechanics, Vol. 2, pp. 1-25, 1967.

[24] T.L Yang, R.M. Rosenberg, On the forced vibrations of a particle in the plane, International Journal of Non-linear Mechanics, Vol. 3, pp. 47-63, 1968.

[25] J.D. Cole, Perturbation Methods in Applied Mathematics, Blaisdell, 1968.

[26] A. Nayfeh, Perturbation Methods, Wiley, 1973.

[27] T. Insperger, G. Stépán, Semi Discretization for Time Delay Systems: Stability and Engineering Applications, Applied Mathematical Science 178, Springer Science+Business Media, 2011.

[28] I. Kovacic I., R. Rand, Duffing-type oscillators with amplitudeindependent period. In: Applied Nonlinear Dynamical Systems (Ed. J. Awrejcewicz), Springer Proceedings in Mathematics \& Statistics, Vol. 93, pp. 1-10, 2014. 
[29] I. Kovacic, M.J. Brennan, The Duffing Equation: Nonlinear Oscillators and their Behaviour, John Wiley and Sons, Chichester, 2011.

[30] R.H. Rand, D.V. Ramani, W.L. Keith, K.M. Cipolla, The quadratically damped Mathieu equation and its application to submarine dynamics. In: Control of Noise and Vibration: New Millenium, AD-Vol. 61, November 5-10, 2000, Orlando, FL, ASME, New York, November 2000, pp. $39-50$.

[31] D.V. Ramani, W.L. Keith, R.H. Rand, Perturbation solution for secondary bifurcation in the quadratically-damped Mathieu equation, International Journal of Non-Linear Mechanics, Vol. 39, pp. 491-502, 2004.

[32] T.M. Morrison, R.H. Rand, 2:1 Resonance in the delayed nonlinear Mathieu equation, Nonlinear Dynamics, Vol. 50, pp. 341-352, 2007.

[33] T. Insperger, G. Stepan, Stability chart for the delayed Mathieu equation, Proceedings of the Royal Society A: Mathematical, Physical and Engineering Sciences, Vol. 458, No. 2024, pp. 1989-1998, 2002.

[34] E.A. Butcher, B.P. Mann, Stability Analysis and Control of Linear Periodic Delayed Systems using Chebyshev and Temporal Finite Element Methods. In: Delay Differential Equations: Recent Advances and New Directions, Eds. B. Balachandran, D. Gilsinn, and T. Kalmar-Nagy, Springer, New York, 2009.

[35] F.M. Atay, Van der Pol's oscillator under delayed feedback, Journal of Sound and Vibration, Vol. 218, pp. 333-339, 1998.

[36] S. Wirkus, R.H. Rand, The dynamics of two coupled van der Pol oscillators with delay coupling, Nonlinear Dynamics, Vol. 30, pp. 205-221, 2002.

[37] S.M. Sah, R. Rand, Delay terms in the slow flow, Journal of Applied Nonlinear Dynamics, Vol. 5, pp. 473-486, 2016.

[38] A. Bernstein, R. Rand, Delay-coupled Mathieu equations in synchrotron dynamics, Journal of Applied Nonlinear Dynamics, Vol. 5, pp. 337-348, 2016. 
[39] R.H. Rand, S.M. Sah, M.K. Suchorsky, Fractional Mathieu equation, Communications in Nonlinear Science and Numerical Simulation, Vol. 15, pp. 3254-3262, 2010.

[40] B. Ross, A brief history and exposition of the fundamental theory of fractional calculus. In: Fractional calculus and its applications, Springer Lecture Notes in Mathematics, Vol. 57, pp. 1-36, 1975.

[41] A. Mesbahi, M. Haeri, M. Nazari, E.A. Butcher, Fractional delayed damped Mathieu equation, International Journal of Control, Vol. 88, pp. 622-630, 2015.

[42] R. Rand, R. Zounes, R. Hastings, Dynamics of a quasiperiodically forced Mathieu oscillator. In: Nonlinear Dynamics: The Richard Rand 50th Anniversary Volume (Ed. A.Guran), World Scientific Pub.Co. pp.2 03$221,1997$.

[43] R. Rand,T. Morrison, 2:1:1 Resonance in the quasi-periodic Mathieu equation, Nonlinear Dynamics, Vol. 40, pp. 195-203, 2005.

[44] R.S. Zounes, R.H.Rand, Transition curves in the quasiperiodic Mathieu equation, SIAM Journal of Applied Mathematics, Vol. 58, pp. 10941115, 1998.

[45] N. Abouhazim, R.H. Rand, M. Belhaq, The damped nonlinear quasiperiodic Mathieu equation near 2:2:1 resonance, Nonlinear Dynamics, Vol. 45, pp. 237-247, 2006.

[46] R. Rand, K. Guennoun, M. Belhaq, 2:2:1 resonance in the quasiperiodic Mathieu equation, Nonlinear Dynamics, Vol. 31, pp. 367-374, 2003.

[47] A.Sharma, S.C.Sinha, An approximate analysis of quasi-periodic systems via Floquet theory, Journal of Computational and Nonlinear Dynamics, Vol. 13, Paper No: CND-17-1104 (18 pages), 2017.

[48] R.S. Zounes, R.H.Rand, Global behavior of a nonlinear quasiperiodic Mathieu equation, Nonlinear Dynamics, Vol. 27, pp. 87-105, 2002.

[49] M. Abramowitz, I. Stegun, Handbook of Mathematical Functions, Dover Publications, New York, 1965. 
[50] I. Kovacic, L. Cveticanin, M. Zukovic, Z. Rakaric, Jacobi elliptic functions: a review of nonlinear oscillatory application problems, Journal of Sound and Vibration, Vol. 380, pp. 1-36, 2016.

[51] P. Byrd, M. Friedman, Handbook of Elliptic Integrals for Engineers and Scientists, Springer, Berlin, 1954.

[52] I.S. Gradshteyn, I.M. Ryzhik, Tables of Integrals, Series and Products, Academic Press, New York, 2000.

[53] I. Kovacic, M. Zukovic, A pendulum with an elliptic-type parametric excitation: stability charts for a damped and undamped system, Communications in Nonlinear Science and Numerical Simulation, Vol. 19, pp. 1185-1202, 2014.

[54] S.M. Sah, B. Mann, Transition curves in a parametrically excited pendulum with a force of elliptic type, Proceedings of the Royal Society A: Mathematical, Physical and Engineering Sciences, Vol. 468, pp. 39954007, 2012.

[55] R.B. Dingle, H.J.W. Müller-Kirsten, Asymptotic expansions of Mathieu functions and their characteristic numbers, Journal für die reine und angewandte Mathematik, Vol. 21, pp. 11-32, 1962. 


\section{FIGURE CAPTIONS:}

Figure 1. a) Mathematical pendulum whose support moves periodically in a vertical direction; b) "The particle in the plane" problem.

Figure 2. a) Two transition curves of Mathieu's equation for the first region of instability called a tongue for an undamped case (solid line), Eq. (25); b) Example of motion of Point P1 located inside the tongue; c) Example of motion of Point P2 located outside the tongue.

Figure 3. A stability chart of classical Mathieu's equation (1) obtained by using numerical integration in conjunction with Floquet theory: grey region $=$ unstable $(\mathrm{U})$, white region $=$ stable $(\mathrm{S})$. Red dotted line-transition curves obtained by harmonic balancing, Eqs. (C.1)-(C.9).

Figure 4. a) Analytically obtained transition curves of Mathieu's equation for the first tongue for an undamped case (solid line), Eq. (25) and the case with linear viscous damping (dashed line), Eq. (52); b) Numerically obtained stability chart of damped of Mathieu's equation, Eq. (45).

Figure 5 . The $\delta-\epsilon$ plane of Mathieu's equation with cubic geometric nonlinearity, Eq. (56): a) existence of different equilibria; b) bifurcations.

Figure 6. Phase portraits of the Poincaré map in the different regions of the parameter plane in a quadratically damped Mathieu equation, Eq. (66).

Figure 7. a) First instability tongue in the parameter space $(\delta, \tau, \epsilon)$, Eq. (99), for $\beta=3 / 5, \alpha=1, \mu=0$; b) Three-dimensional stability chart in the parameter space $(\delta, b, \epsilon)$ of the delayed undamped Mathieu equation with $\tau=2 \pi$, redrawn based on [33] (adapted from [32] with permission from The Royal Society).

Figure 8. Hopf bifurcation curye, Eq. (106), for $\epsilon=0.05, \beta=3 / 5, \alpha=1$. $\mathrm{LC}=$ limit cycle, No LC $=$ no limit cycle.

Figure 9 First transition curves, Eq. (119) in the fractional Mathieu equation, Eq. (109) for $c=0.1$ and different values of $\alpha$.

Figure 10. Plot of the minimum quantity of forcing amplitude $\epsilon_{\min } / c$, Eq. (120) necessary to produce instability as a function of fractional derivative order $\alpha$.

Figure 11. First transition curves, Eq. (123) for the Mathieu equation with two fractional terms and delay, Eq. (122) for $b=c=0.1$, different values of $\tau, \Lambda^{*}$ satisfying Eq. (124) and: a) $\alpha=0.25$; b) $\alpha=0.75$.

Figure 12. As a first approximation we may think of the instabilities occurring in Eq. (125) as consisting of the union of the instabilities in Eqs. (126) and (127). 
Figure 13. Same information as in Figure 12 plotted for fixed $\epsilon$ with varying $\omega$ and $\delta$.

Figure 14. a) Stability of Eq. (125) as determined directly from numerical integration. $\epsilon=0.1$; b) Enlargement of Figure 14a around $\delta=0.25, \omega=1$. $\epsilon=0.1$; c) Enlargement of Figure 14a around $\delta=0.25, \omega=0.1 . \quad \epsilon=0.1$. Black $=$ stable, White $=$ unstable.

Figure 15. Stability of Eq. (125) as determined directly from numerical integration: a) $\epsilon=0.01$;

$\mathrm{b}=\epsilon=0.05$; c) $\epsilon=0.1$; d) $\epsilon=0.5$ (Note that Figures $15 \mathrm{a}-\mathrm{c}$ lie in the region bounded by the thick straight lines in this part); e) $\epsilon=1$. Black $=$ stable, White $=$ unstable.

Figure 16. Period of the elliptic-type cn excitation as a function of the elliptic parameter $m$ for Cases I-III.

Figure 17. Stability charts of Eq. (129), where gray regions indicate instability: a) $m=-0.5$; b) $m=0.5$; c) $m=1.5$. Results obtained by means of the approximated equation of motion are labelled by red solid lines (in all cases $\tilde{N}=10$ ).

Figure B.1. Stability chart redrawn based on a figure from page 28 of Ince's paper [15].

Animation 1 (see Supplementary Material to this article). Stability charts of Eq. (129) for different values of the elliptic parameter $m$. 
Figure 1

a)

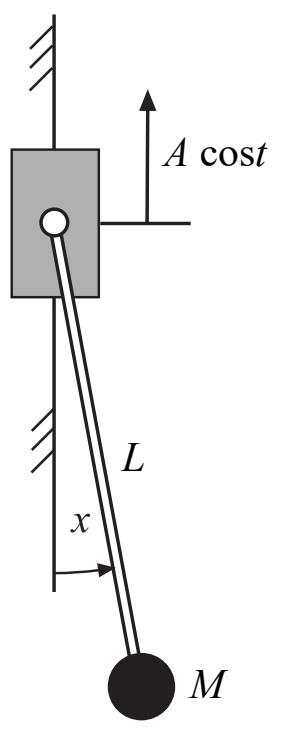

b)

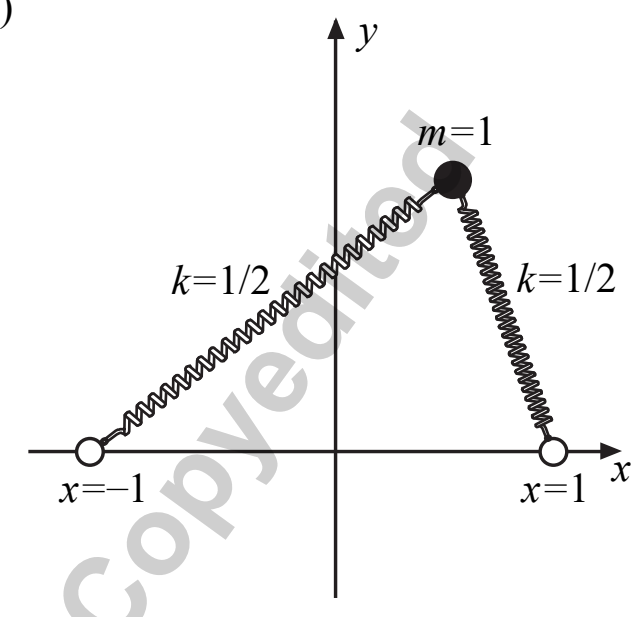


Figure 2

a)

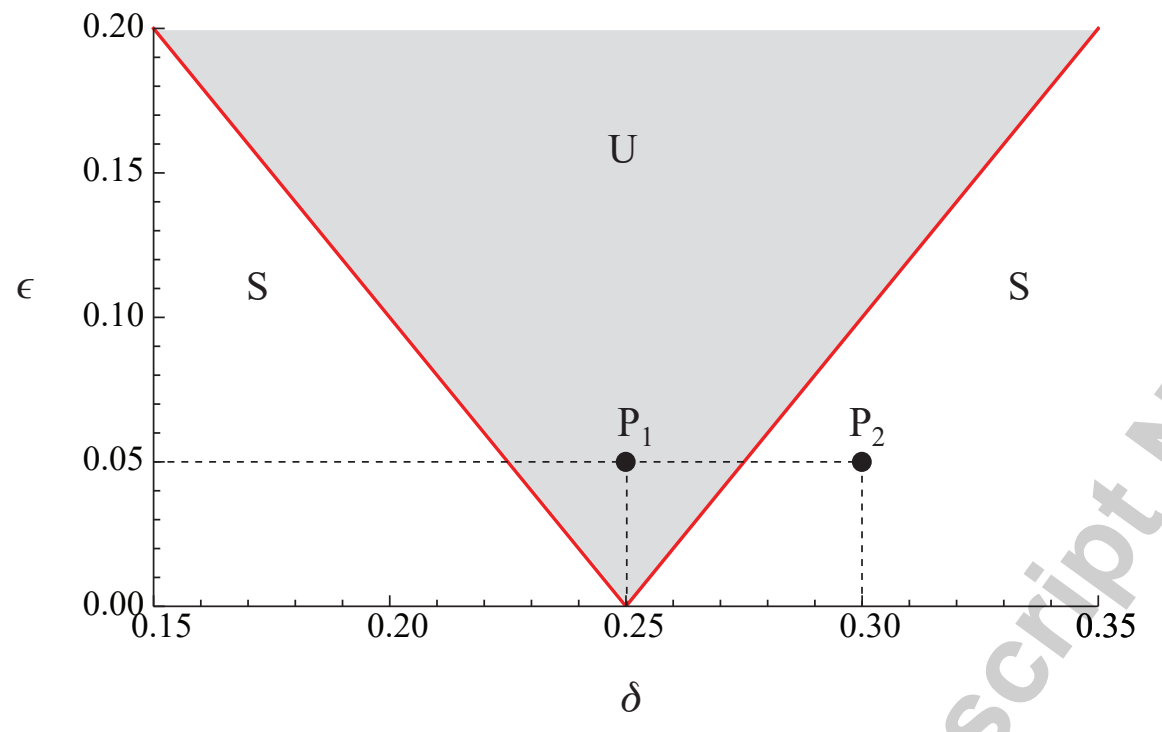

b)

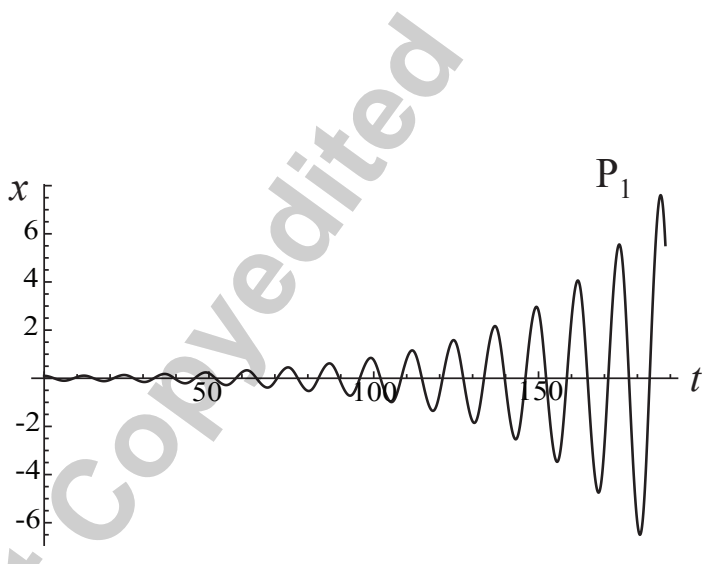

c)

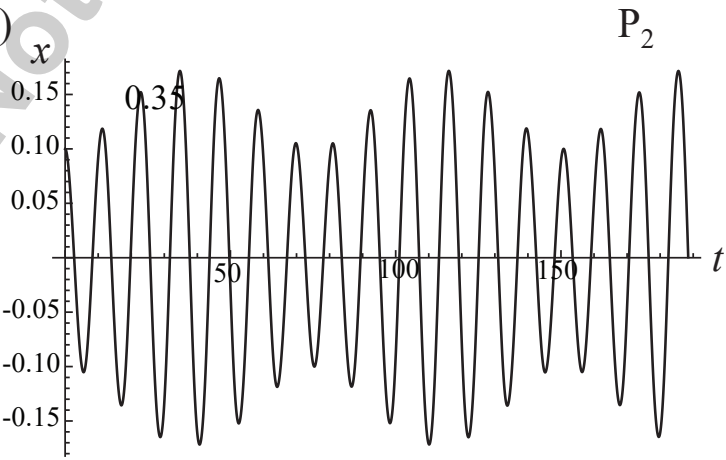


Figure 3

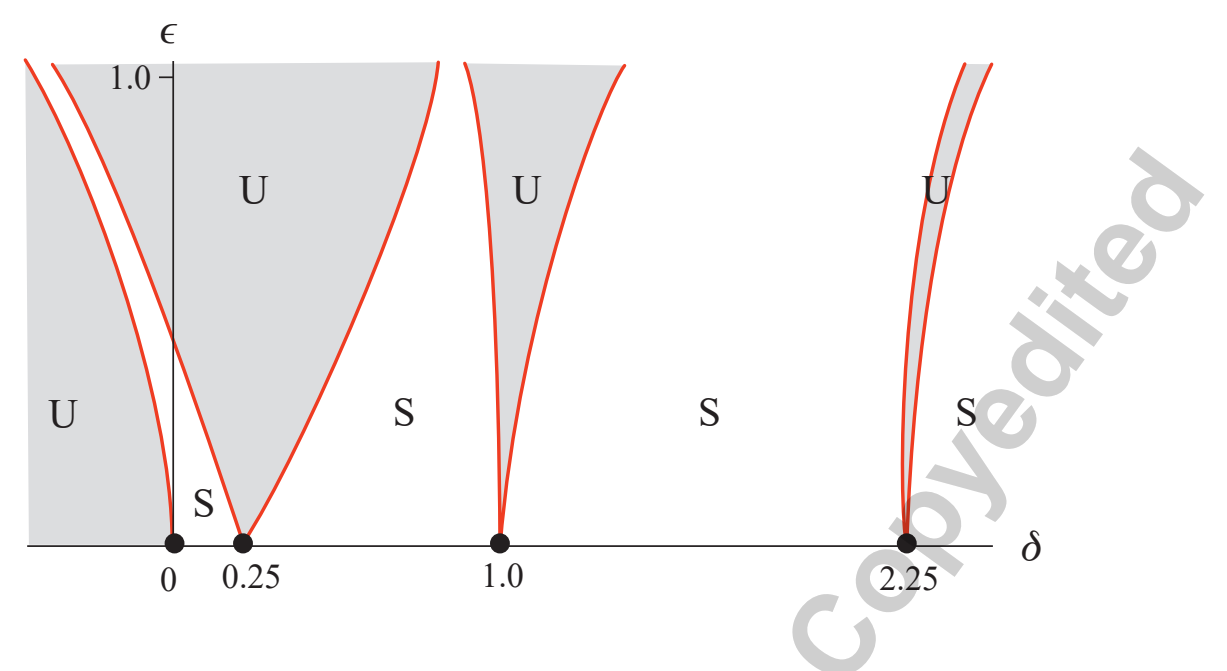


Applied Mechanics Reviews. Received AFigure-42017;

Acceajed manuscript posted January 31, 2018. doi:10.1115/1.4039144

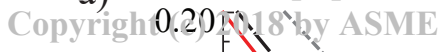

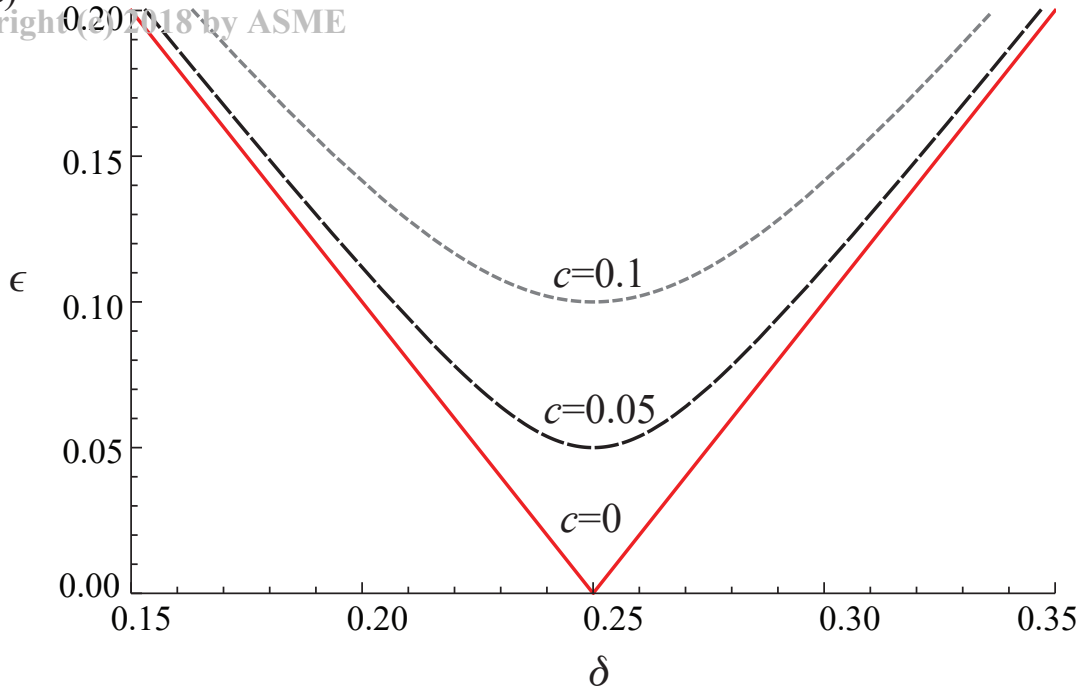

b)

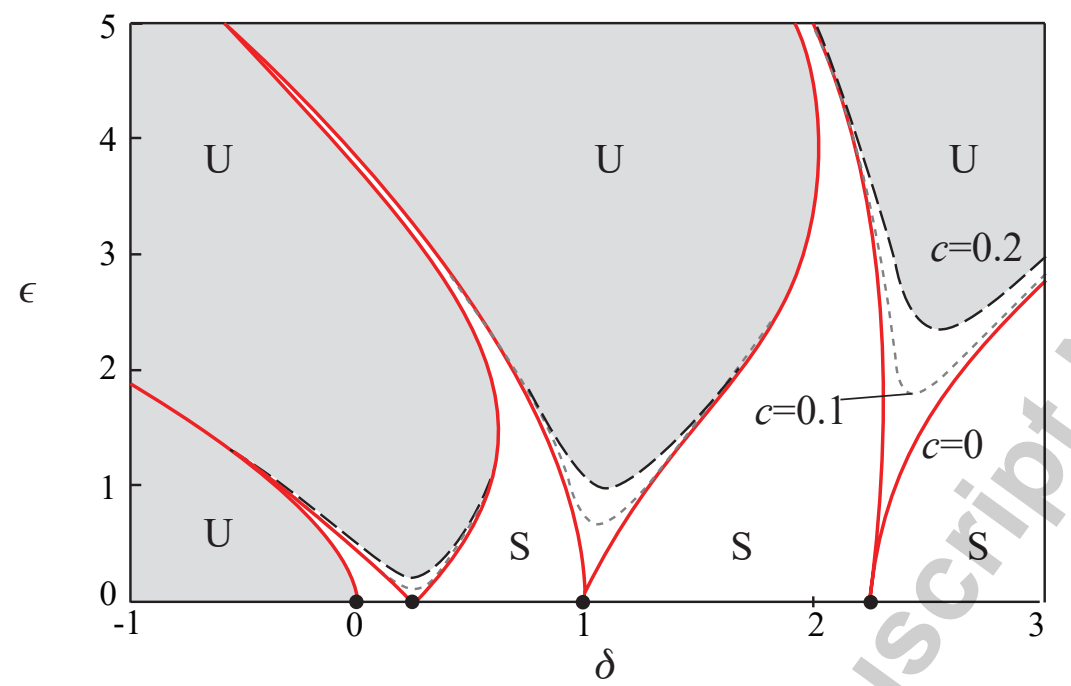


Figure 5

a)

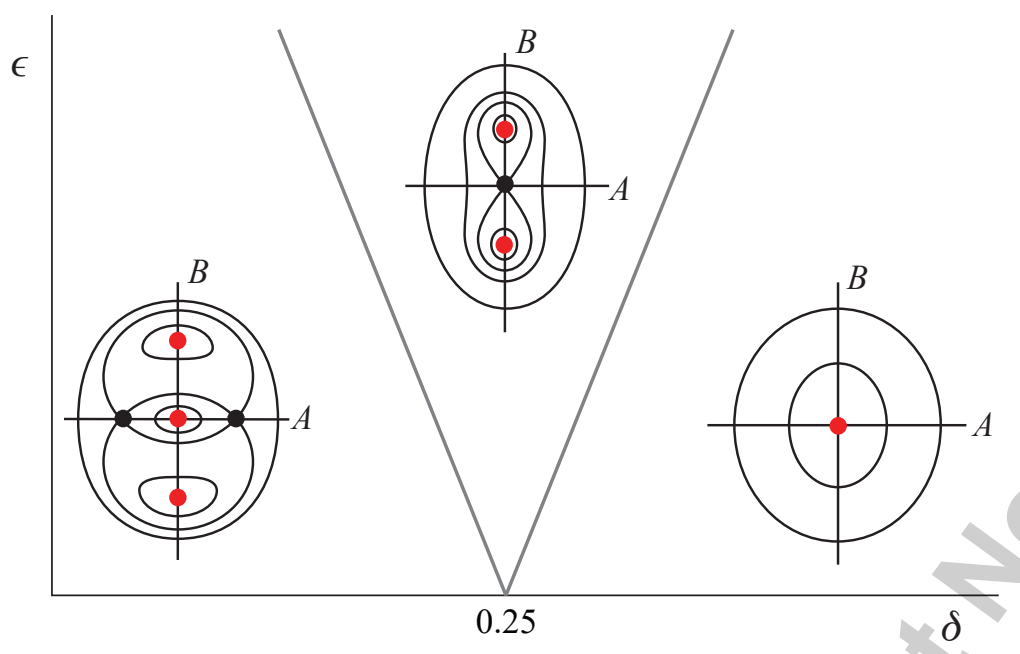

b)

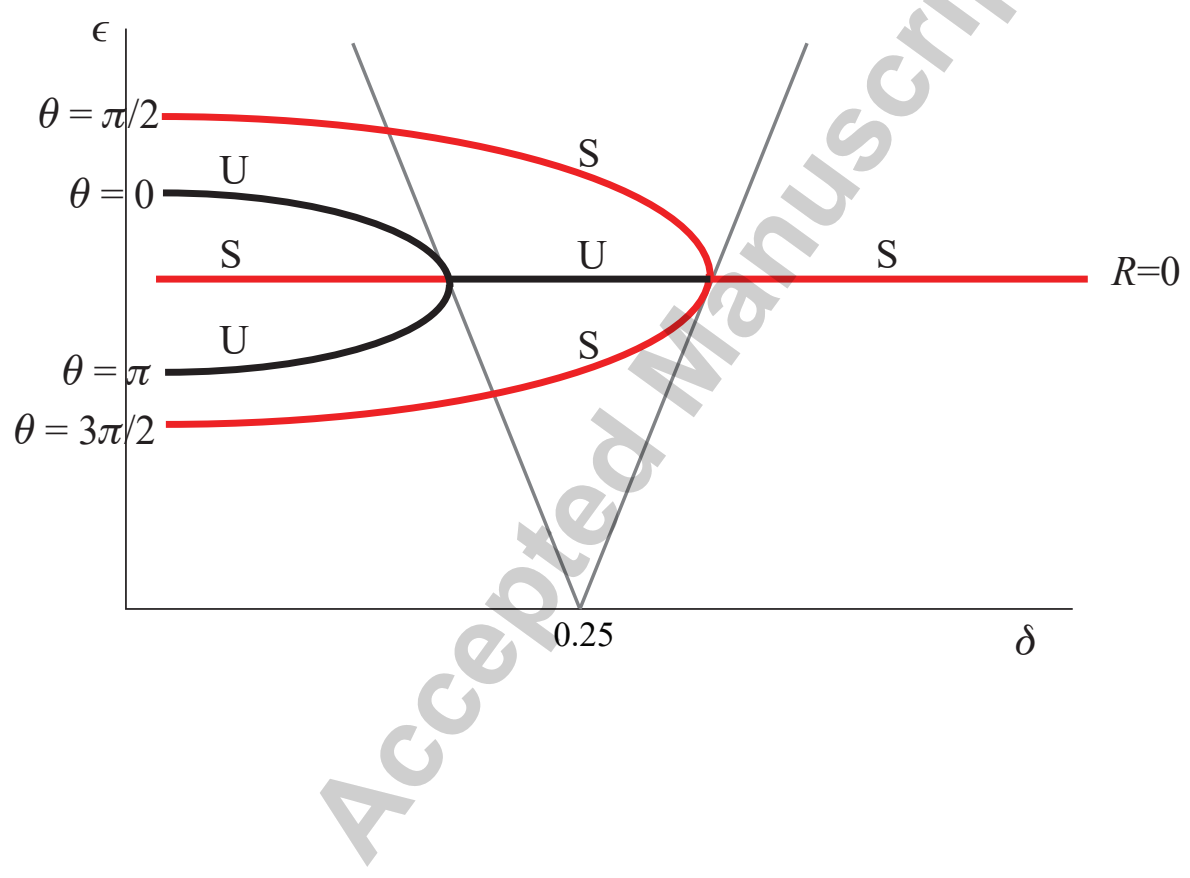


Figure 6

Applied 3.6 echanics Reviews. Received Auoust 07, 2017:

Accepted in anuscript posted Jant?

Copyright (c) 2018 by ASMIE
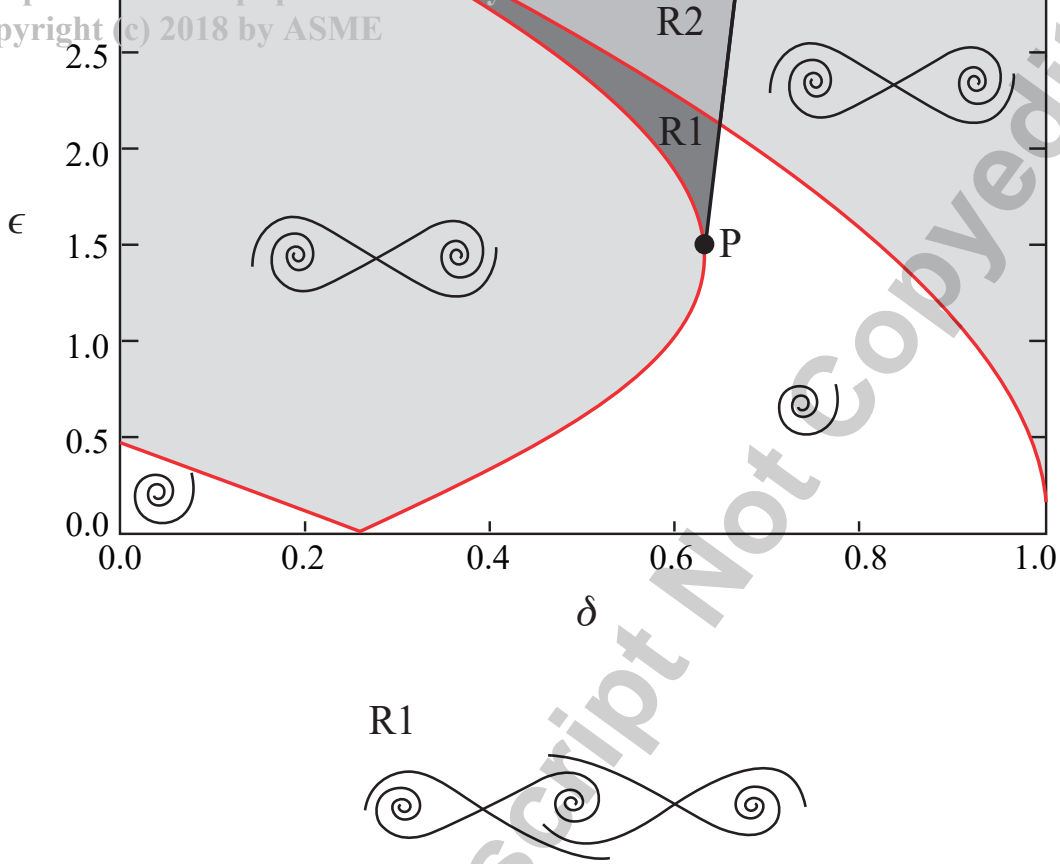

$\mathrm{R} 2$

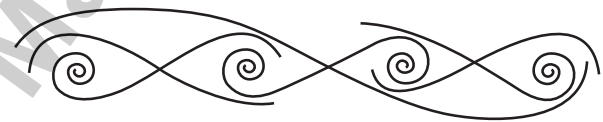

Downloaded From: http://appliedmechanicsreviews.asmedigitalcollection.asme.org/ on 02/13/2018 Terr 
Figure 7

a)

b)
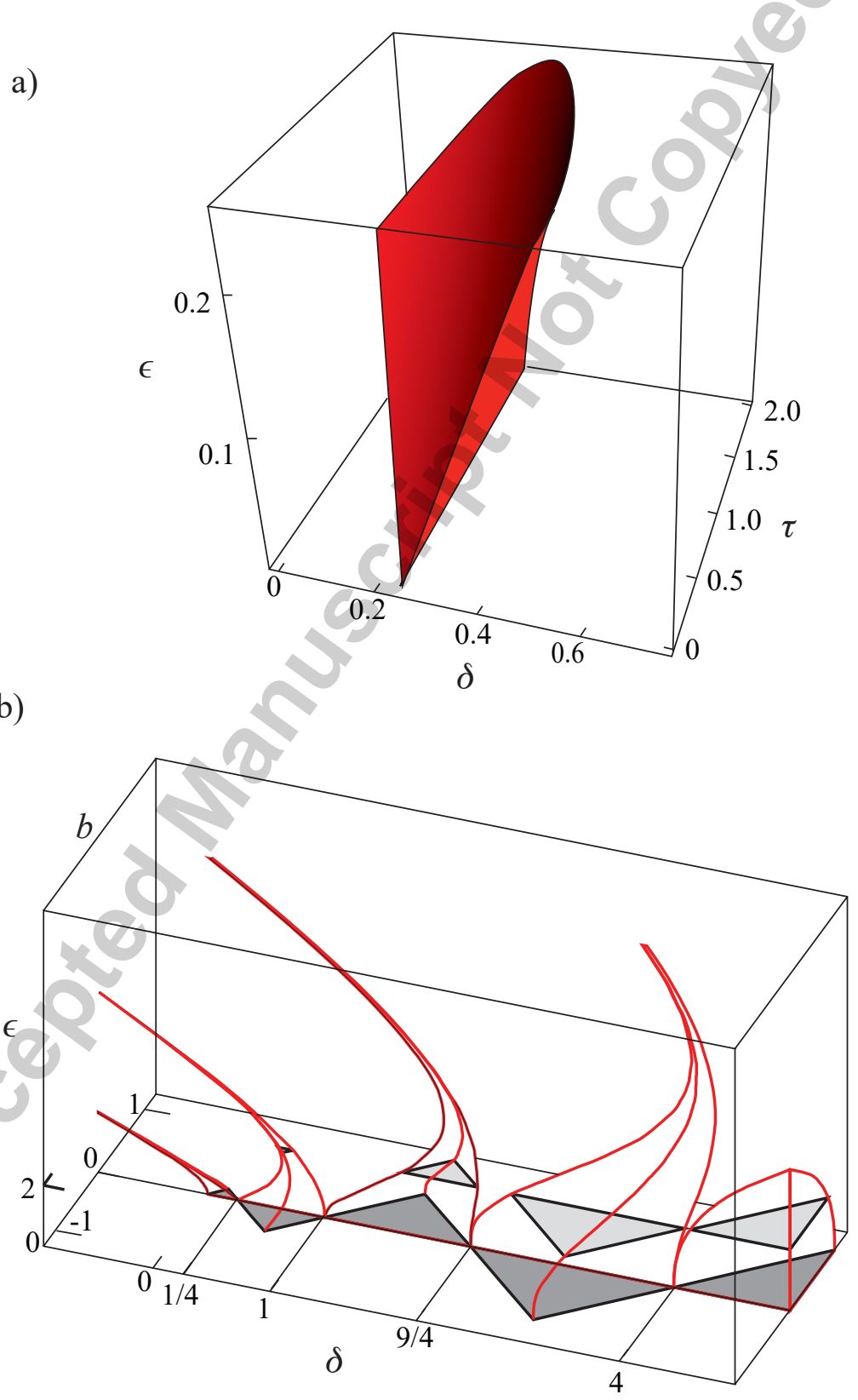


\section{Figure 8}

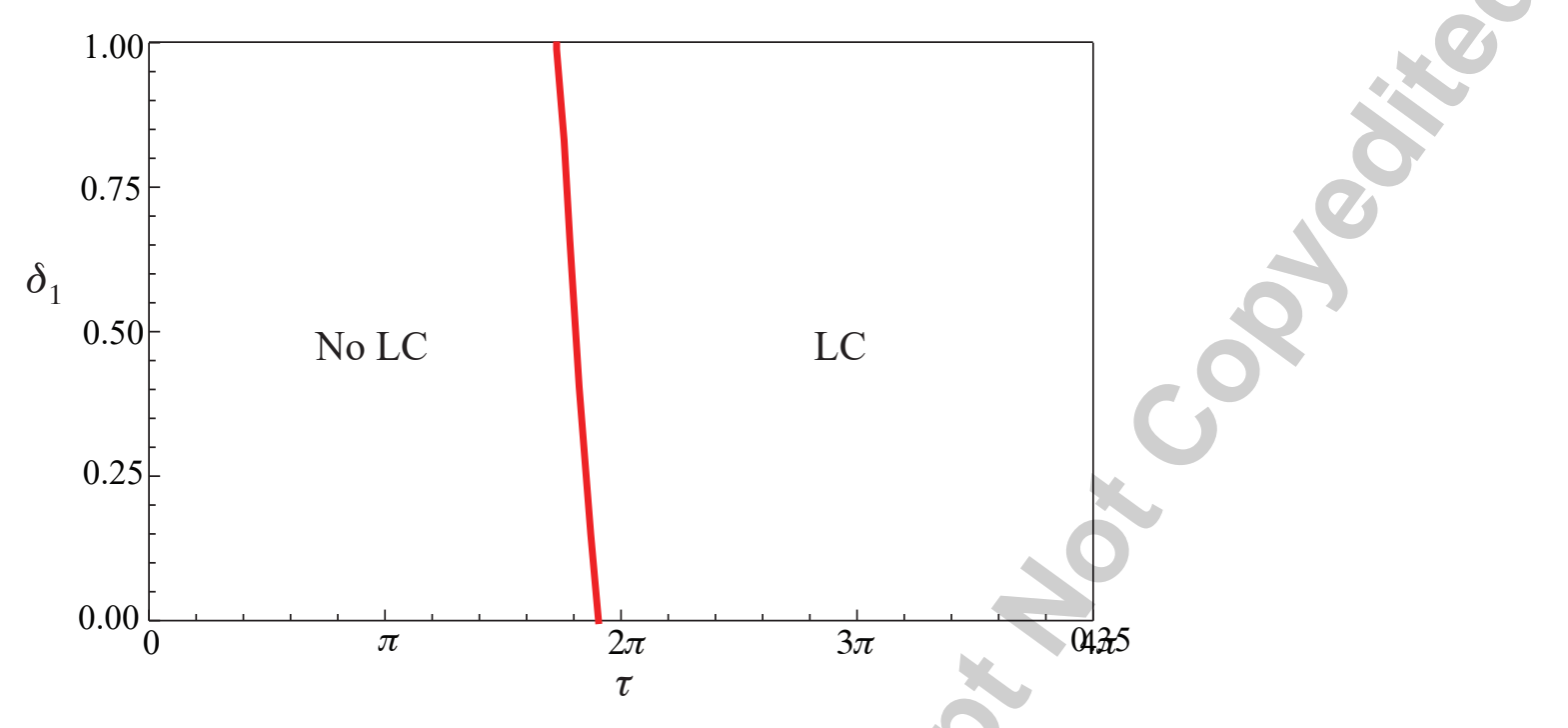


Figure 9

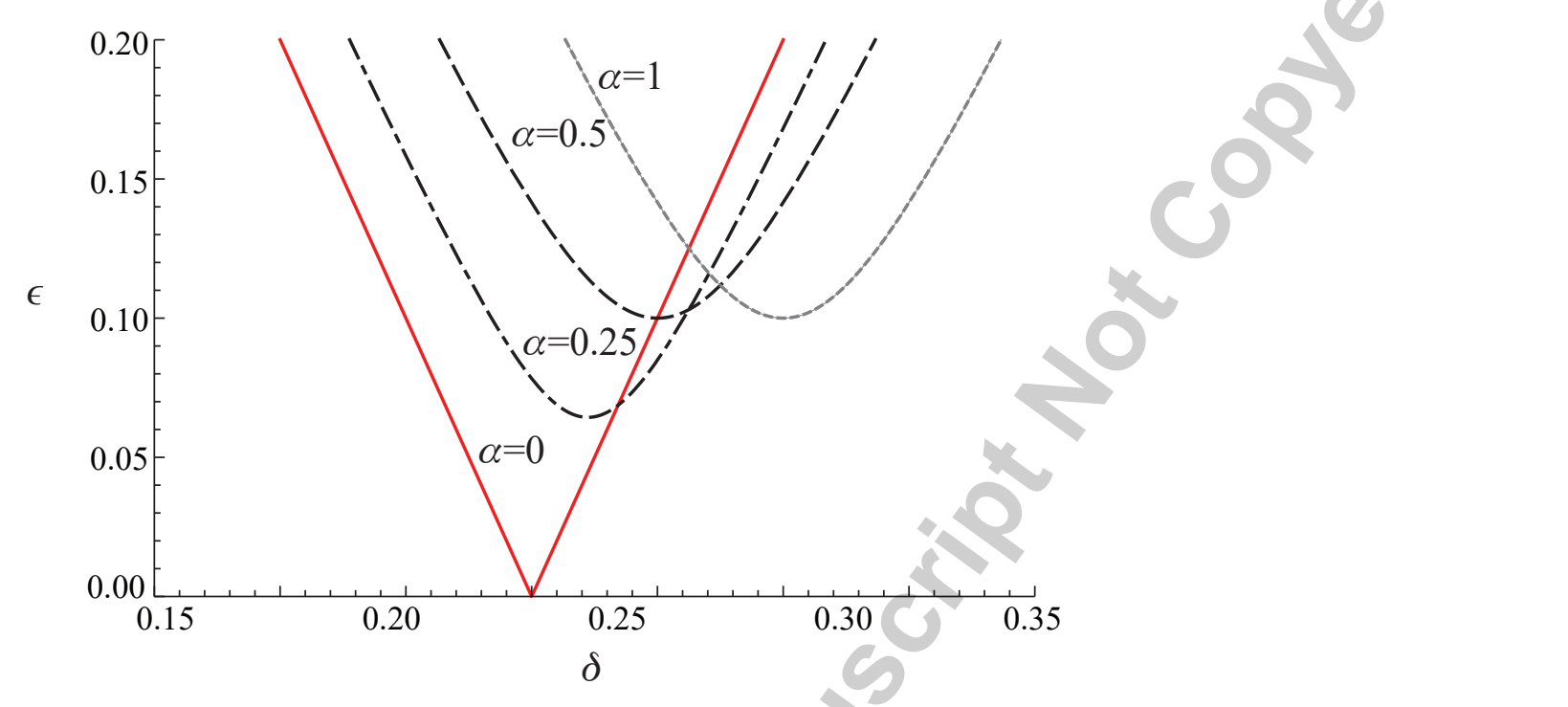


Figure 10

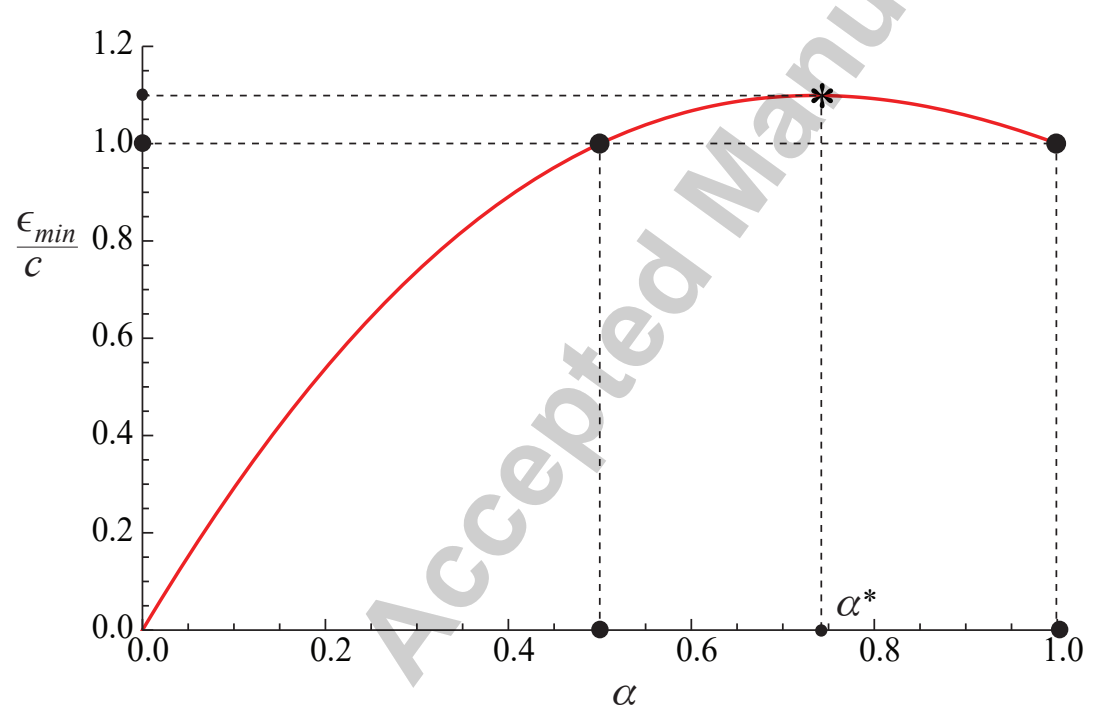


Figure 11

a)

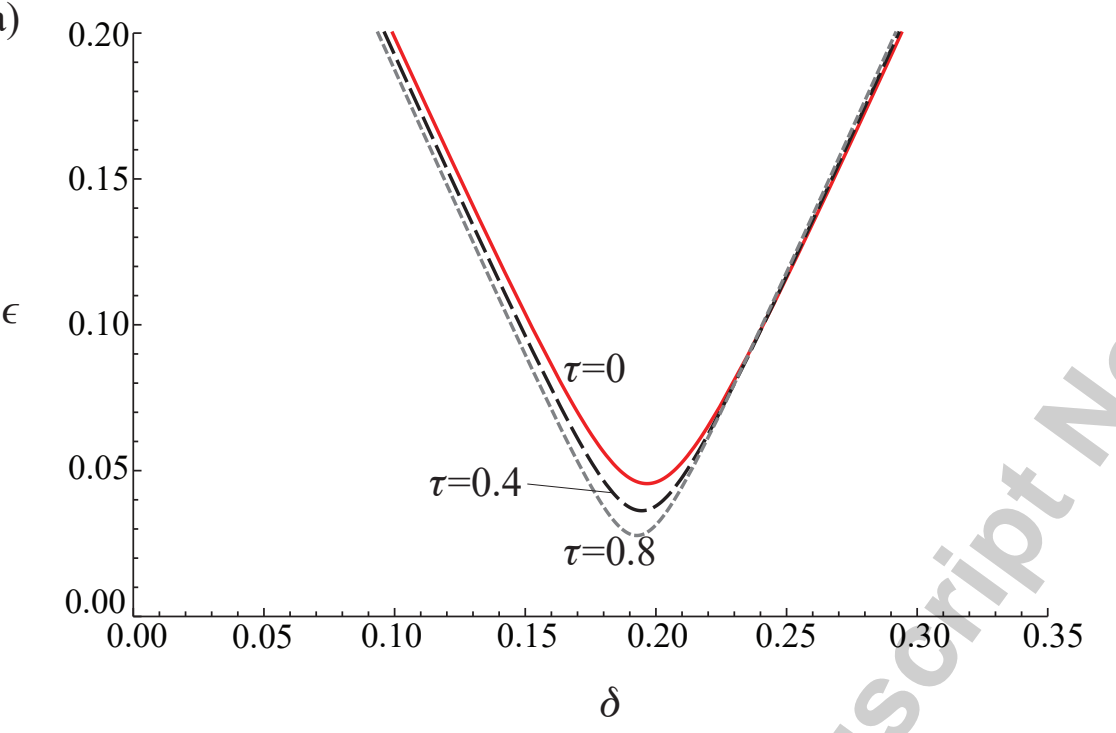

b)

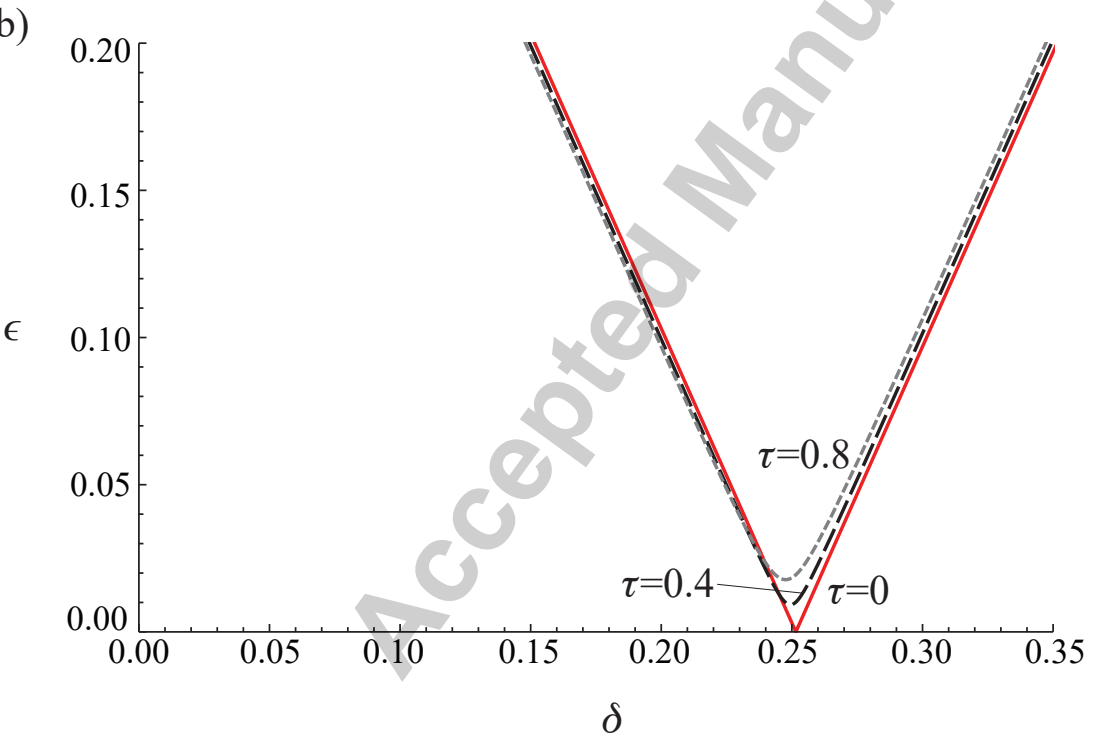


Figure 12

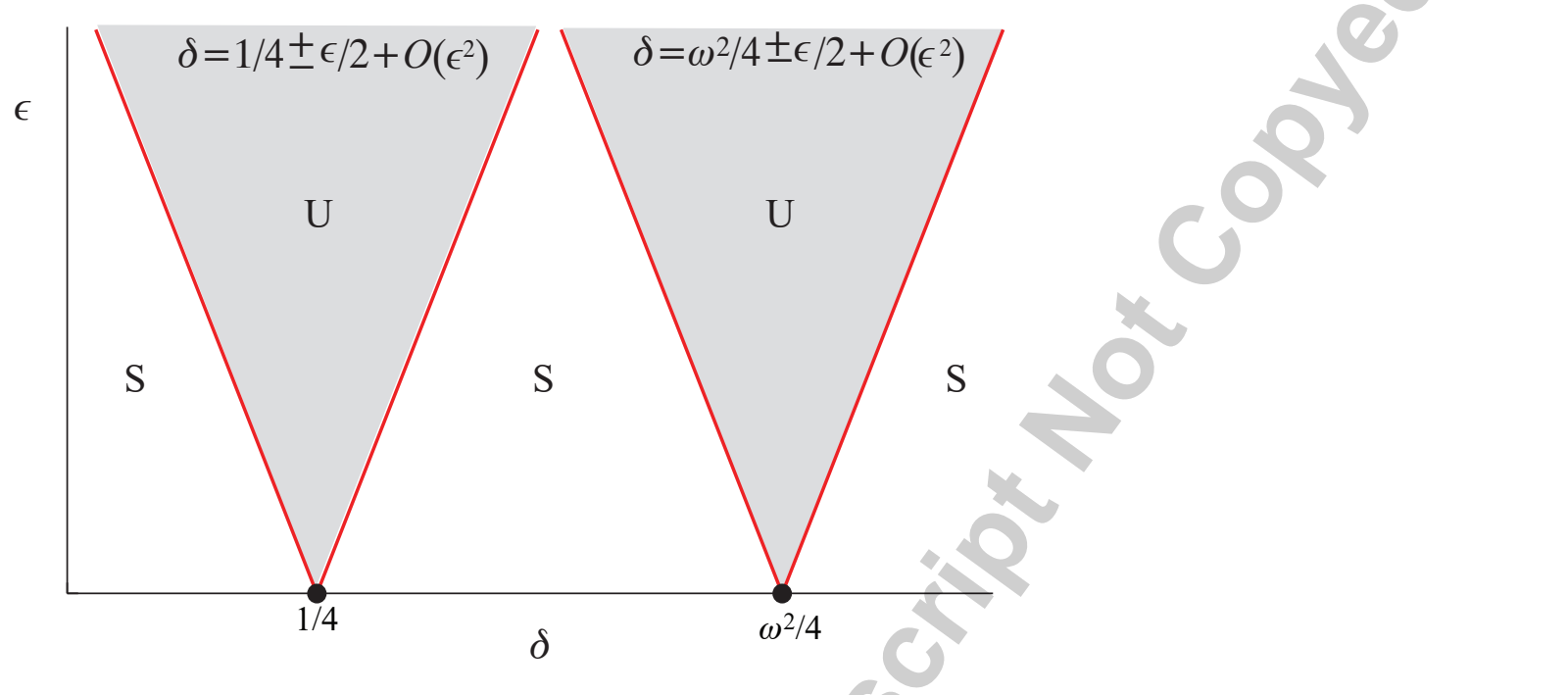




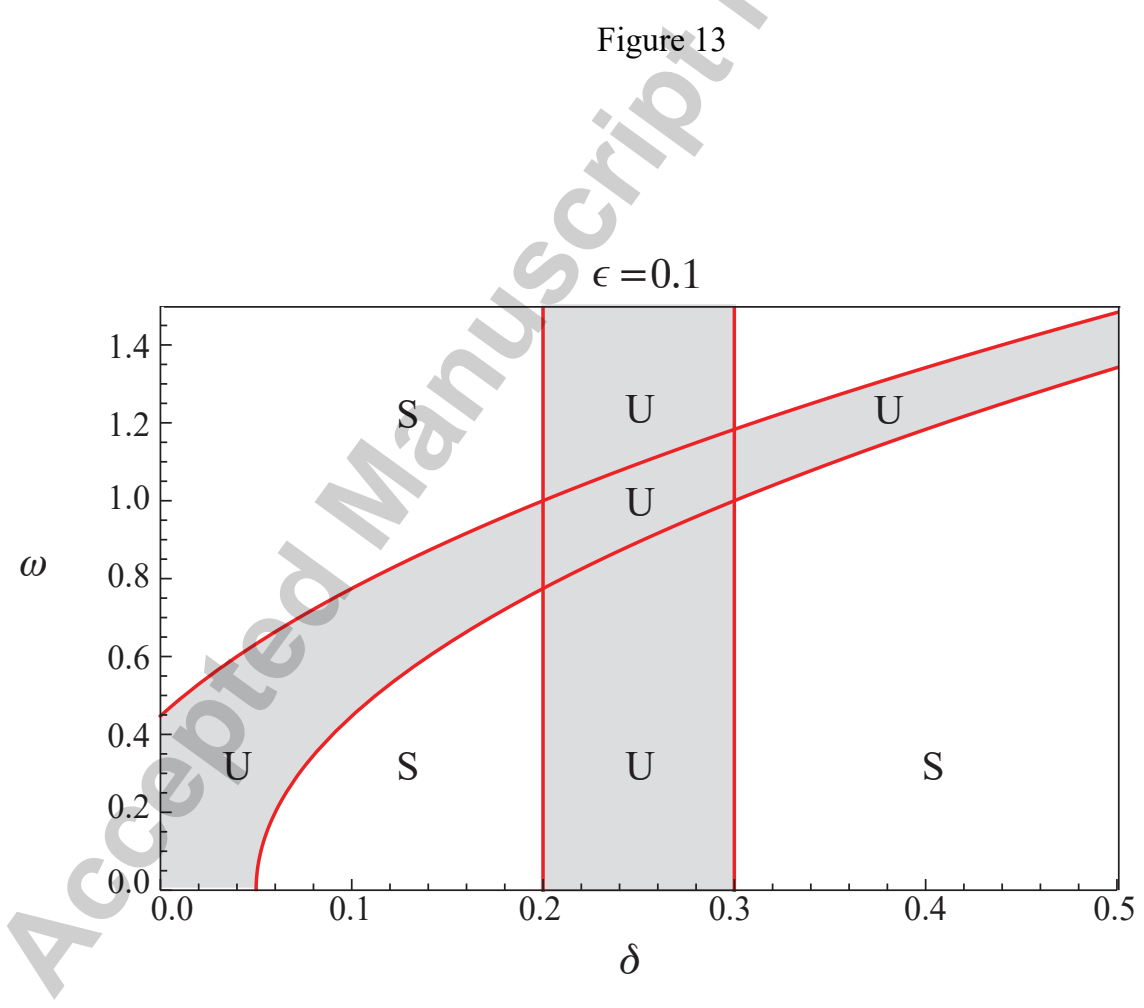


Figure 14

a

${ }_{44} \epsilon=0.1$

$\omega$

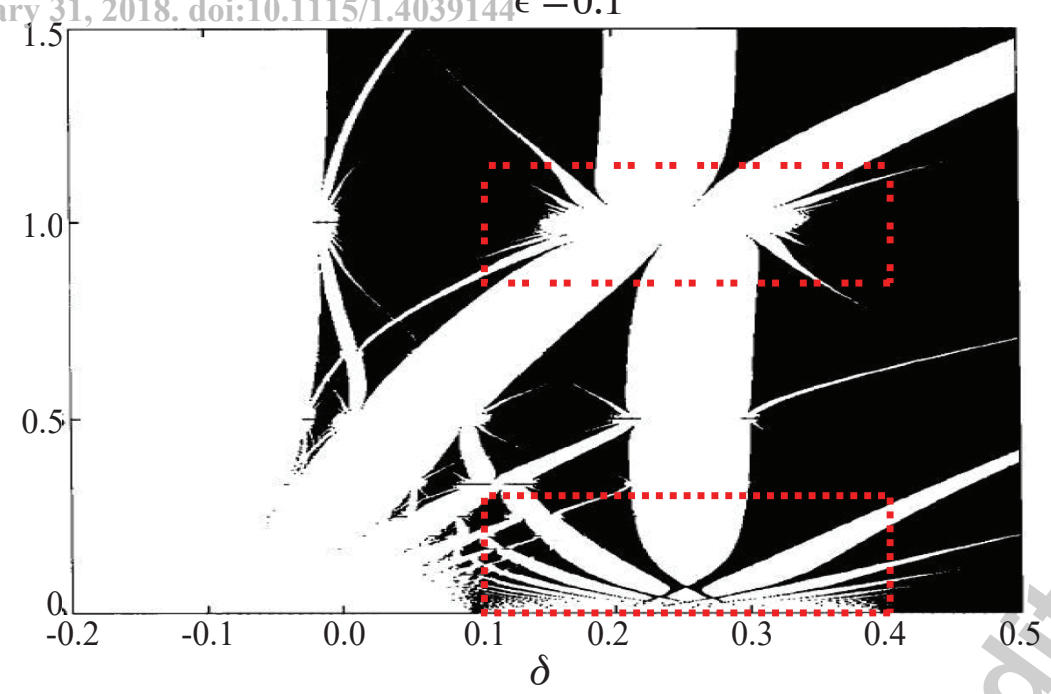

b)

$\omega$

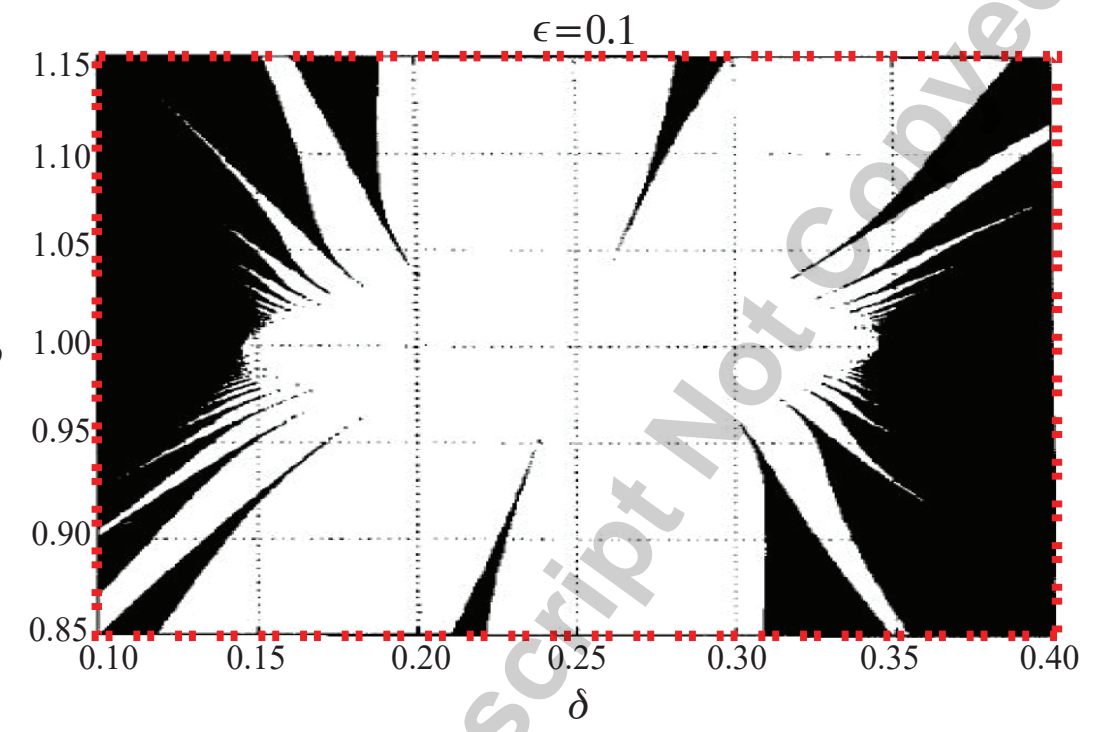

c)

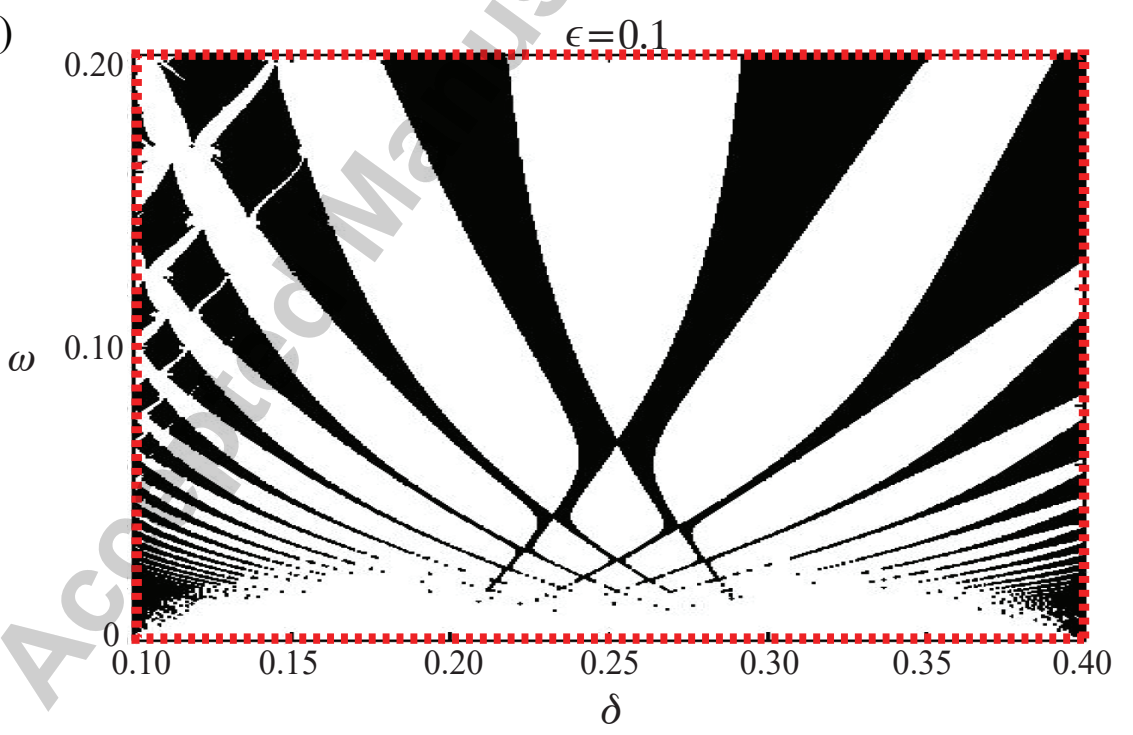



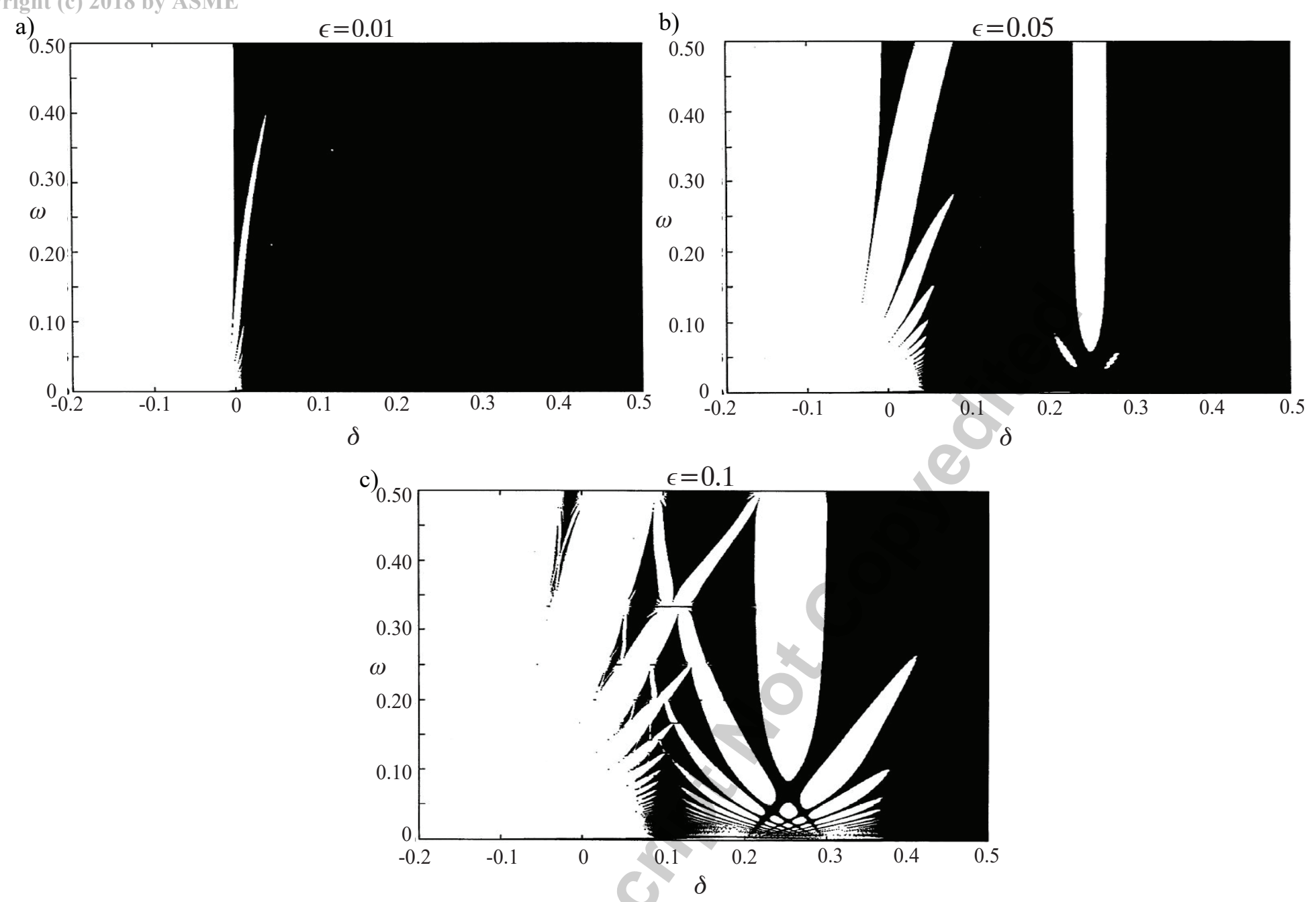

d)

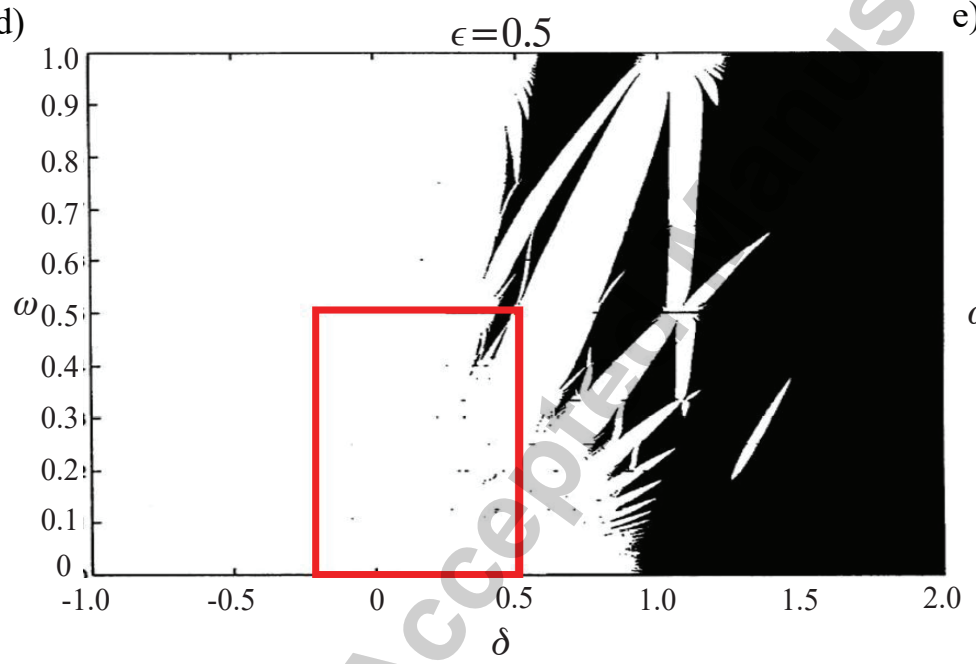

e)

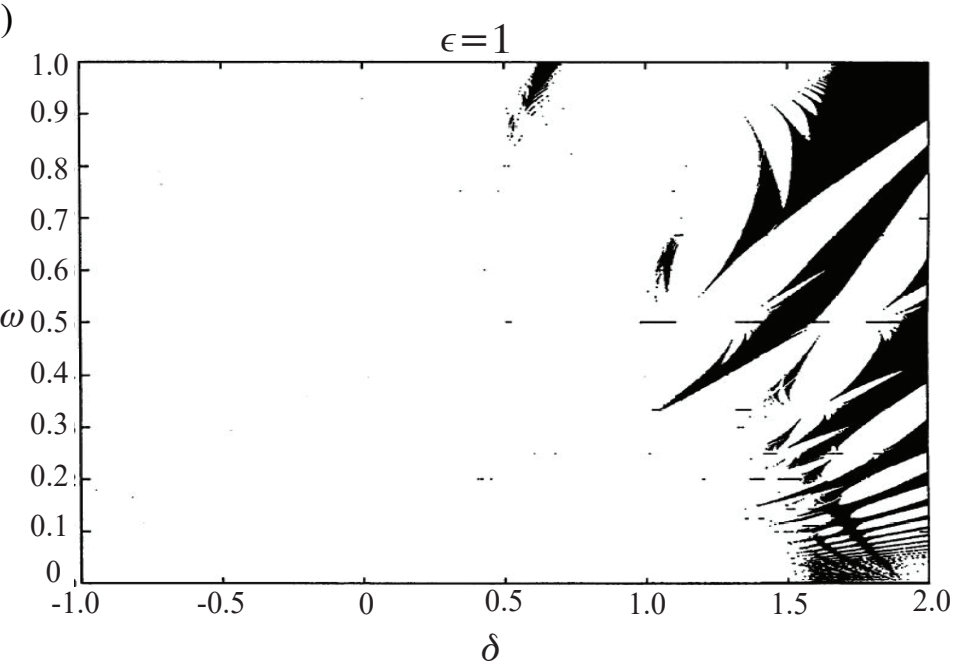


Figure 16

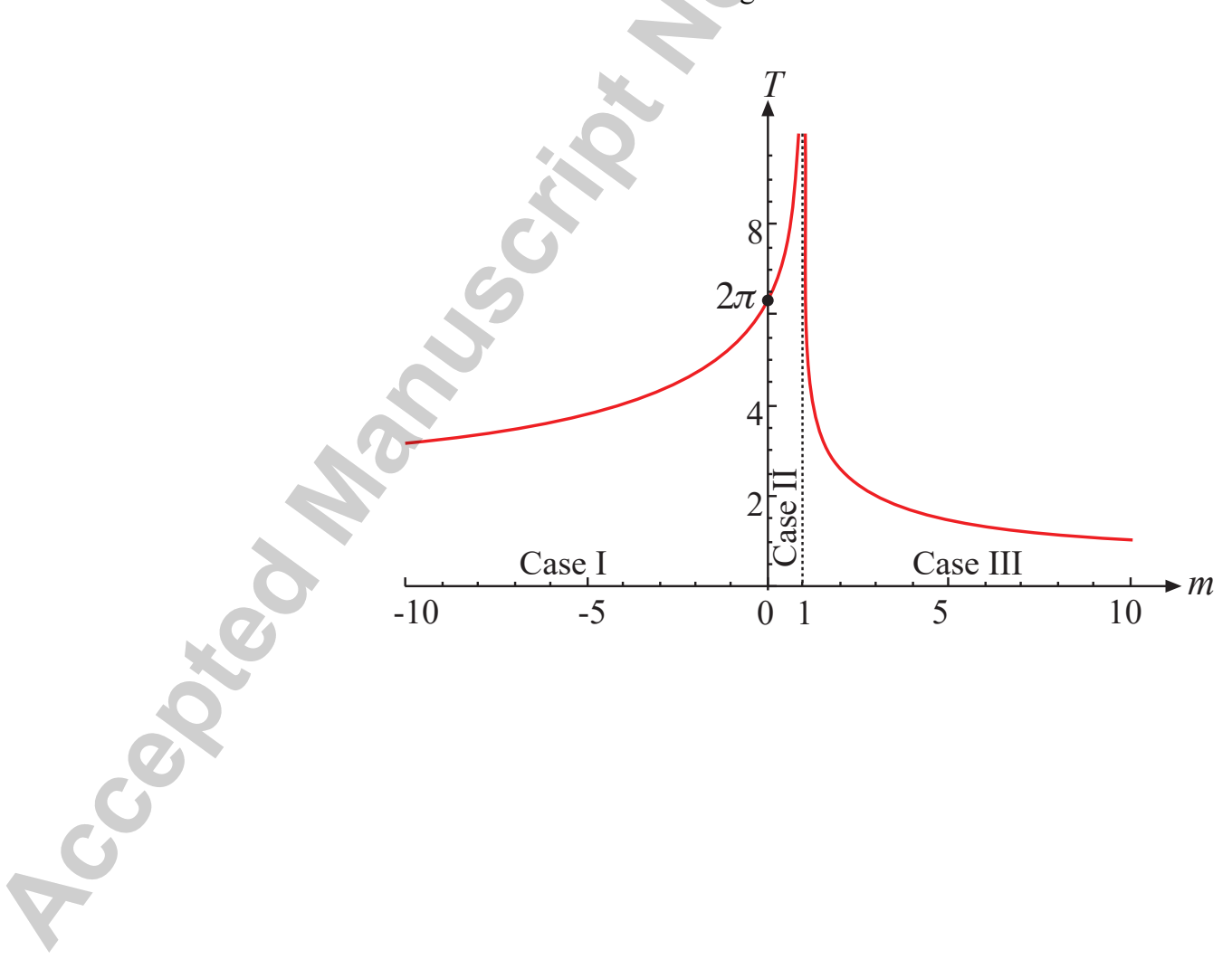


Figure 17

a)

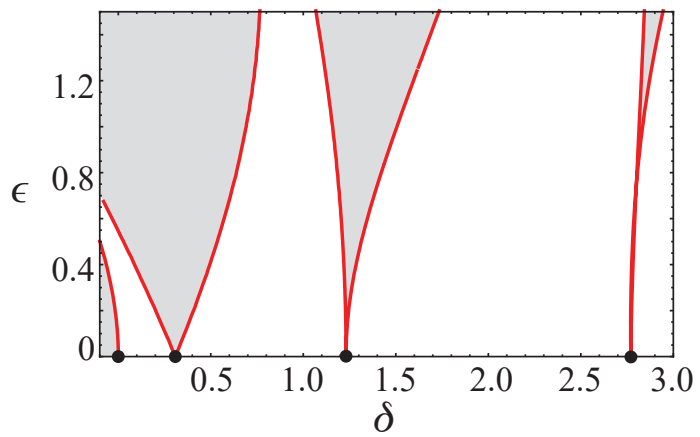

b)

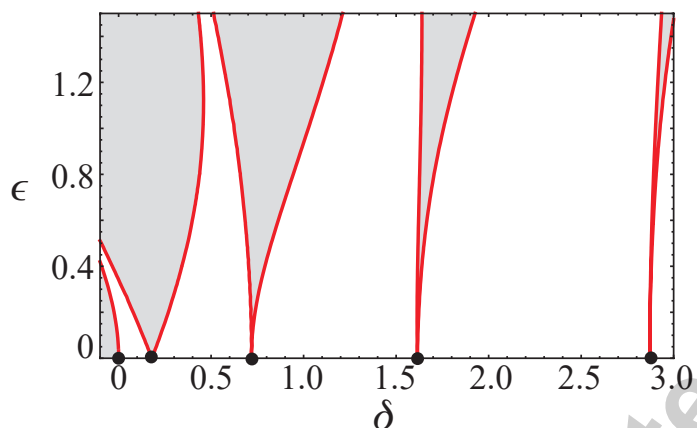

c)

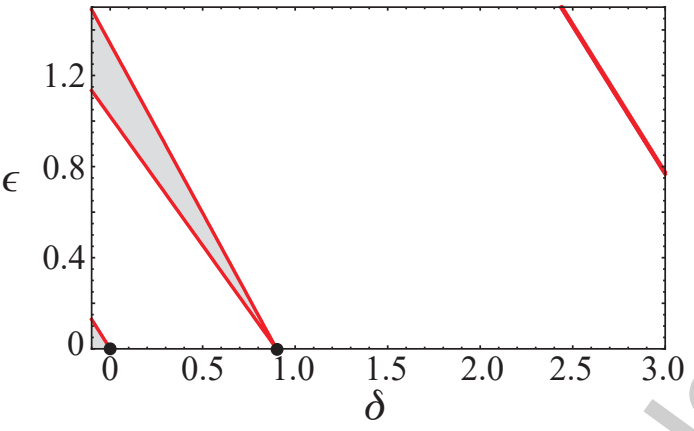


Figure B.1.

Applied Mechanics Reviews. Received August 07, 2017;

Accepted manuscript posted January 31| 2018. doi:10.1115/1.403914.

Copyright (c) 2018 by ASME

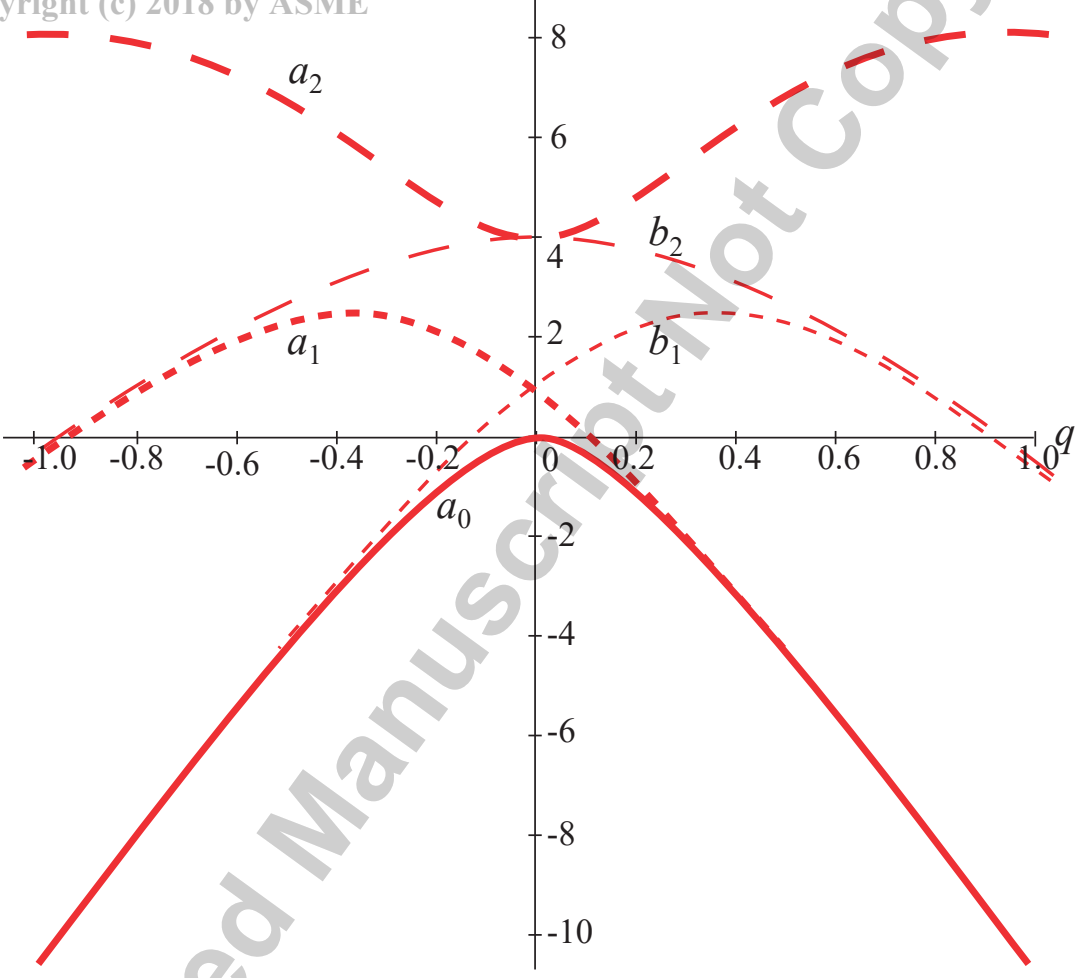

Downloaded From: http://appliedmechanicsreviews.asmedigitalcollection.asme.org/ on 02/13/2018 TerI 
Applied Mechanics Reviews. Received August 07, 2017; Animation 1

Accepted manuscript posted January 31, 2018. doi:10.1115/1.4039144

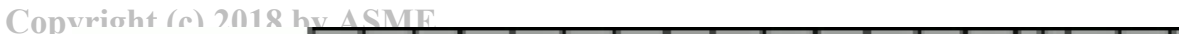

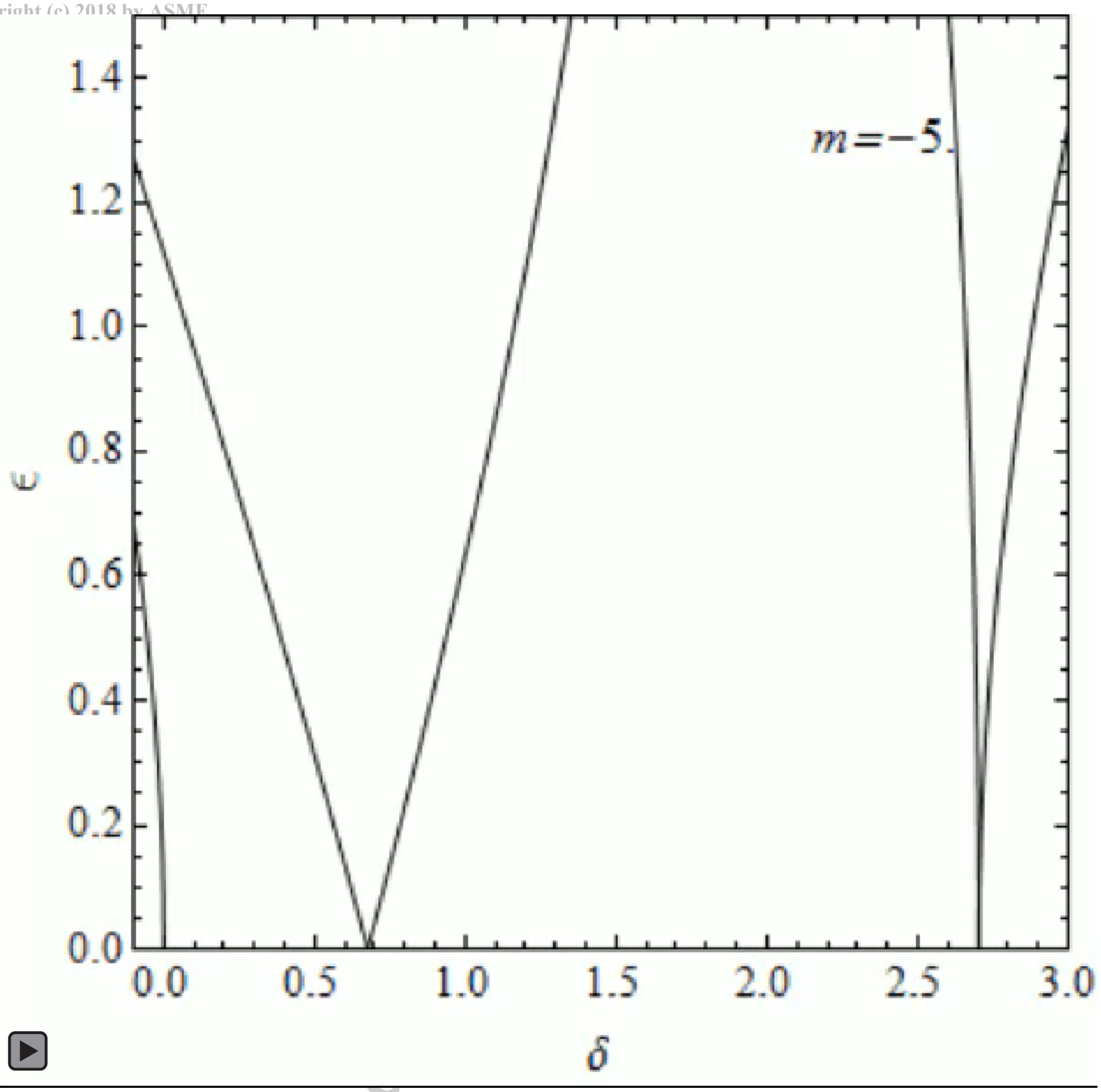

